\title{
GROUP-VALUED IMPLOSION AND PARABOLIC STRUCTURES
}

\author{
JACQUES HURTUBISE, LISA JEFFREY, AND REYER SJAMAAR
}

\begin{abstract}
The purpose of this paper is twofold. First we extend the notion of symplectic implosion to the category of quasi-Hamiltonian K-manifolds, where $\mathrm{K}$ is a simply connected compact Lie group. The imploded cross-section of the double $\mathrm{K} \times \mathrm{K}$ turns out to be universal in a suitable sense. It is a singular space, but some of its strata have a nonsingular closure. This observation leads to interesting new examples of quasi-Hamiltonian K-manifolds, such as the "spinning $2 \mathrm{n}$-sphere" for $\mathrm{K}=\mathbf{S U}(\mathrm{n})$. Secondly we construct a universal ("master") moduli space of parabolic bundles with structure group K over a marked Riemann surface. The master moduli space carries a natural action of a maximal torus of $\mathrm{K}$ and a torus-invariant stratification into manifolds, each of which has a symplectic structure. An essential ingredient in the construction is the universal implosion. Paradoxically, although the universal implosion has no complex structure (it is the four-sphere for $\mathrm{K}=\mathbf{S U}(2)$ ), the master moduli space turns out to be a complex algebraic variety.
\end{abstract}

\section{CONTENTS}

\begin{tabular}{|lr|}
\hline 1. Introduction & 1 \\
\hline 2. Hamiltonian and quasi-Hamiltonian manifolds & 3 \\
\hline 3. Imploded cross-sections & 6 \\
\hline 4. The universal imploded cross-section & 12 \\
\hline 5. Implosion and weighted projective spaces & 21 \\
\hline 6. The master moduli space & 25 \\
\hline Appendix A. The coniugation action & 28 \\
\hline Appendix B. The spinning 2n-sphere & 34 \\
\hline Notation Index & 38 \\
\hline References & 38 \\
\hline
\end{tabular}

\section{INTRODUCTION}

An important first step in understanding a symplectic manifold $M$ equipped with a Hamiltonian action of a compact group $\mathrm{K}$ is a form of abelianization. This involves taking the inverse image under the moment map $\Phi: M \rightarrow \mathfrak{k}^{*}$ of a closed Weyl chamber $\overline{\mathcal{C}}$ in the dual of the Lie algebra of a maximal torus T. According to the Guillemin-Sternberg cross-section theorem the inverse image $\Phi^{-1}(\mathcal{C})$ of the open chamber $\mathcal{C}$ is a smooth symplectic submanifold of $M$ and carries a natural Hamiltonian T-action, which encodes many of the properties of $M$. However, the preimage of the boundary facets of $\mathcal{C}$ is in general neither smooth nor symplectic. One obtains from $\Phi^{-1}(\overline{\mathrm{C}})$ a symplectic variety (a stratified space with symplectic

Date: 27 February 2004.

J. Hurtubise was partially supported by grants from NSERC and FQRNT, L. Jeffrey was partially supported by grants from NSERC, and R. Sjamaar was partially supported by NSF Grant DMS0071625. LJ and RS gratefully acknowledge the hospitality of the Erwin Schrödinger International Institute for Mathematical Physics. 
strata) by "imploding" certain orbits in the boundary pieces. This is the subject of [GJS02. As shown in that paper, the implosion of the cotangent bundle $T^{*} \mathrm{~K}$ plays the role of a universal implosion, from which all other implosions are derived in a simple way.

In parallel to the theory of Hamiltonian K-manifolds, Alekseev, Malkin, and Meinrenken introduced in [AMM98] a notion of quasi-Hamiltonian K-manifolds, which come equipped with moment maps with values in the group K. QuasiHamiltonian K-manifolds and their moment maps share many of the features of the Hamiltonian ones, such as reductions and cross-sections. In this paper, we define the notion of symplectic implosion of quasi-Hamiltonian K-manifolds, where $\mathrm{K}$ is a simply connected compact Lie group. This simultaneously generalizes the results of [GJS02] (where imploded cross-sections were defined) and |HJ00| (where imploded cross-sections of particular quasi-Hamiltonian K-manifolds were defined in the special case $K=\mathbf{S U}(2)$ ). In the course of doing this we define a universal implosion, obtained as the imploded cross-section of the "double" DK $=\mathrm{K} \times \mathrm{K}$.

As an unexpected byproduct of this work we find new examples of quasiHamiltonian K-manifolds, in particular the "spinning 2n-sphere", which generalizes the "spinning four-sphere" described in [HJ00] and [AMW02].

The motivation for developing this theory came from one particularly important example, namely the space of representations of the fundamental group of a punctured Riemann surface (obtained by puncturing a closed Riemann surface at points $p_{1}, p_{2}, \ldots, p_{n}$ ) with values in a simply connected compact $K$. Fixing the conjugacy classes $C=\left(C_{1}, C_{2}, \ldots, C_{n}\right)$ of the image in $K$ of small loops around the punctures, one has symplectic moduli spaces $M(\Sigma, C)$, about which some quite beautiful results are known, in particular the theorem of Mehta and Sehadri [MS80] saying that $M(\Sigma, C)$ is diffeomorphic to a space of parabolic vector bundles over the closed Riemann surface. One would like to have a similar result about the space of all representations. There is no hope, however, of it being symplectic, as in many cases it is not even even-dimensional.

We construct a "master moduli space" $M$ which is in some ways the next best thing. Let $\mathcal{A} \subseteq \mathrm{t}$ be the fundamental alcove of $\mathrm{K}$. The image of $\overline{\mathcal{A}}$ under the exponential map intersects every conjugacy class in $K$ in exactly one point. The space $M$ is a stratified space with symplectic strata equipped with a Hamiltonian $\mathrm{T}^{\mathrm{n}}$-action and moment map $M \rightarrow(\exp \overline{\mathcal{A}})^{n}$. The reduction of $M$ at the $n$-tuple $C$ is then the parabolic moduli space $M(\Sigma, C)$ with conjugacy classes $C$ at the punctures.

It is reasonable to hope that there is a version of the Mehta-Seshadri theorem for the master moduli space $M$, and this is indeed the case: for $K=\mathbf{S U}(n)$ one has a complex moduli space $\mathcal{M}$ of "framed parabolic sheaves", and a homeomorphism of $M$ with $\mathcal{M}$. The parabolic moduli spaces $M(\Sigma, C)$ are GIT quotients of $\mathcal{M}$ under the action of the complexification of the group $\mathrm{T}^{\mathrm{n}}$. The complex algebraic variety $\mathcal{M}$ parametrizes sheaves on the Riemann surface incorporating as extra data a parabolic structure at the punctures and a framing of the successive quotients of the natural flag attached to the parabolic structure. The complexification of $T^{n}$ acts transitively on these framings. A surprising feature is that the construction includes all possible types of flags, ranging from the generic, maximal flag to the minimal, trivial one. The type of the flag for $M(\Sigma, C)$ at $p_{i}$ is determined by the face of $\mathcal{A}$ to which $C_{i}$ belongs. This complex picture is not treated in this paper, but will be the subject of $\mid \mathrm{HJS}$. 
The layout of this paper is as follows. In Section 2 we recall the definition and basic properties of quasi-Hamiltonian K-manifolds. In Section 3 we recall the definition of imploded cross-sections in the Hamiltonian case and extend it to the quasi-Hamiltonian case. In Section 4 we define the "universal implosion" (the implosion of the double DK), after reviewing the corresponding object in the Hamiltonian category (the implosion of $\mathrm{T}^{*} \mathrm{~K}$ ). In Section [5 we describe the universal imploded cross-section as the orbit of a toric variety embedded in a representation of K. In Section $\square$ we descibe the construction of the master moduli space via implosion. In Appendix A we summarize some basic facts concerning the conjugation action of a compact Lie group and in Appendix B we construct the spinning $2 n$-sphere. A notation index can be found at the end of the paper.

\section{HAMILTONIAN AND QUASI-HAMILTONIAN MANIFOLDS}

Group-valued moment maps. Let $\mathrm{K}$ be a compact connected Lie group with Lie algebra $\mathfrak{k}=\mathrm{T}_{1} \mathrm{~K}$. Recall that a Hamiltonian $\mathrm{K}$-manifold is a symplectic manifold $(M, \omega)$ equipped with an action of $K$ which satisfies

$$
\iota\left(\xi_{M}\right) \omega=d\langle\Phi, \xi\rangle
$$

for an $\mathrm{Ad}^{*}$-equivariant map $\Phi: M \rightarrow \mathfrak{k}^{*}$, called the moment map for the action. Here $\xi_{M}$ is the vector field generated by $\xi \in \mathfrak{k}$. Alekseev et al. [AMM98] have extended this notion in the following manner. Recall that the Maurer-Cartan forms $\theta_{\mathrm{L}}, \theta_{\mathrm{R}} \in \Omega^{1}(\mathrm{~K}, \mathfrak{k})$ are defined by $\theta_{\mathrm{L}, \mathrm{g}}\left(\mathrm{L}(\mathrm{g})_{*} \xi\right)=\xi$ and $\theta_{\mathrm{R}, \mathrm{g}}\left(\mathrm{R}(\mathrm{g})_{*} \xi\right)=\xi$ for $\xi \in \mathfrak{k}$. Here $\mathrm{L}(\mathrm{g})$ denotes left multiplication and $\mathrm{R}(\mathrm{g})$ right multiplication by $\mathrm{g}$. Fix a $\mathrm{K}$ invariant inner product $(\cdot, \cdot)$ on $\mathfrak{k}$ and let $\chi=\frac{1}{12}\left(\theta_{L},\left[\theta_{L}, \theta_{L}\right]\right)=\frac{1}{12}\left(\theta_{R},\left[\theta_{R}, \theta_{R}\right]\right)$ be the corresponding bi-invariant three-form on $\mathrm{K}$.

2.2. Definition. A quasi-Hamiltonian (or group-valued Hamiltonian) K-manifold is a smooth $\mathrm{K}$-manifold $\mathrm{M}$ equipped with a K-invariant two-form $\omega$ and an Ad-equivariant map $\Phi: M \rightarrow \mathrm{K}$, called the (group-valued) moment map, satisfying the following properties:

(i) $\mathrm{d} \omega=-\Phi^{*} \chi$;

(ii) $\operatorname{ker} \omega_{x}=\left\{\xi_{M}(x) \mid \xi \in \operatorname{ker}(\operatorname{Ad} \Phi(x)+1)\right\}$ for all $x \in M$;

(iii) $\mathfrak{\imath}\left(\xi_{M}\right) \omega=\frac{1}{2} \Phi^{*}\left(\theta_{L}+\theta_{R}, \xi\right)$ for all $\xi \in \mathfrak{k}$.

If $\mathrm{K}$ is a torus, the two-form is symplectic and $\Phi$ is a moment map in the sense of [McD88]. For nonabelian $\mathrm{K}$ the two-form is usually neither nondegenerate nor closed. (But see Appendix B for a quasi-Hamiltonian $\mathbf{S U}(\mathrm{n})$-structure on $\mathbf{C P}^{\mathrm{n}-1}$ whose two-form is symplectic.) Axiom (ii) replaces the closedness of $\omega$, axiom (ii), often referred to as the minimal degeneracy axiom, replaces the nondegeneracy of $\omega$ and axiom (iii) replaces the moment map condition (2.1).

Group-valued moment maps have rather limited functorial properties. A quasiHamiltonian action of $\mathrm{K}$ usually does not restrict to quasi-Hamiltonian actions of its subgroups. For instance, if a product $\mathrm{K}_{1} \times \mathrm{K}_{2}$ acts in a quasi-Hamiltonian fashion on $(M, \omega)$, neither of the factors acts in a quasi-Hamiltonian fashion (unless the other factor happens to be abelian). However, the following properties are straightforward consequences of the definition.

2.3. Lemma. Let $(\mathrm{M}, \omega, \Phi)$ be a quasi-Hamiltonian $\mathrm{K}$-manifold.

(i) For any $\mathrm{g}$ in the centre of $\mathrm{K}, \mathrm{L}(\mathrm{g}) \circ \Phi=\mathrm{R}(\mathrm{g}) \circ \Phi$ is a moment map for the $\mathrm{K}$-action on $(\mathrm{M}, \omega)$. 
(ii) $(\mathrm{M},-\mathrm{w}, \mathrm{i} \circ \Phi)$ is a quasi-Hamiltonian $\mathrm{K}$-manifold, where $\mathrm{i}: \mathrm{K} \rightarrow \mathrm{K}$ denotes inversion.

(iii) If $\mathrm{K}=\mathrm{K}_{1} \times \mathrm{K}_{2}$ where $\mathrm{K}_{2}$ is a torus, then $\pi_{1} \circ \Phi: \mathrm{M} \rightarrow \mathrm{K}_{1}$ is a moment map for the $\mathrm{K}_{1}$-action on $(\mathrm{M}, \mathrm{\omega})$.

(iv) Let $\pi_{\mathrm{K}}: \tilde{\mathrm{K}} \rightarrow \mathrm{K}$ be a covering homomorphism and let $\pi_{\mathrm{M}}: \tilde{M} \rightarrow \mathrm{M}$ be the induced covering, as in the pullback diagram

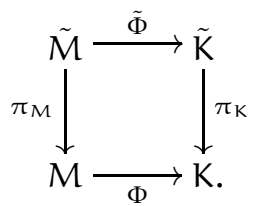

Then there is a unique $\tilde{\mathrm{K}}$-action on $\tilde{\mathrm{M}}$ such that $\tilde{\Phi}$ is equivariant and $\pi_{\mathrm{M}}$ is $\pi_{\mathrm{K}}$-equivariant. Let $\tilde{\omega}=\pi_{M}^{*} \omega$. Then $\tilde{\Phi}$ is a moment map for the $\tilde{\mathrm{K}}$-action on $(\tilde{M}, \tilde{\omega})$.

(v) Let $\mathrm{K}_{1}$ be a closed central subgroup of $\mathrm{K}$ which acts trivially on $\mathrm{M}$. Let $\mathrm{K}_{2}=$ $\mathrm{K} / \mathrm{K}_{1}$ and let $\Phi_{2}: \mathrm{M} \rightarrow \mathrm{K}_{2}$ be the composition of $\Phi$ with the quotient map $\mathrm{K} \rightarrow \mathrm{K}_{2}$. Then the triple $\left(\mathrm{M}, \omega, \Phi_{2}\right)$ is a quasi-Hamiltonian $\mathrm{K}_{2}$-manifold.

The triple $(M,-\omega, i \circ \Phi)$ in (ii) is the quasi-Hamiltonian K-manifold opposite to $M$. We shall frequently denote it by $\mathrm{M}^{-}$.

If the moment map $\Phi$ in (iv) lifts to a map $\Phi^{\prime}: M \rightarrow \tilde{K}$, then $\tilde{M}$ is simply a disjoint union of copies of $M$ and $\Phi^{\prime}$ is the restriction of $\tilde{\Phi}$ to one copy.

Fusion. A sort of functoriality for group-valued moment maps holds for restriction to a diagonal subgroup.

2.4. Theorem (AMM98. Theorem 6.1]). Let $(M, \omega, \Phi)$ be a quasi-Hamiltonian $\mathrm{K} \times$ $\mathrm{K} \times \mathrm{H}$-manifold, with moment map $\Phi=\Phi_{1} \times \Phi_{2} \times \Phi_{3}: \mathrm{M} \rightarrow \mathrm{K} \times \mathrm{K} \times \mathrm{H}$. Let $\mathrm{K} \times \mathrm{H}$ act on $\mathrm{M}$ via the embedding $(\mathrm{k}, \mathrm{h}) \mapsto(\mathrm{k}, \mathrm{k}, \mathrm{h})$. Then $\mathrm{M}$ furnished with the two-form $\omega+\frac{1}{2}\left(\Phi_{1}^{*} \theta_{\mathrm{L}}, \Phi_{2}^{*} \theta_{\mathrm{R}}\right)$ and the moment map $\Phi_{1} \Phi_{2} \times \Phi_{3}: \mathrm{M} \rightarrow \mathrm{K} \times \mathrm{H}$ is a quasi-Hamiltonian $\mathrm{K} \times \mathrm{H}$-manifold.

This restriction process from $\mathrm{K} \times \mathrm{K} \times \mathrm{H}$ to $\mathrm{K} \times \mathrm{H}$ is called internal fusion. The most important class of examples is that of a Cartesian product $M_{1} \times M_{2}$ of a quasiHamiltonian $\mathrm{K}$-manifold $\left(\mathrm{M}_{1}, \omega_{1}, \Phi_{1}\right)$ and a quasi-Hamiltonian $\mathrm{K} \times \mathrm{H}$-manifold $\left(\mathrm{M}_{2}, \omega_{2}, \Phi_{2} \times \Phi_{3}\right)$. This is a quasi-Hamiltonian $\mathrm{K} \times \mathrm{K} \times \mathrm{H}$-manifold in an obvious way, and fusing the two copies of $\mathrm{K}$ gives rise to a quasi-Hamiltonian $\mathrm{K} \times \mathrm{H}$ manifold called the fusion product $M_{1} \circledast M_{2}$.

Exponentiation and linearization. Let $\left(M, \omega_{0}, \Phi_{0}\right)$ be a Hamiltonian $K$-manifold in the ordinary sense. Let us use the isomorphism $\mathfrak{k} \rightarrow \mathfrak{k}^{*}$ given by the inner product to identify $\mathfrak{k}^{*}$ with $\mathfrak{k}$, so that we can regard $\Phi_{0}$ as a map into $\mathfrak{k}$. The process of exponentiation alters the two-form and the moment map (but not the action) on $M$, namely into $\omega=\omega_{0}+\Phi_{0}^{*} \varpi$ and $\Phi=\exp \circ \Phi_{0}$. Here $\varpi \in \Omega^{2}(\mathfrak{k})$ is the Ad Kinvariant primitive of $-\exp ^{*} \chi \in \Omega^{3}(\mathfrak{k})$ given by setting $\exp _{s} \lambda=\exp (\mathrm{s} \lambda)$ and

$$
\varpi=\frac{1}{2} \int_{0}^{1}\left(\exp _{s}^{*} \theta_{R}, \frac{\partial}{\partial s} \exp _{s}^{*} \theta_{R}\right) d s .
$$


A calculation using

$$
\left(\exp ^{*} \theta_{L}\right)_{\lambda}=\frac{1-e^{-\operatorname{ad} \lambda}}{\operatorname{ad} \lambda} \quad \text { and } \quad\left(\exp ^{*} \theta_{R}\right)_{\lambda}=\frac{e^{\operatorname{ad} \lambda}-1}{\operatorname{ad} \lambda}
$$

yields

$$
\varpi_{\lambda}\left(\xi_{1}, \xi_{2}\right)=\int_{0}^{1}\left(\frac{1-\cosh s \operatorname{ad} \lambda}{\operatorname{ad} \lambda} \xi_{1}, \xi_{2}\right) d s=\left(\frac{\operatorname{ad} \lambda-\sinh \operatorname{ad} \lambda}{(\operatorname{ad} \lambda)^{2}} \xi_{1}, \xi_{2}\right)
$$

for $\lambda, \xi_{1}, \xi_{2} \in \mathfrak{k}$. The triple $(M, \omega, \Phi)$ satisfies axioms (i) and (iii) of Definition 2.2 If in addition all points in $\Phi_{0}(M)$ are regular for the exponential map, axiom (ii) is also satisfied and so $(M, \omega, \Phi)$ is a quasi-Hamiltonian K-manifold. The reverse of exponentiation is linearization. Let $(M, \omega, \Phi)$ be a quasi-Hamiltonian $\mathrm{K}$-manifold. Suppose there exists an Ad-invariant open $U$ in $\mathfrak{k}$ such that exp: $U \rightarrow$ $\mathrm{K}$ is a diffeomorphism onto an open subset containing $\Phi(M)$ (with inverse denoted by $\log$ : exp $\mathrm{U} \rightarrow \mathrm{U}$ ). The linearization of $\mathrm{M}$ is the Hamiltonian $\mathrm{K}$-manifold $\left(M, \omega_{0}, \Phi_{0}\right)$, where $\Phi_{0}=\log \circ \Phi$ and $\omega_{0}=\omega-\Phi_{0}^{*} \varpi$. (See AMM98 $\S 3.3$.)

Quasi-symplectic quotients. It is shown in [AMM98, $\S 8$ 8] that the category of quasi-Hamiltonian K-manifolds is equivalent to a subcategory of the category of infinite-dimensional symplectic manifolds with Hamiltonian actions (in the usual sense) of the loop group LK. It should therefore come as no surprise that many constructions in the category of Hamiltonian manifolds have parallels in the quasiHamiltonian world. In particular there are analogues of symplectic reduction and of symplectic implosion. We review quasi-symplectic reduction below; quasisymplectic implosion is the topic of Section 3

Let $(M, \omega, \Phi)$ be a quasi-Hamiltonian K-manifold. Assume that $\mathrm{K}=\mathrm{K}_{1} \times \mathrm{K}_{2}$ where $K_{2}$ is a torus and let $\Phi_{1}: M \rightarrow K_{1}$ and $\Phi_{2}: M \rightarrow K_{2}$ be the components of $\Phi$. Let $g \in K$. Because $\Phi$ is equivariant, the centralizer $\left(K_{1}\right)_{g}$ acts on the fibre $\Phi_{1}^{-1}(\mathrm{~g})$. The quasi-symplectic quotient or quasi-Hamiltonian reduced space at $\mathrm{g}$ is the topological space

$$
\mathrm{M} / / \mathrm{g} \mathrm{K}_{1}=\Phi_{1}^{-1}(\mathrm{~g}) /\left(\mathrm{K}_{1}\right)_{\mathrm{g}} .
$$

The subscript is usually omitted when $g=1$. In good cases this quotient is a symplectic orbifold.

2.7. Theorem (AMM98, Theorem 5.1]). Suppose that $\mathrm{g}$ is a regular value of $\Phi_{1}$. Then the centralizer $\left(\mathrm{K}_{1}\right)_{\mathrm{g}}$ acts locally freely on the submanifold $\Phi_{1}^{-1}(\mathrm{~g})$. The restriction of $\omega$ to $\Phi_{1}^{-1}(\mathrm{~g})$ is closed and $\left(\mathrm{K}_{1}\right)_{\mathrm{g}}$-basic. The form $\omega_{\mathrm{g}}$ on the orbifold $\mathrm{M} / / \mathrm{g}_{1}$ induced by $\omega$ is nondegenerate. The map $\mathrm{M} / / \mathrm{g} \mathrm{K}_{1} \rightarrow \mathrm{K}_{2}$ induced by $\Phi_{2}$ is a moment map for the induced $\mathrm{K}_{2}$-action on $\mathrm{M} / / \mathrm{g} \mathrm{K}_{1}$.

(AMM98 also covers the case where the second factor $K_{2}$ is nonabelian. Then $\mathrm{M} / / \mathrm{g}$ is not symplectic, but a quasi-Hamiltonian $\mathrm{K}_{2}$-orbifold.)

In the singular case the quotient stratifies into symplectic manifolds according to orbit type. Let $\mathrm{H}$ be a subgroup of $\left(\mathrm{K}_{1}\right)_{\mathrm{g}}$. Recall that the stratum of orbit type $\mathrm{H}$ in $\mathrm{M}$ (with respect to the $\left(\mathrm{K}_{1}\right)_{\mathrm{g}}$-action) is the $\left(\mathrm{K}_{1}\right)_{\mathrm{g}}$-invariant submanifold $\mathrm{M}_{(\mathrm{H})}$ consisting of all points $x$ such that the stabilizer $\left(K_{1}\right)_{g} \cap\left(K_{1}\right)_{x}$ is conjugate to $H$. Put $\mathrm{Z}=\Phi_{1}^{-1}(\mathrm{~g})$ and $Z_{(\mathrm{H})}=\mathrm{Z} \cap \mathrm{M}_{(\mathrm{H})}$. Let $\left\{\mathrm{Z}_{\mathrm{i}} \mid \mathrm{i} \in \mathrm{I}\right\}$ be the collection of connected components of all subspaces $Z_{(H)}$, where $(H)$ ranges over all conjugacy classes of 
subgroups of $\left(\mathrm{K}_{1}\right)_{\mathrm{g}}$. Set-theoretically the quotient is a disjoint union,

$$
M / / g K_{1}=\coprod_{i \in I} Z_{i} /\left(K_{1}\right)_{g} \text {. }
$$

2.9. Theorem. Let $g \in K_{1}$ be arbitrary. Each of the subsets $Z_{i}$ is a submanifold of $M$ and the restriction of $\omega$ to $Z_{i}$ is closed and $\left(K_{1}\right)_{g}$-basic. The orbit space $Z_{i} /\left(K_{1}\right)_{g}$ is a manifold and the form induced by $\omega$ on $\mathrm{Z}_{i} /\left(\mathrm{K}_{1}\right)_{g}$ is nondegenerate. The decomposition (2.8) is a locally normally trivial stratification of $\mathrm{M} / / \mathrm{g} \mathrm{K}_{1}$. The stratification is $\mathrm{K}_{2}$-invariant and the continuous map $\bar{\Phi}_{2}: \mathrm{M} / / \mathrm{g} \mathrm{K}_{1} \rightarrow \mathrm{K}_{2}$ induced by $\Phi_{2}$ restricts to a moment map for the $\mathrm{K}_{2}$-action on each stratum.

Proof. By the quasi-Hamiltonian shifting trick (see [AMM98 Remark 6.2]) we may assume that $g=1$. Choose an invariant open neighbourhood $U$ of $0 \in \mathfrak{k}$ such that exp is a diffeomorphism from $\mathrm{U}$ onto its image and let log: exp $\mathrm{U} \rightarrow \mathrm{U}$ be the inverse. Replace $M$ with the (not necessarily connected) open subset $\Phi^{-1}(\exp U)$. Clearly this does not affect the quotient $M / / K$. Let $\left(M, \omega_{0}, \Phi_{0}\right)$ be the linearization of $(M, \omega, \Phi)$. Observe that $\Phi^{-1}(1)=\Phi_{0}^{-1}(0)$. Hence $\omega=\omega_{0}$ on every submanifold of $M$ contained in $\Phi^{-1}(1)$. The theorem follows therefore from the Hamiltonian case, where we appeal to [SL91, Theorem 2.1].

(Presumably this result too generalizes to nonabelian $\mathrm{K}_{2}$. However, because the linearization $\left(\mathrm{M}, \omega_{0}, \Phi_{0}\right)$ is not a Hamiltonian K-manifold in that case, our proof is valid only for abelian $\mathrm{K}_{2}$.) We call the space $\mathrm{M} / / \mathrm{K}_{1}$ a stratified Hamiltonian $\mathrm{K}_{2^{-}}$ space and refer to the map $\bar{\Phi}_{2}$ as the moment map for the $K_{2}$-action.

\section{IMPLODED CROSS-SECTIONS}

The Hamiltonian case. The first goal of this paper is to develop a quasi-Hamiltonian analogue of symplectic implosion. We start by reviewing this notion in the Hamiltonian case, referring to [GJS02 $\S 2$ ] for details. Symplectic implosion is an "abelianization functor", a crude operation that transmutes a Hamiltonian Kmanifold into a Hamiltonian $\mathrm{T}$-space (where $\mathrm{T}$ is a maximal torus of $\mathrm{K}$ ) retaining some of the relevant features of the original manifold, but at the expense of producing singularities. However, the singular set breaks up into smooth symplectic manifolds in a nice way.

Fix a maximal torus $T$ of $K$ and an open chamber $\mathcal{C}$ in $\mathfrak{t}^{*}$, the dual of $\mathfrak{t}=$ Lie T. The closed chamber $\bar{\complement}$ is a polyhedral cone, which is the disjoint union of $2^{r}$ relatively open faces, where $r$ is the rank of the commutator subgroup $[K, K]$. We define a partial order on the faces by putting $\sigma \leq \tau$ if $\sigma \subseteq \bar{\tau}$.

Let $(M, \omega, \Phi)$ be a connected Hamiltonian K-manifold. The principal face $\sigma_{\text {prin }}$ is the smallest face $\sigma$ of $\mathcal{C}$ such that the Kirwan polytope $\Phi(M) \cap \overline{\mathcal{C}}$ is contained in the closure of $\sigma$. In many cases $\sigma_{\text {prin }}=\mathcal{C}$. The cross-section of $M$ is $\Phi^{-1}\left(\sigma_{\text {prin }}\right)$. This is a T-invariant connected symplectic submanifold of $M$. The torus action on the cross-section is Hamiltonian with moment map equal to the restriction of the $\mathrm{K}$-moment map, and the $\mathrm{K}$-invariant subset $\mathrm{K}^{-1}\left(\sigma_{\text {prin }}\right)$ is open and dense in $\mathrm{M}$.

The imploded cross-section is a "completion" of the cross-section to a stratified space with symplectic strata. It is obtained by taking the preimage of the closed chamber, $\Phi^{-1}(\overline{\mathcal{C}})$, which stratifies into smooth manifolds in a natural way, and by quotienting out the null-foliation of the form $\omega$ on each stratum. To wit, declare two points $m_{1}$ and $m_{2}$ in $\Phi^{-1}(\overline{\mathcal{C}})$ to be equivalent if there exists $k$ in the commutator 
group $\left[K_{\Phi\left(m_{1}\right)}, K_{\Phi\left(m_{1}\right)}\right]$ such that $m_{2}=k m_{1}$. (Here $K_{\xi}$ denotes the centralizer of $\xi \in \mathfrak{k}^{*}$.) Then the imploded cross-section is the quotient space

$$
\mathrm{M}_{\text {impl }}=\Phi^{-1}(\overline{\mathrm{C}}) / \sim,
$$

equipped with the quotient topology. Set-theoretically it is a disjoint union

$$
M_{\text {impl }}=\coprod_{\sigma \leq \mathcal{C}} \Phi^{-1}(\sigma) /\left[K_{\sigma}, K_{\sigma}\right]
$$

over the faces $\sigma$ of $\mathcal{C}$. Here $K_{\sigma}$ is the centralizer of (any point in) the face $\sigma$. The pieces in the decomposition (3.1) are usually not manifolds. However, the decomposition can be refined into a stratification of $M_{\text {impl }}$ with symplectic strata. There is a unique open stratum, which is dense in $M_{\mathrm{impl}}$ and symplectomorphic to the cross-section $\Phi^{-1}\left(\sigma_{\text {prin }}\right)$. (See GJS02 Theorems 2.10 and 5.10].) These facts are most easily established by using slices for the coadjoint action on $\mathfrak{k}^{*}$. The natural slice at a face $\sigma$ is the subset of $\mathfrak{k}_{\sigma}^{*}$ given by

$$
\mathfrak{S}_{\sigma}=\operatorname{Ad}^{*}\left(K_{\sigma}\right) \operatorname{star} \sigma
$$

where star $\sigma$ denotes the open star $\bigcup_{\tau>\sigma} \tau$ of $\sigma$. $\left(\mathfrak{S}_{\sigma}\right.$ is a slice for the coadjoint action in the sense that $\mathrm{KS}_{\sigma}$ is open in $\mathfrak{k}_{\sigma}^{*}$ and $\mathrm{K}$-equivariantly diffeomorphic to the associated bundle $\mathrm{K} \times{ }^{\mathrm{K}_{\sigma}} \mathfrak{S}_{\sigma}$. Note that $\mathfrak{S}_{\sigma}=\sigma$ if $\sigma=\mathcal{C}$.) The set $M_{\sigma}=\Phi^{-1}\left(\mathfrak{S}_{\sigma}\right)$ is a $\mathrm{K}_{\sigma}$-invariant connected symplectic submanifold of $M$ and $\Phi^{-1}(\sigma) /\left[\mathrm{K}_{\sigma}, \mathrm{K}_{\sigma}\right]$ is the symplectic quotient of $M_{\sigma}$ with respect to the action of $\left[K_{\sigma}, K_{\sigma}\right]$. Thence, by appealing to [SL91. Theorem 2.1] we obtain a refinement of the decomposition 3.1 into symplectic strata.

The imploded moment map is the continuous map $\Phi_{\text {impl }}: M_{\text {impl }} \rightarrow t^{*}$ induced by $\Phi$. The imploded cross-section is an abelianization of $M$ in the sense that it carries a natural T-action which preserves the stratification. The restriction of $\Phi_{\text {impl }}$ to each stratum is a moment map for the T-action; the image of $\Phi_{\text {impl }}$ is equal to $\Phi(M) \cap \overline{\mathrm{e}}$, the Kirwan polytope of $M$; and the symplectic quotients of $M_{\text {impl with }}$ respect to the T-action are identical to the symplectic quotients of $M$ with respect to the K-action. (See [GJS02, Theorem 3.4].)

The quasi-Hamiltonian case. Now let $(M, \omega, \Phi)$ be a connected quasi-Hamiltonian K-manifold. Proceeding by analogy with the Hamiltonian case we shall define the imploded cross-section of $M$ and prove that it stratifies into quasi-Hamiltonian T-manifolds. To obtain good results we shall assume for the remainder of the paper that $\mathrm{K}$ is simply connected.

Let $\mathcal{C}^{\vee}$ be the chamber in $\mathfrak{t}$ dual to $\mathcal{C}$ and let $\mathcal{A}$ be the unique (open) alcove contained in $\mathcal{C}^{\vee}$ such that $0 \in \overline{\mathcal{A}}$. The exponential map induces a homeomorphism from $\overline{\mathcal{A}} / \pi_{1}(\mathrm{~K})=\overline{\mathcal{A}}$ to $\mathrm{T} / \mathrm{W} \cong \mathrm{K} / \mathrm{Ad} \mathrm{K}$, the space of conjugacy classes in $\mathrm{K}$. (Cf. [Bou82 Ch. 9, § 5.2, Corollaire 1].) Here $W=N(T) / T$ denotes the Weyl group of $(\mathrm{K}, \mathrm{T})$. In particular the restriction of the exponential map to $\overline{\mathcal{A}}$ is injective. For $\mathrm{g}$ in $\mathrm{K}$ let $\mathrm{K}_{\mathrm{g}}=\{\mathrm{h} \in \mathrm{K} \mid \mathrm{hg}=\mathrm{gh}\}$ denote the centralizer of $\mathrm{g}$. For points $\mathrm{m}_{1}$, $\mathrm{m}_{2} \in \Phi^{-1}(\exp \overline{\mathcal{A}})$ define $\mathrm{m}_{1} \sim \mathrm{m}_{2}$ if $\mathrm{m}_{2}=\mathrm{km}_{1}$ for some $\mathrm{k} \in\left[\mathrm{K}_{\Phi\left(\mathrm{m}_{1}\right)}, \mathrm{K}_{\Phi\left(\mathrm{m}_{1}\right)}\right]$. Since $\Phi$ is Ad-equivariant, $\mathrm{m}_{1} \sim \mathrm{m}_{2}$ implies $\Phi\left(\mathrm{m}_{1}\right)=\Phi\left(\mathrm{m}_{2}\right)$, and hence $\sim$ is an equivalence relation.

3.2. Definition. The imploded cross-section of $M$ is the quotient space $M_{\text {impl }}=$ $\Phi^{-1}(\exp \overline{\mathcal{A}}) / \sim$, equipped with the quotient topology. The imploded moment map $\Phi_{\text {impl }}$ is the continuous map $M_{\text {impl }} \rightarrow \mathrm{T}$ induced by $\Phi$. 
Imitating GJS02, Lemma 2.3] one proves easily that the quotient map from $\Phi^{-1}(\exp \overline{\mathcal{A}})$ to $M_{\text {impl }}$ is proper and that $M_{\text {impl }}$ is Hausdorff, locally compact and second countable. The action of T preserves $\Phi^{-1}(\exp \overline{\mathcal{A}})$ and descends to a continuous action on $M_{\text {impl }}$. The image of the imploded moment map is $\Phi(M) \cap \exp \overline{\mathcal{A}}$, the moment polytope of $M$.

The imploded cross-section is seldom a manifold, but we shall see that it partitions into manifolds, each of which carries a symplectic structure. The following discussion draws on some standard facts about the conjugation action reviewed in Appendix $\mathrm{A}$ Lemma A.1 shows that we have an analogue of the decomposition (3.1) into orbit spaces,

$$
M_{\text {impl }}=\coprod_{\sigma \leq \mathcal{A}} \Phi^{-1}(\exp \sigma) /\left[K_{\sigma}, K_{\sigma}\right],
$$

where this time $\sigma$ ranges over the faces of the alcove $\mathcal{A}$ and $\mathrm{K}_{\sigma}$ stands for the centralizer of $\exp \sigma$. Let us write $X_{\sigma}=\Phi^{-1}(\exp \sigma) /\left[K_{\sigma}, K_{\sigma}\right]$. Each of the $X_{\sigma}$ is invariant under the T-action and the imploded moment map carries $X_{\sigma}$ into $\exp \sigma$.

Fix a face $\sigma \leq \mathcal{A}$ and consider its open star star $\sigma=\bigcup_{\tau \geq \sigma} \tau$. By Proposition A.11 the set $\mathfrak{S}_{\sigma}=\operatorname{Ad}\left(K_{\sigma}\right) \exp (\operatorname{star} \sigma) \subseteq K_{\sigma}$ is a slice for the conjugation action of $\mathrm{K}$ on itself. Equivariant maps are transverse to slices, so the $K_{\sigma}$-invariant subset $M_{\sigma}=\Phi^{-1}\left(\mathfrak{S}_{\sigma}\right)$ is a submanifold of $M$, called the cross-section of $M$ over $\sigma$. As in the Hamiltonian case, the principal face $\sigma_{\text {prin }}$ for $M$ is defined as the smallest $\sigma \leq \mathcal{A}$ such that the moment polytope $\Phi(M) \cap \overline{\mathcal{A}}$ is contained in the closure of $\sigma$. The principal cross-section is $M_{\sigma_{\text {prin }}}$.

3.4. Theorem (quasi-Hamiltonian cross-sections). Let $\sigma$ be a face of $\mathcal{A}$.

(i) $M_{\sigma}$ is a connected quasi-Hamiltonian $\mathrm{K}_{\sigma}$-manifold with moment map $\Phi_{\sigma}=$ $\left.\Phi\right|_{M_{\sigma}}$.

(ii) The action $\mathrm{K} \times \mathrm{M}_{\sigma} \rightarrow \mathrm{M}$ induces a diffeomorphism $\mathrm{K} \times \mathrm{K}_{\sigma} \mathrm{M}_{\sigma} \rightarrow \mathrm{K} \mathrm{M}_{\sigma}$. If $\mathrm{M}_{\sigma}$ is nonempty, then $\mathrm{KM}_{\sigma}$ is dense in $\mathrm{M}$.

(iii) The commutator subgroup of $\mathrm{K}_{\sigma_{\text {prin }}}$ acts trivially on the principal cross-section $M_{\sigma_{\text {prin }}}$. Hence $M_{\sigma_{\text {prin }}}$ is symplectic.

Proof. See [AMM98, $\S 7$ for a proof that $M_{\sigma}$ is a quasi-Hamiltonian $K_{\sigma}$-manifold.

The diffeomorphism $K \times{ }^{K_{\sigma}} M_{\sigma} \cong K M_{\sigma}$ follows from the slice theorem, Proposition A.11 The connectedness of $M_{\sigma}$ and denseness of $K M_{\sigma}$ are proved as in the Hamiltonian case, [LMTW98, §3], using the simple connectivity of the homogeneous space $\mathrm{K} / \mathrm{K}_{\sigma}$.

Put $\sigma=\sigma_{\text {prin }}$. Then $\Phi(M) \cap \exp \overline{\mathcal{A}} \subseteq \bar{\sigma}$, so $M_{\sigma}=\Phi^{-1}(\exp \sigma)$. In particular, $\mathrm{K}_{\Phi(\mathrm{x})}=\mathrm{K}_{\sigma}$ for all $x \in M_{\sigma}$. Now fix $x \in M_{\sigma}$ and $v \in \mathrm{T}_{x} M_{\sigma}$. Select a curve $x_{t}$ in $M_{\sigma}$ with $x_{0}=x$ and $x_{0}^{\prime}=v$. Let $\xi \in \mathfrak{k}_{\sigma}$. Since $\mathfrak{k}_{\sigma}=\operatorname{ker}\left(\operatorname{Ad}\left(\Phi\left(x_{t}\right)\right)-1\right)$ we have $\operatorname{Ad}\left(\Phi\left(x_{t}\right)\right) \xi=\xi$ for all $t$. Differentiating this identity and setting $t=0$ yields

$$
\left[\xi,\left(\Phi_{\sigma}^{*} \theta_{\mathrm{L}, \sigma}\right)_{x}(v)\right]=\left[\xi,\left(\Phi^{*} \theta_{\mathrm{L}}\right)_{x}(v)\right]=\left[\xi, \mathrm{L}\left(\Phi(x)^{-1}\right)_{*} \mathrm{~T}_{\chi} \Phi(v)\right]=0 .
$$

Here $\theta_{\mathrm{L}, \sigma}$ denotes the Maurer-Cartan form on $K_{\sigma}$. This means that the image of $\left(\Phi_{\sigma}^{*} \theta_{L, \sigma}\right)_{\chi}: T_{\chi} M_{\sigma} \rightarrow \mathfrak{k}_{\sigma}$ is contained in the centre of $\mathfrak{k}_{\sigma}$. According to AMM98. Proposition 4.1.3] this image is equal to the orthogonal complement $\mathfrak{k}_{x}^{\perp}$ of $\mathfrak{k}_{x}$ in $\mathfrak{k}_{\sigma}$. In other words, $\mathfrak{k}_{x}^{\perp} \subseteq \mathfrak{z}\left(\mathfrak{k}_{\sigma}\right)$, which implies $\left[\mathfrak{k}_{\sigma}, \mathfrak{k}_{\sigma}\right] \subseteq \mathfrak{k}_{\mathrm{x}}$. Therefore $\left[\mathrm{K}_{\sigma}, \mathrm{K}_{\sigma}\right]$ acts trivially on $M_{\sigma}$. This shows that $M_{\sigma}$ is a quasi-Hamiltonian manifold for the torus $\mathrm{K}_{\sigma} /\left[\mathrm{K}_{\sigma}, \mathrm{K}_{\sigma}\right]$ and in particular is symplectic. 
3.5. Remark. Suppose $\mathrm{K}$ is a product $\mathrm{K}_{1} \times \mathrm{K}_{2}$ and $\mathrm{M}$ is quasi-Hamiltonian with moment map $\Phi=\Phi_{1} \times \Phi_{2}$. Let $\mathcal{A}=\mathcal{A}_{1} \times \mathcal{A}_{2}$ be the alcove of $\mathrm{K}$ and let $\sigma$ be a face of $\mathcal{A}_{2}$. Let $\mathfrak{S}_{\sigma}$ be the corresponding slice in $K_{2}$, and let $M_{\sigma}=\Phi_{2}^{-1}\left(\mathfrak{S}_{\sigma}\right)$. Then $M_{\sigma}$ is a connected quasi-Hamiltonian $K_{1} \times\left(K_{2}\right)_{\sigma}$-manifold with moment map $\Phi_{\sigma}=\left.\Phi\right|_{M_{\sigma}}$. This is proved by noting that $K_{1} \times \mathfrak{S}_{\sigma}$ is a slice for the adjoint action of $K$, that $M_{\sigma}=\Phi^{-1}\left(K_{1} \times \mathfrak{S}_{\sigma}\right)$, and by subsequently applying Theorem 3.4 i).

As in [GJS02. Corollary 2.6] it follows from Theorem 3.4 that the imploded crosssection contains a dense copy of the principal cross-section. We can thus view $M_{\text {impl }}$ as a "completion" of the symplectic manifold $M_{\sigma_{\text {prin }}}$.

3.6. Corollary. The restriction of the quotient map $\Phi^{-1}(\exp \overline{\mathcal{A}}) \rightarrow M_{\text {impl }}$ to the principal cross-section is a homeomorphism onto its image. The image is open and dense in $\mathrm{M}_{\mathrm{impl}}$. Hence $\mathrm{M}_{\mathrm{impl}}$ is connected.

We are now going to show that each of the pieces $X_{\sigma}=\Phi^{-1}(\exp \sigma) /\left[K_{\sigma}, K_{\sigma}\right]$ of the imploded cross-section can be written as a quasi-symplectic quotient (up to a finite covering map). Let $Z\left(K_{\sigma}\right)$ be the centre of $K_{\sigma}$ and $Z\left(K_{\sigma}\right)^{0}$ its unit component. Let $H_{\sigma}$ be the affine subspace of $\mathfrak{t}$ spanned by $\sigma$ and choose $g_{\sigma} \in\left[K_{\sigma}, K_{\sigma}\right] \cap Z\left(K_{\sigma}\right)$ such that $\exp H_{\sigma} \subseteq g_{\sigma} Z\left(K_{\sigma}\right)^{0}$, as in Lemma A.3 iv. By Lemma 2.3 i] $\Phi_{\sigma}^{\prime}=$ $\mathrm{L}\left(\mathrm{g}_{\sigma}^{-1}\right) \circ \Phi_{\sigma}$ is a moment map for the $K_{\sigma}$-action on $M_{\sigma}$. The reason for shifting the moment map is that it enables us to write $\Phi^{-1}(\exp \sigma)$ as the inverse image of the torus $\mathrm{Z}\left(\mathrm{K}_{\sigma}\right)^{0}$.

3.7. Lemma. $\quad$ (i) $\exp \sigma=\mathfrak{S}_{\sigma} \cap g_{\sigma} Z\left(K_{\sigma}\right)^{0}=\mathfrak{S}_{\sigma} \cap Z\left(K_{\sigma}\right)$.

(ii) $\Phi^{-1}(\exp \sigma)=\left(\Phi_{\sigma}^{\prime}\right)^{-1}\left(Z\left(K_{\sigma}\right)^{0}\right)$.

Proof. The inclusion exp $\sigma \subseteq \mathfrak{S}_{\sigma} \cap g_{\sigma} Z\left(K_{\sigma}\right)^{0}$ follows from the choice of $g_{\sigma}$ and the inclusion $\mathfrak{S}_{\sigma} \cap g_{\sigma} Z\left(K_{\sigma}\right)^{0} \subseteq \mathfrak{S}_{\sigma} \cap Z\left(K_{\sigma}\right)$ is obvious. Now let $g \in \mathfrak{S}_{\sigma} \cap Z\left(K_{\sigma}\right)$ and write $g=\operatorname{Ad}(k) \exp \xi$ with $k \in K_{\sigma}$ and $\xi \in \operatorname{star} \sigma$. The assumption $\operatorname{Ad}(k) \exp \xi \in$ $Z\left(K_{\sigma}\right)$ implies $\operatorname{Ad}(k) \exp \xi \in T$, so there exists $w$ in the Weyl group $N_{K_{\sigma}}(T) / T$ such that $\operatorname{Ad}(k) \exp \xi=w \exp \xi$. Using $A .17$ and the fact that $w$ preserves $R_{\sigma}$, the root system of $K_{\sigma}$, we see that $w \exp \xi \in Z\left(K_{\sigma}\right)$ implies $\alpha(\xi) \in 2 \pi i Z$ for all $\alpha \in R_{\sigma,+}$. Since $\xi \in \operatorname{star} \sigma$ we obtain $\alpha(\xi)=\alpha(\sigma)$ from (A.13), and therefore $\xi \in \sigma$ by A.12. Hence $\operatorname{Ad}(k) \exp \xi \in \exp \sigma$, i.e. $\mathfrak{S}_{\sigma} \cap Z\left(K_{\sigma}\right) \subseteq \exp \sigma$.

Let $x \in M_{\sigma}$. Using (i) we see that $\Phi_{\sigma}^{\prime}(x) \in Z\left(K_{\sigma}\right)^{0}$ if and only if $\Phi_{\sigma}(x)=$ $g_{\sigma} \Phi_{\sigma}^{\prime}(x) \in \exp \sigma$.

Let us write $\mathrm{K}_{1}=\left[\mathrm{K}_{\sigma}, \mathrm{K}_{\sigma}\right]$ and $\mathrm{K}_{2}=\mathrm{Z}\left(\mathrm{K}_{\sigma}\right)^{0}$. Recalling that $\mathrm{K}_{\sigma}=\mathrm{K}_{1} \mathrm{~K}_{2}$ we define the finite covering

$$
\tilde{\mathrm{K}}_{\sigma}=\mathrm{K}_{1} \times \mathrm{K}_{2} .
$$

The covering map $\tilde{K}_{\sigma} \rightarrow K_{\sigma}$ is given by multiplication and the covering group is the group $\Gamma_{\sigma}=\mathrm{K}_{1} \cap \mathrm{K}_{2}$ of Lemma A.3 iv, embedded into $\tilde{K}_{\sigma}$ by the antidiagonal map $\mathrm{g} \mapsto\left(\mathrm{g}, \mathrm{g}^{-1}\right)$. Pulling back this covering via the shifted moment map $\Phi_{\sigma}^{\prime}: M_{\sigma} \rightarrow K_{\sigma}$ yields a normal covering map $\pi_{\sigma}: \tilde{M}_{\sigma} \rightarrow M_{\sigma}$ with the same covering group $\Gamma_{\sigma}$. By Lemma $2.3 \mathrm{iv} \tilde{M}_{\sigma}$ is a quasi-Hamiltonian $\tilde{K}_{\sigma}$-manifold with a moment map $\tilde{\Phi}_{\sigma}: \tilde{M}_{\sigma} \rightarrow \tilde{\mathrm{K}}_{\sigma}$ obtained by lifting $\Phi_{\sigma}^{\prime}$. By Lemma 2.3 iii) the map $\pi_{1} \circ \tilde{\Phi}_{\sigma}$ is a moment map for the $\mathrm{K}_{1}$-action on $\tilde{M}_{\sigma}$, where $\pi_{1}: \tilde{K}_{\sigma} \rightarrow K_{1}$ is the 
projection. Consider the quasi-symplectic quotient

$$
\tilde{X}_{\sigma}=\tilde{M}_{\sigma} / / K_{1}=\left(\pi_{1} \circ \tilde{\Phi}_{\sigma}\right)^{-1}(1) / K_{1}=\tilde{\Phi}_{\sigma}^{-1}\left(K_{2}\right) / K_{1},
$$

a space which, by Theorem 2.9 stratifies naturally into quasi-Hamiltonian $K_{2}$ manifolds. The moment map is the continuous map

$$
\Psi_{2}: \tilde{X}_{\sigma} \rightarrow K_{2}
$$

induced by $\pi_{2} \circ \tilde{\Phi}_{\sigma}: \tilde{M}_{\sigma} \rightarrow K_{2}$. Recall that $X_{\sigma}$ denotes the piece $\Phi^{-1}(\exp \sigma) / K_{2}$ in the decomposition (3.3.

\subsection{Lemma. $\quad$ (i) $\tilde{\Phi}_{\sigma}^{-1}\left(K_{2}\right)=\pi_{\sigma}^{-1} \Phi^{-1}(\exp \sigma)$.}

(ii) The $\Gamma_{\sigma}$-action on $\tilde{M}_{\sigma}$ descends to a free action on $\tilde{X}_{\sigma}$.

(iii) $\pi_{\sigma}$ induces a normal covering map $\pi_{\sigma}: \tilde{X}_{\sigma} \rightarrow X_{\sigma}$ with covering group $\Gamma_{\sigma}$. The $\mathrm{K}_{2}$-action on $\tilde{X}_{\sigma}$ commutes with the $\Gamma_{\sigma}$-action. The $\mathrm{K}_{2}$-moment map (3.9) and the reduced symplectic forms on the strata of $\tilde{X}_{\sigma}$ are $\Gamma_{\sigma}$-invariant.

Proof. (1) follows from Lemma 3.7 iil and the fact that $\tilde{\Phi}_{\sigma}$ is the lift of $\Phi_{\sigma}^{\prime}$.

By definition $\tilde{M}_{\sigma}$ is the fibred product

$$
\tilde{M}_{\sigma}=\left\{\left(x, k_{1}, k_{2}\right) \mid x \in M_{\sigma}, k_{1} \in K_{1}, k_{2} \in K_{2}, \Phi_{\sigma}^{\prime}(x)=k_{1} k_{2}\right\} .
$$

The action of $g \in \Gamma_{\sigma}$ is given by $g \cdot\left(x, k_{1}, k_{2}\right)=\left(x, g k_{1}, g^{-1} k_{2}\right)$, whereas the action of $k \in K_{1}$ is given by $k \cdot\left(x, k_{1}, k_{2}\right)=\left(k x, k k_{1} k^{-1}, k_{2}\right)$. Therefore $g \cdot\left(x, k_{1}, k_{2}\right)=$ $k \cdot\left(x, k_{1}, k_{2}\right)$ implies $g=1$, so $\Gamma_{\sigma}$ acts freely on the orbit space $\tilde{X}_{\sigma}=\tilde{M}_{\sigma} / K_{1}$.

The first statement in (iii) follows immediately from (ii) and (iii). Since $\Gamma_{\sigma}$ is central in $\tilde{\mathrm{K}}_{\sigma}$, its action on $\tilde{X}_{\sigma}$ commutes with that of $K_{2}$ and $\Psi_{2}$ is $\Gamma_{\sigma}$-invariant. The lift $\tilde{\omega}_{\sigma}$ of the two-form $\omega_{\sigma}$ on $M_{\sigma}$ is $\tilde{K}_{\sigma}$-invariant, so the induced forms on the strata of $\tilde{X}_{\sigma}$ are $\Gamma_{\sigma}$-invariant.

Thus we have shown that each of the subspaces $X_{\sigma} \subseteq M_{\text {impl }}$ can be written, up to a finite covering map, as a quasi-symplectic quotient. Recall from Theorem 2.9 that the covering space $\tilde{X}_{\sigma}$ stratifies according to $K_{1}$-orbit type into symplectic manifolds. Because $\Gamma_{\sigma}$ is central in $K_{1}$, this stratification is $\Gamma_{\sigma}$-invariant. From Lemma 3.10 iii we now obtain the following.

3.12. Corollary. $X_{\sigma}$ stratifies into quasi-Hamiltonian $\mathrm{K}_{2}$-manifolds. The strata are of the form $\mathrm{S} / \Gamma_{\sigma}$, where $\mathrm{S}$ runs over the strata of $\tilde{\mathrm{X}}_{\sigma}$.

The $K_{2}$-moment map sends $X_{\sigma}$ into $g_{\sigma}^{-1} \exp \sigma \subseteq K_{2}$. Moreover, the $K_{2}$-action on $X_{\sigma}$ extends naturally to the maximal torus $T$ in the following way. First extend the $\mathrm{K}_{2}$-action on $\tilde{X}_{\sigma}$ to a quasi-Hamiltonian action of the maximal torus $\tilde{T}_{\sigma}=\left(K_{1} \cap\right.$ $\mathrm{T}) \times \mathrm{K}_{2}$ of $\tilde{\mathrm{K}}_{\sigma}$ by letting the factor $\mathrm{K}_{1} \cap \mathrm{T}$ act trivially. As moment map $\tilde{\Psi}: \tilde{X}_{\sigma} \rightarrow \tilde{\mathrm{T}}_{\sigma}$ we take $\tilde{\Psi}=\left(\Psi_{1}, \Psi_{2}\right)$, where $\Psi_{1}: \tilde{X}_{\sigma} \rightarrow K_{1} \cap T$ is the constant map sending $\tilde{X}_{\sigma}$ to $g_{\sigma} \in K_{1} \cap Z\left(K_{\sigma}\right) \subseteq K_{1} \cap T$ and $\Psi_{2}$ is as in (3.9). Then $\tilde{\Psi}$ is $\Gamma_{\sigma}$-equivariant and so induces a moment map $\Psi$ for the action of $T=\tilde{T}_{\sigma} / \Gamma_{\sigma}$ on $X_{\sigma}$, as in the diagram

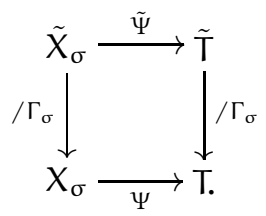


3.13. Lemma. $\Psi$ is equal to the restriction to $X_{\sigma}$ of the imploded moment map $\Phi_{\text {impl }}$.

Proof. We need to show that the following diagram commutes:

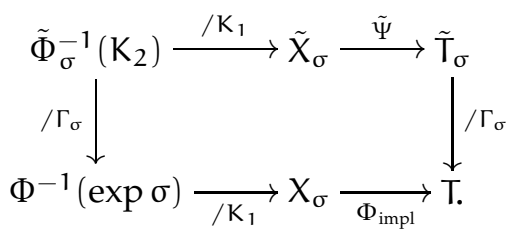

The moment map on the fibred product 3.11 is the projection $\tilde{\Phi}_{\sigma}\left(x, k_{1}, k_{2}\right)=$ $\left(k_{1}, k_{2}\right)$. Therefore

$$
\tilde{\Phi}_{\sigma}^{-1}\left(K_{2}\right)=\left\{\left(x, 1, k_{2}\right) \mid x \in M_{\sigma}, k_{2} \in K_{2}, \Phi_{\sigma}^{\prime}(x)=k_{2}\right\},
$$

and the composition of the two horizontal arrows at the top of (3.14) is the map $\left(x, 1, k_{2}\right) \mapsto\left(g_{\sigma}, \Phi_{\sigma}^{\prime}(x)\right)$. Composing with the quotient map $\tilde{T}_{\sigma} \rightarrow T$, which is given by multiplication, we get the map

$$
\left(x, 1, k_{2}\right) \mapsto g_{\sigma} \Phi_{\sigma}^{\prime}(x)=\Phi_{\sigma}(x)=\Phi(x) .
$$

By definition of the imploded moment map, the composition of the bottom two arrows in (3.14) is the map sending $x \in \Phi^{-1}(\exp \sigma)$ to $\Phi(x)$. Precomposing with the covering map $\tilde{\Phi}_{\sigma}^{-1}\left(K_{2}\right) \rightarrow \Phi^{-1}(\exp \sigma)$, which sends $\left(x, k_{1}, k_{2}\right)$ to $x$, we get the map $\left(x, 1, k_{2}\right) \mapsto \Phi(x)$, which is the same as (3.15).

Let $\left\{X_{i} \mid i \in I\right\}$ be the collection of all strata of all the pieces $X_{\sigma}$, where $\sigma$ ranges over all faces of the alcove. The imploded cross-section is their disjoint union,

$$
M_{\text {impl }}=\coprod_{i \in I} X_{i}
$$

and we shall call the $X_{i}$ the strata of $M_{\text {impl }}$ (although we shall not prove here that they form a stratification of $M_{\text {impl }}$ in the technical sense). The preceding results can be summarized as follows.

3.17. Theorem. The decomposition (3.16) of the imploded cross-section is a locally finite partition into locally closed subspaces, each of which is a symplectic manifold. There is a unique open stratum, which is dense in $\mathrm{M}_{\mathrm{impl}}$ and symplectomorphic to the principal cross-section of $\mathrm{M}$. The action of the maximal torus $\mathrm{T}$ on $\mathrm{M}_{\mathrm{impl}}$ preserves the decomposition and the imploded moment map $\Phi_{\text {impl }}: M_{\text {impl }} \rightarrow$ T restricts to a moment map for the T-action on each stratum.

For this reason we call $M_{\text {impl }}$ a stratified quasi-Hamiltonian T-space. Quotients of $M_{\text {impl }}$ by the T-action can be defined just as if $M_{\text {impl }}$ was a manifold: for $g \in$ $\Phi(M) \cap \exp \overline{\mathcal{A}}=\Phi_{\text {impl }}\left(M_{\text {impl }}\right)$ one puts $M_{\text {impl }} / / g^{T}=\Phi_{\text {impl }}^{-1}(g) / T$. Again these quotients decompose into symplectic manifolds according to orbit type. This is seen by applying Theorem 2.9 to all of the T-manifolds $X_{i}$ that intersect $\Phi_{\text {impl }}^{-1}(\mathrm{~g})$. Moreover, $\Phi_{\text {impl }}^{-1}(\mathrm{~g})$ is the quotient of $\Phi^{-1}(\mathrm{~g})$ by $\left[\mathrm{K}_{\mathrm{g}}, \mathrm{K}_{\mathrm{g}}\right]$ and the quotient map $\Phi^{-1}(\mathrm{~g}) \rightarrow \Phi_{\text {impl }}^{-1}(\mathrm{~g})$ descends to a homeomorphism $M / / \mathrm{g} \mathrm{K} \rightarrow \mathrm{M}_{\mathrm{impl}} / / \mathrm{g} \mathrm{T}$. It is now straightforward to check the following assertion. 
3.18. Addendum. For all $\mathrm{g} \in \Phi(\mathrm{M}) \cap \exp \overline{\mathcal{A}}$, the homeomorphism $\mathrm{M} / / \mathrm{g} \mathrm{K} \rightarrow \mathrm{M}_{\mathrm{impl}} / \mathrm{g}_{\mathrm{g}} \mathrm{T}$ induced by the $\left[\mathrm{K}_{\mathrm{g}}, \mathrm{K}_{\mathrm{g}}\right]$-orbit map $\Phi^{-1}(\mathrm{~g}) \rightarrow \Phi_{\mathrm{impl}}^{-1}(\mathrm{~g})$ sends strata to strata and restricts to a symplectomorphism on each stratum.

Thus the imploded cross-section $M_{\text {impl }}$ is the "abelianization" of $M$ in the sense that every quasi-symplectic quotient of $M$ by the K-action can be written as a quasi-symplectic quotient of $\mathrm{M}_{\mathrm{impl}}$ by the T-action.

Just like quasi-Hamiltonian reduction, quasi-Hamiltonian implosion for the action of a product $\mathrm{K}_{1} \times \mathrm{K}_{2}$ can be performed with respect to one of the factors, say $\mathrm{K}_{2}$. Using Remark 3.5 one can show the resulting space is quasi-Hamiltonian for the product $K_{1} \times T_{2}$, where $T_{2}$ is a maximal torus of $K_{2}$. We shall not develop this variant of the theory save in the important special case of the double DK, which is investigated in the next section.

\section{THE UNIVERSAL IMPLODED CROSS-SECTION}

Implosion of the double. The cotangent bundle $\mathrm{T}^{*} \mathrm{~K}$ is a Hamiltonian $\mathrm{K} \times \mathrm{K}$ manifold. Implosion with respect to, say, the right $\mathrm{K}$-action yields a $\mathrm{K} \times \mathrm{T}$-space $\mathrm{T}^{*} \mathrm{~K}_{\mathrm{impl}}$, which stratifies into symplectic manifolds. This space is the universal imploded cross-section in the sense that the imploded cross-section of every Hamiltonian $\mathrm{K}$-manifold $\mathrm{M}$ is a quotient,

$$
\mathrm{M}_{\mathrm{impl}} \cong\left(\mathrm{M} \times \mathrm{T}^{*} \mathrm{~K}_{\mathrm{impl}}\right) / / \mathrm{K} \text {. }
$$

Here the quotient is taken with respect to the given $\mathrm{K}$-action on $\mathrm{M}$ and the residual (left) K-action on $\mathrm{T}^{*} \mathrm{~K}_{\text {impl }}$. (See [GJS02, Theorem 4.9].) A similar phenomenon occurs in the quasi-Hamiltonian category. According to AMM98, Section 3.2] the quasi-Hamiltonian analogue of the cotangent bundle $\mathrm{T}^{*} \mathrm{~K}$ is the double $\mathrm{DK}=\mathrm{K} \times$ $K$. The $K \times K$-action on DK is defined by $\left(g_{1}, g_{2}\right)(u, v)=\left(g_{1} u g_{2}^{-1}, \operatorname{Ad}\left(g_{2}\right) v\right)$, the two-form by

$$
\omega=-\frac{1}{2}\left(\operatorname{Ad}(v) u^{*} \theta_{L}, u^{*} \theta_{L}\right)-\frac{1}{2}\left(u^{*} \theta_{L}, v^{*}\left(\theta_{L}+\theta_{R}\right)\right),
$$

and the moment map $\Psi: \mathrm{DK} \rightarrow \mathrm{K} \times \mathrm{K}$ by $\Psi=\Psi_{1} \times \Psi_{2}$ with

$$
\Psi_{1}(u, v)=\operatorname{Ad}(u) v^{-1} \quad \text { and } \quad \Psi_{2}(u, v)=v .
$$

(Here we work in the coordinate system of AMM98 Remark 3.2]. Actually [loc. cit.] uses the opposite space $\mathrm{DK}^{-}=(\mathrm{K} \times \mathrm{K},-\omega, i \circ \Psi)$, but $\mathrm{DK}^{-}$is isomorphic to DK via the diffeomorphism $(u, v) \mapsto\left(u, v^{-1}\right)$.)

The fusion product $M \circledast D K$ (see Section 2 ) of an arbitrary quasi-Hamiltonian Kmanifold $\mathrm{M}$ and the quasi-Hamiltonian $\mathrm{K} \times \mathrm{K}$-manifold $\mathrm{DK}$ is a quasi-Hamiltonian $\mathrm{K} \times \mathrm{K}$-manifold. Likewise, for each face $\sigma \leq \mathcal{A}$ the cross-section $\Psi_{2}^{-1}\left(\mathfrak{S}_{\sigma}\right)=\mathrm{K} \times \mathfrak{S}_{\sigma}$ of DK is a quasi-Hamiltonian $\mathrm{K} \times \mathrm{K}_{\sigma}$-manifold (see Remark 3.5) and so $\mathrm{M} \circledast\left(\mathrm{K} \times \mathfrak{S}_{\sigma}\right)$ is a quasi-Hamiltonian $\mathrm{K} \times \mathrm{K}_{\sigma}$-manifold.

Now define $j: M \rightarrow M \circledast D K$ by $j(m)=(m, 1, \Phi(m))$. The following result is analogous to [GJS02. Lemma 4.8] and is proved in the same way. (An embedding is quasi-symplectic if it preserves the two-forms.)

4.3. Lemma. (i) The map $\mathrm{j}$ is a quasi-symplectic embedding and induces an isomorphism of quasi-Hamiltonian K-manifolds

$$
\bar{\jmath}: M \rightarrow(M \circledast D K) / / K .
$$


Here the right-hand side is the quotient with respect to the diagonal $\mathrm{K}$-action, with $\mathrm{K}$ acting on the left on DK. The K-action on $(\mathrm{M} \circledast \mathrm{DK}) / / \mathrm{K}$ is the one induced by the right action on DK.

(ii) For every $\sigma \leq \mathcal{A}, j$ maps $M_{\sigma}$ into $M \circledast\left(K \times \mathfrak{S}_{\sigma}\right)$ and induces an isomorphism of quasi-Hamiltonian $\mathrm{K}_{\sigma}$-manifolds

$$
\bar{\jmath}_{\sigma}: M_{\sigma} \rightarrow\left(M \circledast\left(K \times \mathfrak{S}_{\sigma}\right)\right) / / \mathrm{K},
$$

where the quotient is taken as in (i).

We now implode DK, but using only the right action of $\mathrm{K}$ and the second component $\Psi_{2}$ of the moment map. In other words we form the quotient topological space of $\Psi_{2}^{-1}(\exp \overline{\mathcal{A}})=\mathrm{K} \times \exp \overline{\mathcal{A}}$ by the equivalence relation $(u, v) \sim\left(\mathrm{ug}^{-1}, v\right)$ for $g \in\left[K_{v}, K_{v}\right]$. As in (3.3) the resulting space is a union of subspaces,

$$
D K_{\text {impl }}=\coprod_{\sigma \leq \mathcal{A}} \mathrm{K} /\left[\mathrm{K}_{\sigma}, \mathrm{K}_{\sigma}\right] \times \exp \sigma .
$$

The moment map $\Psi_{2}$ is transverse to all faces of the alcove, so it happens that each piece $X_{\sigma}=K /\left[K_{\sigma}, K_{\sigma}\right] \times \exp \sigma$ in this partition is a smooth manifold. We shall show that $X_{\sigma}$ is a quasi-Hamiltonian $\mathrm{K} \times \mathrm{T}$-manifold by writing it as a quasisymplectic quotient up to a covering. Namely let $D K_{\sigma}=\Psi_{2}^{-1}\left(\mathfrak{S}_{\sigma}\right)=\mathrm{K} \times \mathfrak{S}_{\sigma}$. By Remark 3.5 $\mathrm{DK}_{\sigma}$ is a Hamiltonian $\mathrm{K} \times \mathrm{K}_{\sigma}$-manifold with moment map $\Psi_{\sigma}$ being the restriction of $\Psi$ to $D K_{\sigma}$. Let $p: \tilde{K}_{\sigma} \rightarrow K_{\sigma}$ be the covering (3.8) of the centralizer $\mathrm{K}_{\sigma}$. The pullback of this covering via $\Psi_{\sigma}$ is $\mathrm{DK}_{\sigma}^{\sim}=\mathrm{K} \times \tilde{\mathfrak{S}}_{\sigma}$, where $\tilde{\mathfrak{S}}_{\sigma}=\mathrm{p}^{-1}\left(\mathfrak{S}_{\sigma}\right)$. Thus $\mathrm{K} \times \tilde{\mathfrak{S}}_{\sigma}$ is a quasi-Hamiltonian $\mathrm{K} \times \tilde{\mathrm{K}}_{\sigma}$-manifold. Its quotient with respect to the $\left[\mathrm{K}_{\sigma}, \mathrm{K}_{\sigma}\right]$-action is the quasi-Hamiltonian $\mathrm{K} \times \mathrm{Z}\left(\mathrm{K}_{\sigma}\right)^{0}$-manifold

$$
\tilde{X}_{\sigma}=\left(\mathrm{K} \times \tilde{\mathfrak{S}}_{\sigma}\right) / /\left[\mathrm{K}_{\sigma}, \mathrm{K}_{\sigma}\right]=\mathrm{K} /\left[\mathrm{K}_{\sigma}, \mathrm{K}_{\sigma}\right] \times \mathrm{p}^{-1}(\exp \sigma)
$$

which is a covering space of $X_{\sigma}$ with covering group $\Gamma_{\sigma}$. The following is an analogue of Corollary 3.12 and Lemma 3.13 and is proved in the same way.

4.5. Lemma. For every $\sigma \leq \mathcal{A}$ the subspace $\mathrm{X}_{\sigma}=\mathrm{K} /\left[\mathrm{K}_{\sigma}, \mathrm{K}_{\sigma}\right] \times \exp \sigma$ of $\mathrm{DK}_{\mathrm{impl}}$ is a quasi-Hamiltonian $\mathrm{K} \times \mathrm{T}$-manifold. The moment map $\mathrm{X}_{\sigma} \rightarrow \mathrm{K} \times \mathrm{T}$ is the restriction to $\mathrm{X}_{\sigma}$ of the continuous map $\Psi_{\mathrm{impl}}: \mathrm{DK}_{\mathrm{impl}} \rightarrow \mathrm{K} \times \mathrm{T}$ induced by $\Psi: \mathrm{DK} \rightarrow \mathrm{K} \times \mathrm{K}$.

We can now establish the universal property of $\mathrm{DK}_{\mathrm{impl}}$. Consider an arbitrary quasi-Hamiltonian $\mathrm{K}$-manifold $(M, \omega, \Phi)$. We form the fusion product $M \circledast D K_{\text {impl }}$

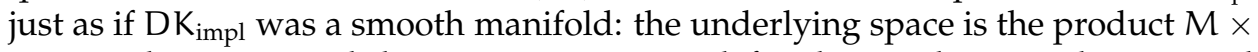
$\mathrm{DK}_{\mathrm{impl}}$, the action and the moment map are defined as in the smooth case, and the two-form is defined stratum by stratum. The resulting space is a stratified space whose strata are quasi-Hamiltonian $\mathrm{K} \times \mathrm{T}$-manifolds. Stratum by stratum we can reduce $M \circledast D K_{\text {impl }}$ with respect to the $\mathrm{K}$-action to obtain a stratified quasiHamiltonian T-space $\left(M \circledast D K_{\text {impl }}\right) / / K$. This quotient turns out to be the same as $\mathrm{M}_{\text {impl }}$.

4.6. Theorem (universality of the imploded double). Let $M$ be a quasi-Hamiltonian $\mathrm{K}$-manifold. The map $\mathrm{j}$ induces a homeomorphism

$$
\mathrm{j}_{\mathrm{impl}}: \mathrm{M}_{\mathrm{impl}} \cong\left(\mathrm{M} \circledast \mathrm{DK} \mathrm{K}_{\mathrm{impl}}\right) / / \mathrm{K},
$$

which maps strata to strata and whose restriction to each stratum is an isomorphism of quasi-Hamiltonian T-manifolds. 
Proof. Observe that $j$ maps $\Phi^{-1}(\exp \mathcal{A})$ into $M \times \Phi_{2}^{-1}(\exp \mathcal{A})$ and that $\mathrm{m}_{1} \sim \mathrm{m}_{2}$ implies $j\left(m_{1}\right)=j\left(m_{2}\right)$ for all $m_{1}, m_{2} \in \Phi^{-1}(\exp \mathcal{A})$. Hence $j$ descends to a continuous map j': $\mathrm{M}_{\mathrm{impl}} \rightarrow \mathrm{M} \circledast \mathrm{DK}_{\mathrm{impl}}$. The $\mathrm{K} \times \mathrm{T}$-moment map on $\mathrm{M} \circledast \mathrm{DK}_{\mathrm{impl}}$ is $\phi=$ $\phi_{1} \times \phi_{2}$, where $\phi_{1}(\mathrm{~m},(\mathfrak{u}, v) \bmod \sim)=\Phi(\mathrm{m}) \mathfrak{u} v^{-1} u^{-1}$ and $\phi_{2}(\mathrm{~m},(\mathfrak{u}, v) \bmod \sim)=$ $v$. Therefore $j^{\prime}$ maps $M_{\text {impl }}$ into $\phi_{1}^{-1}(1)$. Dividing by the action of $K$ we obtain the map $j_{\text {impl }}$. It is straightforward to check that $j_{\text {impl }}$ is bijective and therefore

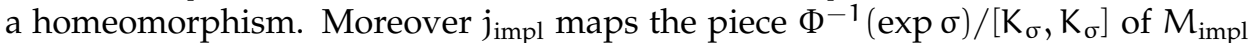
onto $\left(M \circledast X_{\sigma}\right) / / K$ (where $X_{\sigma}$ is as in Lemma 4.5). It remains to show that the restriction of $j_{\text {impl }}$,

$$
\Phi^{-1}(\exp \sigma) /\left[\mathrm{K}_{\sigma}, \mathrm{K}_{\sigma}\right] \rightarrow\left(\mathrm{M} \circledast \mathrm{X}_{\sigma}\right) / / \mathrm{K},
$$

maps strata to strata and is an isomorphism of quasi-Hamiltonian T-manifolds on each stratum. To see this, recall the isomorphism of Lemma 4.3 iii, which lifts to an isomorphism of quasi-Hamiltonian $\tilde{\mathrm{K}}_{\sigma}$-manifolds

$$
\tilde{\mathrm{M}}_{\sigma} \rightarrow\left(\mathrm{M} \circledast\left(\mathrm{K} \times \tilde{\mathfrak{S}}_{\sigma}\right)\right) / / \mathrm{K} .
$$

Upon reduction by $\left[\mathrm{K}_{\sigma}, \mathrm{K}_{\sigma}\right]$ this yields a stratification-preserving homeomorphism

$$
\tilde{\mathrm{M}}_{\sigma} / /\left[\mathrm{K}_{\sigma}, \mathrm{K}_{\sigma}\right] \rightarrow\left(\mathrm{M} \circledast \tilde{\mathrm{X}}_{\sigma}\right) / / \mathrm{K},
$$

whose restriction to each stratum is an isomorphism of $\mathrm{K} \times \mathrm{Z}\left(\mathrm{K}_{\sigma}\right)^{0}$-spaces. Taking the quotient by $\Gamma_{\sigma}$ on both sides we obtain the map 4.7. It now follows from Corollary 3.12 that this map sends strata isomorphically onto strata.

Discrete symmetries. The centre of $K$ gives rise to a set of discrete symmetries of the universal imploded cross-section $D K_{\text {impl }}$. A stratum $K /\left[K_{\sigma}, K_{\sigma}\right] \times \exp \sigma$ of $D K_{\text {impl }}$ consists of a single point if and only if $\sigma$ is a vertex of the alcove and exp $\sigma$ is an element of the centre $Z(K)$. Thus one-point strata are in one-to-one correspondence with the centre. These strata are exactly the fixed points under the $\mathrm{K} \times \mathrm{T}$ action. It turns out there exists an extension $\uparrow$ of the centre by the maximal torus that acts on $\mathrm{DK}_{\mathrm{impl}}$, permutes the one-point strata, and in addition preserves the quasi-Hamiltonian structure. This action has no Hamiltonian analogue, because $\mathrm{T}^{*} \mathrm{~K}_{\text {impl }}$ has just a single one-point stratum (the "vertex"). The material below relies on facts concerning the action of the centre on the alcove, which are reviewed at the end of Appendix

Let $\zeta: Z(K) \rightarrow W$ be the injective homomorphism of Lemma $A .16$ and let $\uparrow$ be the preimage of $\zeta(Z(K))$ under the canonical projection $N(T) \rightarrow W$, where $N(T)$ is the normalizer of the maximal torus. Thus $\uparrow$ is a subgroup of $N(T)$ and we have short exact sequence

$$
\mathrm{T} \longleftrightarrow \text { 个 } \stackrel{\pi}{\longrightarrow} \mathrm{Z}(\mathrm{K}) .
$$

4.9. Example. The centre of $K=\mathbf{S U}(l+1)$ is generated by $c=e^{-2 \pi i /(l+1)} \mathrm{I}_{l+1}$, where $I_{l+1}$ is the identity matrix, and by Example A.18 $\zeta$ (c) is the cyclic permutation $x \mapsto\left(x_{l+1}, x_{1}, x_{2} \ldots, x_{l}\right)$. A representative of $c$ in $\uparrow$ is the matrix

$$
\widehat{c}=-\left(\begin{array}{cc}
0 & (-1)^{l} \\
I_{l} & 0
\end{array}\right),
$$


and therefore $\hat{\top}=\bigcup_{j=0}^{l} \hat{c}^{j} T$. Note that $\hat{T}$ is not abelian. For $t \in T$ the order of $\hat{c} t$ is $l+1$ if $l$ is even and $2 l+2$ if $l$ is odd. Hence the sequence 4.8 splits if and only if $l$ is even.

Define a smooth $\widehat{\top}$-action $\mathfrak{A}$ on DK by

$$
\mathfrak{A}_{\mathrm{g}}(\mathrm{u}, v)=\left(\mathrm{ug}^{-1}, \pi(\mathrm{g}) \operatorname{Ad}(\mathrm{g}) v\right)=\left(\mathrm{ug}^{-1}, \mathrm{~g} \pi(\mathrm{g}) v \mathrm{~g}^{-1}\right) .
$$

Since $\mathrm{T}=\operatorname{ker} \pi$ this action is an extension of the right T-action on DK.

4.10. Lemma. The 个-action preserves $\Psi_{2}^{-1}(\exp \overline{\mathcal{A}})=\mathrm{K} \times \exp \overline{\mathcal{A}}$ and induces a continuous effective action on $\mathrm{DK}_{\text {impl }}$. This induced action commutes with the $\mathrm{K}$-action, maps strata to strata, and is transitive on the collection of one-point strata.

Proof. If $v \in \exp \overline{\mathcal{A}}$ then $\pi(\mathrm{g}) \mathrm{gvg}^{-1} \in \exp \overline{\mathcal{A}}$ by Lemma A.16 (applied to $w=g \mathrm{~T} \in$ $W$ and $c=\pi(g))$, so $\mathfrak{A}_{\mathrm{g}}$ maps $\Psi_{2}^{-1}(\exp \overline{\mathcal{A}})$ to itself. Let $(u, v) \in \mathrm{K} \times \exp \overline{\mathcal{A}}$ and $k \in\left[K_{v}, K_{v}\right]$. Then $(u, v)$ is equivalent to $\left(u k^{-1}, v\right)$ and

$$
\mathfrak{A}_{\mathrm{g}}\left(u k^{-1}, v\right)=\left(u k^{-1} \mathrm{~g}^{-1}, \pi(\mathrm{g}) \operatorname{Ad}(\mathrm{g}) v\right)=\left(\mathrm{ug}^{-1} \operatorname{Ad}(\mathrm{g}) k^{-1}, \pi(\mathrm{g}) \operatorname{Ad}(\mathrm{g}) v\right),
$$

where

$$
\operatorname{Ad}(g) k \in \operatorname{Ad}(g)\left[K_{v}, K_{v}\right]=\left[K_{\pi(g)} \operatorname{Ad}(g) v, K_{\pi(g) \operatorname{Ad}(g) v}\right],
$$

so $\mathfrak{A}_{g}(u, v) \sim f_{g}\left(u k^{-1}, v\right)$. Hence $\mathfrak{A}_{g}$ descends to a homeomorphism $\overline{\mathfrak{A}}_{g}$ from $\mathrm{DK}_{\text {impl }}$ to itself. On the open stratum $\mathrm{K} \times \exp \overline{\mathcal{A}} \mathrm{g}$ acts by right translation on $\mathrm{K}$ and via the central element $\pi(g)$ on $\mathcal{A}$. This shows that the induced action is effective. Since the action $\mathfrak{A}$ commutes with the left K-action on DK, $\overline{\mathfrak{A}}$ commutes with the induced $\mathrm{K}$-action on $\mathrm{DK}_{\text {impl }}$. The action of the coweight lattice $P\left(R^{\vee}\right)=\exp _{T}^{-1}(Z(K))$ on $t$ is affine and maps alcoves to alcoves. (See the proof of Lemma A.16) Therefore it maps faces of alcoves to faces of alcoves. This implies that $\overline{\mathfrak{A}}_{\mathrm{g}}$ maps strata to strata. A one-point stratum is an equivalence class $(1, c) \bmod \sim$ for some $c \in Z(K)$. Since $\mathfrak{A}_{\mathrm{g}}(1, \mathrm{c})=\left(\mathrm{g}^{-1}, \pi(\mathrm{g}) \mathrm{c}\right) \sim(1, \pi(\mathrm{g}) \mathrm{c})$, we have $\overline{\mathfrak{A}}_{\mathrm{g}}((1, \mathrm{c}) \bmod \sim)=((1, \pi(\mathrm{g}) \mathrm{c}) \bmod \sim)$, so 个 acts transitively on the set of one-point strata.

Observe however that the centre $Z(K)$ itself does not act on $D K_{\text {impl }}$ (unless the sequence (4.8) splits). Also, $\mathrm{DK}_{\text {impl }}$ is not quite a quasi-Hamiltonian $\mathrm{K} \times \mathrm{T}$ manifold. The conjugation action of $\mathrm{K} \times \mathrm{T}$ on $\mathrm{K} \times \mathrm{T}$ is given by

$$
\operatorname{Ad}(k, g) \cdot\left(g_{1}, g_{2}\right)=\left(\operatorname{Ad}(k) g_{1}, \operatorname{Ad}(g) g_{2}\right) \text {, }
$$

whereas the imploded moment map $\Psi_{\text {impl }}$ is equivariant only under the "affine" conjugation action $\operatorname{Ad}^{\wedge}(k, g)\left(g_{1}, g_{2}\right)=\left(\pi(g)^{-1} \operatorname{Ad}(k) g_{1}, \pi(g) \operatorname{Ad}(g) v\right)$.

4.11. Proposition. The $\mathrm{K} \times \mathrm{T}$-action on $\mathrm{DK}_{\text {impl }}$ preserves the two-forms on the strata. The $\mathrm{K} \times \mathrm{T}$-moment map $\Psi_{\text {impl }}: \mathrm{DK}_{\mathrm{impl}} \rightarrow \mathrm{K} \times \mathrm{T}$ is $\mathrm{Ad}^{\wedge}$-equivariant for the $\mathrm{K} \times \mathrm{T}$-action.

Proof. The map $\mathfrak{A}_{\mathrm{g}}$ is the composition of $(u, v) \mapsto\left(\mathrm{ug}^{-1}, \operatorname{Ad}(\mathrm{g}) v\right)$ and $(u, v) \mapsto$ $(u, \pi(g) v)$. The first map is the right action of the element $g$ and therefore preserves $\omega$; the second map preserves $\omega$ because both $\theta_{L}$ and $\theta_{R}$ are invariant under translation by the central element $\pi(\mathrm{g})$. It follows that $\overline{\mathfrak{A}}_{\mathrm{g}}$ preserves the forms on the strata. For $(k, g) \in \mathrm{K} \times \widehat{T}$ and $(u, v) \in \mathrm{K} \times \exp \overline{\mathcal{A}}$ we have

$$
\begin{aligned}
\Psi((k, g) \cdot(u, v))=\Psi & \left(k u g^{-1}, \pi(g) \operatorname{Ad}(g) v\right) \\
& =\left(\pi(g)^{-1} \operatorname{Ad}(k u) v^{-1}, \pi(g) \operatorname{Ad}(g) v\right)=\operatorname{Ad}^{`}(k, g) \Psi(u, v),
\end{aligned}
$$


which establishes the equivariance of $\Psi$.

Further discrete symmetries of the universal imploded cross-section come from the duality automorphism of the alcove, which is defined by $\xi \mapsto-w_{0} \xi$, where $w_{0}$ is the longest Weyl group element. Let $T_{0}$ be the inverse image of the subgroup $\left\langle w_{0}\right\rangle=\left\{1, w_{0}\right\}$ of $W$ under the projection $\mathrm{N}(\mathrm{T}) \rightarrow \mathrm{T}$. It fits into an exact sequence

$$
\mathrm{T} \longrightarrow \mathrm{T}_{0} \longrightarrow\left\langle w_{0}\right\rangle \cong \mathbf{Z} / 2 \mathrm{Z} \text {. }
$$

Define a smooth $T_{0}$-action on DK by $\mathfrak{B}_{g}(u, v)=\left(u g^{-1}, \operatorname{Ad}(g) v^{\rho(g)}\right)$, where $\rho(g)=$ 1 if $\mathrm{g}$ projects to $1 \in W$ and $\rho(\mathrm{g})=-1$ if g projects to $w_{0}$. The following result is analogous to Lemma 4.10 and Proposition 4.11.

4.13. Proposition. The $T_{0}$-action preserves $\Psi_{2}^{-1}(\exp \overline{\mathcal{A}})=\mathrm{K} \times \exp \overline{\mathcal{A}}$ and descends to a continuous effective action on $\mathrm{DK}_{\mathrm{impl}}$. This action commutes with the $\mathrm{K}$-action and maps strata to strata. An element $\mathrm{g} \in \mathrm{T}_{0}$ preserves or reverses the two-forms on the strata according to whether $\rho(\mathrm{g})=1$ or $\rho(\mathrm{g})=-1$. The $\mathrm{K} \times \mathrm{T}$-moment map $\Psi_{\mathrm{impl}}: \mathrm{DK}$ impl $\rightarrow$ $\mathrm{K} \times \mathrm{T}$ is $\mathrm{T}_{0}$-equivariant for the $\mathrm{T}_{0}$-action $\mathrm{g} \cdot\left(\mathrm{g}_{1}, \mathrm{~g}_{2}\right)=\left(\mathrm{g}_{1}^{\rho(\mathrm{g})}, \mathrm{Ad}(\mathrm{g}) \mathrm{g}_{2}^{\rho(\mathrm{g})}\right)$ on $\mathrm{K} \times \mathrm{T}$.

4.14. Example. The longest Weyl group element of $K=\mathbf{S U}(l+1)$ is the permutation $w_{0}(x)=\left(x_{l+1}, x_{l}, \ldots, x_{1}\right)$. One checks readily that $w_{0}$ has a representative of order two in $N(T)$ if and only if $l \neq \equiv 1 \bmod 4$; if $l \equiv 1 \bmod 4$ it has a representative of order four. Therefore the sequence (4.12) splits if and only if $l \not \equiv 1 \bmod 4$. A convenient choice for such a representative is

$$
\mathrm{n}_{0}=\left(\begin{array}{cc}
0 & (-1)^{l(l+1) / 2} \\
\mathrm{~J} & 0
\end{array}\right) \quad \text { if } l \equiv 0,1,3 \bmod 4,
$$

where $J_{l}$ is the antidiagonal $l \times l$-matrix with ones up the second diagonal, and

$$
n_{0}=\left(\begin{array}{ccc}
0 & 0 & J_{2 m+1} \\
0 & -1 & 0 \\
J_{2 m+1} & 0 & 0
\end{array}\right) \quad \text { if } l=4 m+2 \equiv 2 \bmod 4 .
$$

For $l=1$ we have $T_{0}=\hat{T}=N(T)$, but the actions of $T_{0}$ and $\hat{T}$ on $D K_{i m p l}$ are not the same.

A smoothness criterion. Certain strata of the universal imploded cross-section are redundant in the sense that $\mathrm{DK}_{\mathrm{impl}}$ is a smooth manifold at these points and the two-form and the moment map extend smoothly. Thus these singularities are removable in the strongest possible sense. For instance if $\mathrm{K}$ is a product of copies of $\mathbf{S U}(2)$ all singularities are removable. To prove this we must be more explicit about the analogy between the double DK and the cotangent bundle $\mathrm{T}^{*} \mathrm{~K}$.

Identify $\mathfrak{k}^{*}$ with $\mathfrak{k}$ by means of the inner product, trivialize $T^{*} K$ by means of left translations, and let $\mathcal{H}$ be the $\mathrm{K} \times \mathrm{K}$-equivariant map id $\times \exp : \mathrm{T}^{*} \mathrm{~K} \cong \mathrm{K} \times \mathfrak{k} \rightarrow \mathrm{DK}$. Let $\omega_{0}$ be the standard symplectic form on $T^{*} K$ and $\Psi_{0}(g, \lambda)=(-\operatorname{Ad}(g) \lambda, \lambda)$ the standard moment map for the $\mathrm{K} \times \mathrm{K}$-action. Let $\mathrm{O}$ be the set of all $\xi \in \mathfrak{t}$ with $(2 \pi i)^{-1} \alpha(\xi)<1$ for all roots $\alpha$ and let $U=\operatorname{Ad}(K) O$.

4.15. Proposition. The triple $\left(T^{*} K, \mathcal{H}^{*} \omega, \mathcal{H}^{*} \Psi\right)$ is the exponentiation (see Section 2] of the Hamiltonian $\mathrm{K} \times \mathrm{K}$-manifold $\left(\mathrm{T}^{*} \mathrm{~K}, \omega_{0}, \Psi_{0}\right)$. Hence $\left(\mathrm{K} \times \mathrm{U}, \mathcal{H}^{*} \omega, \mathcal{H}^{*} \Psi\right)$ is a quasiHamiltonian $\mathrm{K} \times \mathrm{K}$-manifold. 
Proof. A tangent vector to $\mathrm{T}^{*} \mathrm{~K}=\mathrm{K} \times \mathfrak{k}$ at $(\mathrm{g}, \lambda)$ is of the form $\left(\mathrm{L}(\mathrm{g})_{*} \xi, \eta\right)$ with $\xi$, $\eta \in \mathfrak{k}$. The standard symplectic form is

$$
\left(\omega_{0}\right)_{(g, \lambda)}\left(\left(L(g)_{*} \xi_{1}, \eta_{1}\right),\left(L(g)_{*} \xi_{2}, \eta_{2}\right)\right)=\left(\xi_{2}, \eta_{1}\right)-\left(\xi_{1}, \eta_{2}\right) .
$$

A calculation using (2.5) and (4.1) yields

$$
\begin{aligned}
\mathcal{H}^{*} \omega_{(g, \lambda)}\left(\left(\mathrm{L}(\mathrm{g})_{*} \xi_{1}, \eta_{1}\right),\left(\mathrm{L}(\mathrm{g})_{*} \xi_{2}, \eta_{2}\right)\right)=-\left((\sinh \operatorname{ad} \lambda) \xi_{1}, \xi_{2}\right) \\
-\left(\frac{\sinh \operatorname{ad} \lambda}{\operatorname{ad} \lambda} \xi_{1}, \eta_{2}\right)+\left(\frac{\sinh \operatorname{ad} \lambda}{\operatorname{ad} \lambda} \xi_{2}, \eta_{1}\right) .
\end{aligned}
$$

Let $\pi_{1}, \pi_{2}: \mathfrak{k} \oplus \mathfrak{k} \rightarrow \mathfrak{k}$ be the projections onto the respective factors and let $\varpi^{\prime}=$ $\pi_{1}^{*} \varpi+\pi_{2}^{*} \varpi$, where $\varpi \in \Omega^{2}(\mathfrak{k})^{\mathrm{K}}$ is given by $[2.6$. From

$$
\mathrm{T}_{(\mathrm{g}, \lambda)} \Psi_{0}\left(\mathrm{~L}(\mathrm{~g})_{*} \xi, \eta\right)=(-\operatorname{Ad}(\mathrm{g})([\xi, \lambda]+\eta), \eta)
$$

we obtain

$$
\begin{aligned}
\Psi_{0}^{*} \varpi_{(g, \lambda)}^{\prime}( & \left.\left(\mathrm{L}(\mathrm{g})_{*} \xi_{1}, \eta_{1}\right),\left(\mathrm{L}(\mathrm{g})_{*} \xi_{2}, \eta_{2}\right)\right) \\
= & \varpi_{-\lambda}\left(\left[\xi_{1}, \lambda\right]+\eta_{1},\left[\xi_{2}, \lambda\right]+\eta_{2}\right)+\varpi_{\lambda}\left(\eta_{1}, \eta_{2}\right) \\
= & \left((\operatorname{ad} \lambda-\sinh \operatorname{ad} \lambda) \xi_{1}, \xi_{2}\right)+\left(\xi_{1}, \eta_{2}\right)-\left(\xi_{2}, \eta_{1}\right) \\
& \quad-\left(\frac{\sinh \operatorname{ad} \lambda}{\operatorname{ad} \lambda} \xi_{1}, \eta_{2}\right)+\left(\frac{\sinh \operatorname{ad} \lambda}{\operatorname{ad} \lambda} \xi_{2}, \eta_{1}\right) .
\end{aligned}
$$

From (4.16-4.18 we conclude $\mathcal{H}^{*} \omega=\omega_{0}+\Psi_{0}^{*} \varpi^{\prime}$. Let exp $\sim$ denote the exponential map of $K \times K$. Then $\exp ^{\sim}\left(\Psi_{0}(g, \lambda)\right)=(\operatorname{Ad}(g) \exp (-\lambda), \exp \lambda)$ and therefore $\mathcal{H}^{*} \Psi=\exp ^{\sim} \circ \Psi_{0}$. Lastly by Lemma A.8 iii exp: $\mathrm{U} \rightarrow \mathrm{K}$ is a local diffeomorphism onto its image. Therefore $\left(\mathrm{K} \times \mathrm{U}, \mathcal{H}^{*} \omega, \mathcal{H}^{*} \Psi\right)$ is a quasi-Hamiltonian $\mathrm{K} \times \mathrm{K}$-manifold.

The local diffeomorphism $\mathcal{H}=\mathrm{id} \times \exp : \mathrm{K} \times \mathrm{U} \rightarrow \mathrm{K} \times \exp \mathrm{U}$ descends to the imploded cross-sections as follows. Under the isomorphism $\mathfrak{k}^{*} \cong \mathfrak{k}$ the chamber $\mathcal{C}$ is mapped to the dual chamber $\mathcal{C}^{\vee}$. By Lemma A.8 i the intersection $\overline{\mathcal{C}} \cap \mathrm{O}$ is equal to $\overline{\mathcal{A}} \cap \mathrm{O}$ and therefore $\mathcal{H}$ maps $\Psi_{0}^{-1}(\overline{\mathcal{C}}) \cap(\mathrm{K} \times \mathrm{U})$ onto $\Psi^{-1}(\overline{\mathcal{A}}) \cap(\mathrm{K} \times \exp \mathrm{U})$. Moreover $\mathrm{K}_{\xi}=\mathrm{K}_{\exp \xi}$ for $\xi \in \overline{\mathcal{A}} \cap \mathrm{O}$ by Lemma A.3 iii, so $\mathcal{H}$ induces a $\mathrm{K} \times \mathrm{T}$ equivariant surjective local homeomorphism

$$
\mathrm{h}:(\mathrm{K} \times \mathrm{U})_{\mathrm{impl}} \rightarrow(\mathrm{K} \times \exp \mathrm{U})_{\mathrm{impl}} .
$$

The left-hand side is open in the Hamiltonian implosion $T^{*} K_{i m p l}$ and the righthand side is an open and dense subset of the quasi-Hamiltonian implosion $\mathrm{DK}_{\text {impl}}$, namely the union of the strata corresponding to the faces $\sigma \leq \mathcal{A}$ satisfying $\sigma \geq 0$. In fact $h$ is injective and therefore a homeomorphism. To see this, let $\sigma \geq 0$ and let $\sigma^{\prime} \leq \mathcal{C}$ be the unique face of the chamber containing $\sigma$. Then $\sigma^{\prime} \cap \mathrm{O}=\sigma$ and $\mathrm{K}_{\sigma^{\prime}}=\mathrm{K}_{\sigma}$ and the restriction of $\mathrm{h}$ to the stratum corresponding to $\sigma^{\prime}$ is the bijection

$$
\mathrm{h}=\mathrm{id} \times \exp : \mathrm{K} /\left[\mathrm{K}_{\sigma}, \mathrm{K}_{\sigma}\right] \times \sigma \rightarrow \mathrm{K} /\left[\mathrm{K}_{\sigma}, \mathrm{K}_{\sigma}\right] \times \exp \sigma .
$$

We can now show that certain rather special singularities of $\mathrm{DK}_{\mathrm{impl}}$ are removable.

4.20. Theorem. Let $\sigma$ be a face of $\mathcal{A}$ satisfying $\left[\mathrm{K}_{\sigma}, \mathrm{K}_{\sigma}\right] \cong \mathbf{S U}(2)^{\mathrm{k}}\left(\right.$ resp. $\left[\mathfrak{k}_{\sigma}, \mathfrak{k}_{\sigma}\right] \cong$ $\left.\mathfrak{s u}(2)^{\mathrm{k}}\right)$ for some $\mathrm{k}$ and possessing a vertex $\xi$ such that $\exp \xi$ is central. Then $\mathrm{DK}_{\text {impl }}$ is a smooth quasi-Hamiltonian $\mathrm{K} \times \mathrm{T}$-manifold (resp. orbifold) in a neighbourhood of the stratum corresponding to $\sigma$. 
Proof. After acting by the central element $\exp -\xi$ we may assume $\xi=0$ and $\sigma \geq 0$. Then the stratum of $D K_{i m p l}$ associated with $\sigma$ is contained in the image $(K \times \exp U)_{\text {impl }}$ of $h$. Let $\sigma^{\prime}$ be the face of the chamber containing $\sigma$. Put $X=D K_{\text {impl }}$ and $X^{\prime}=T^{*} K_{\text {impl }}$. Let $X_{\sigma}=K /\left[K_{\sigma}, K_{\sigma}\right] \times \exp \sigma$ be the stratum of $X$ associated with $\sigma$ and let $X_{\sigma}^{\prime}=K /\left[K_{\sigma}, K_{\sigma}\right] \times \sigma^{\prime}$ be the stratum of $X^{\prime}$ associated with $\sigma^{\prime}$. Let $\left(\omega_{\sigma}, \Psi_{\sigma}\right)$ denote the quasi-Hamiltonian structure on $X_{\sigma}$ and $\left(\omega_{\sigma}^{\prime}, \Psi_{\sigma}^{\prime}\right)$ the Hamiltonian structure on $X_{\sigma}^{\prime}$. It follows from Proposition 4.15 that

$$
h^{*} \Psi_{\sigma}=\exp ^{\sim} \circ \Psi_{\sigma}^{\prime} \quad \text { and } \quad \bar{f}^{*} \omega_{\sigma}=\omega_{\sigma}^{\prime}+\left(\Psi_{\sigma}^{\prime}\right)^{*} \varpi,
$$

where $\exp ^{\sim}$ is now the exponential map for $\mathrm{K} \times \mathrm{T}$. By [GJS02, Proposition 6.15] the singularities of $\mathrm{T}^{*} \mathrm{~K}_{\text {impl }}$ are removable at $X_{\sigma}^{\prime}$ in the sense that the open set $Y=\bigcup_{\tau \geq \sigma} X_{\tau}^{\prime}$ possesses a smooth structure (resp. an orbifold structure if $\left[\mathfrak{k}_{\sigma}, \mathfrak{k}_{\sigma}\right] \cong$ $\left.\mathfrak{s u}(2)^{\mathrm{k}}\right)$ and the Hamiltonian structure on the open stratum extends smoothly to this open set. With respect to this smooth structure the strata $X_{\tau}^{\prime}$ for $\tau \geq \sigma$ are smooth submanifolds of $Y$ and the Hamiltonian structure on $Y$ restricts to the given Hamiltonian structures on the strata. From 4.21 we then see that the quasiHamiltonian structure likewise extends smoothly to $\mathrm{Y}$. Via the homeomorphism $\mathrm{h}$ we now obtain a smooth quasi-Hamiltonian $\mathrm{K} \times \mathrm{T}$-manifold (resp. orbifold) structure on $h(Y)=\bigcup_{\tau \geq \sigma} X_{\tau}$.

Examples. Next we describe in more detail the imploded cross-sections of the doubles of $\mathbf{S U}(n)$ and $\mathbf{S U}(2, \mathbf{H})$. It turns out that certain strata in these spaces have a nonsingular closure. This rather serendipitous observation brings to light a family of quasi-Hamiltonian manifolds, which surprisingly turns out to be new. (See Theorem 4.26) In each of the examples below we specify an ordering $\sigma_{0}$, $\sigma_{1}, \ldots, \sigma_{\mathrm{r}}$ of the vertices of the alcove. The face corresponding to a subset I of $\{0,1, \ldots, r\}$ is then denoted by $\sigma_{I}$, the centralizer of $\sigma_{I}$ by $K_{I}$ and the associated stratum of $X=\mathrm{DK}_{\text {impl }}$ by $X_{\mathrm{I}}$.

4.22. Example. Let $\mathrm{K}=\mathbf{S U}(3)$ and let $\mathrm{T}$ be its standard maximal torus. The alcove is an open equilateral triangle (cf. Example A.18) with three vertices $\sigma_{j}$, corresponding to the elements $\mathrm{c}^{j}$ of the centre of $\mathrm{K}$. The one-dimensional faces are the open segments $\sigma_{j k}$ joining $\sigma_{j}$ to $\sigma_{k}$, where $0 \leq j<k \leq 2$.

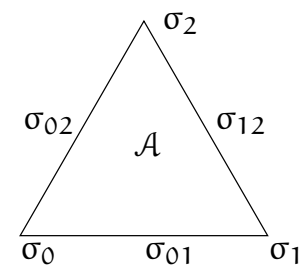

The centralizers and their commutator subgroups are

$$
\begin{array}{lll}
\mathrm{K}_{\mathcal{A}}=\mathrm{T}, & \mathrm{K}_{j k} \cong \mathbf{S}(\mathbf{U}(2) \times \mathbf{U}(1)), & \mathrm{K}_{\mathrm{j}}=\mathrm{K}, \\
{\left[\mathrm{K}_{\mathcal{A}}, \mathrm{K}_{\mathcal{A}}\right]=\{\mathrm{I}\},} & {\left[\mathrm{K}_{\mathrm{jk}}, \mathrm{K}_{\mathrm{jk}}\right] \cong \mathbf{S U}(2),} & {\left[\mathrm{K}_{j}, \mathrm{~K}_{\mathrm{j}}\right]=\mathrm{K} .}
\end{array}
$$

Thus the open stratum $X_{\mathcal{A}}$ is diffeomorphic to $\mathbf{S U}(3) \times \mathcal{A}$ and has real dimension 10; the intermediate strata $X_{j k}$ are diffeomorphic to $\mathbf{S U}(3) / \mathbf{S U}(2) \times(0,1) \cong S^{5} \times$ $(0,1)$; and the smallest strata $X_{j}$ are points in one-to-one correspondence with the centre. By Theorem 4.20 the $X_{j k}$ are removable singularities. By [GJS02, Example 
6.16] $\mathrm{T}^{*} \mathrm{~K}_{\mathrm{impl}}$ is isomorphic to the quadric cone

$$
\left\{(y, x) \in\left(\mathbf{C}^{3}\right)^{*} \times \mathbf{C}^{3} \mid y(x)=0\right\}
$$

with a Kähler structure obtained by restricting the ambient flat Kähler structure on $\left(\mathbf{C}^{3}\right)^{*} \times \mathbf{C}^{3}$. Because of the local isomorphism between $T^{*} K_{\text {impl }}$ and $D K_{\text {impl }}$ we conclude that the three smallest strata of $\mathrm{DK}_{\text {impl }}$ are genuine singularities. The intermediate strata in $T^{*} K_{\text {impl }}$ are the punctured axes, $\left\{(y, 0) \in\left(\mathbf{C}^{3}\right)^{*} \times \mathbf{C}^{3} \mid y \neq 0\right\}$ and $\left\{(0, x) \in\left(\mathbf{C}^{3}\right)^{*} \times \mathbf{C}^{3} \mid x \neq 0\right\}$. The closure of each is a copy of a flat $\mathbf{C}^{3}$. By the argument given in the proof of Theorem 4.20 we conclude that the closure of each $X_{j k}$ is nonsingular, namely a six-sphere. The upshot is that $S^{6}$ is a quasiHamiltonian SU(3)-manifold!

4.23. Example. The latter observation generalizes to $K=\mathbf{S U}(n)$. Let $T$ be the standard maximal torus and $\mathcal{A}$ the alcove given in Example $\mathrm{A} .18$ with $n$ vertices $\sigma_{j}$ corresponding to the elements $c^{j}$ of the centre of $K$. As invariant inner product on $\mathfrak{k}$ we take $(\xi, \eta)=-\left(4 \pi^{2}\right)^{-1} \operatorname{tr}(\xi \eta)$. This choice of inner product makes the embedding $\mathfrak{t} \rightarrow \mathbf{R}^{\mathfrak{n}}$ of Example A.18 an isometry, where we give $\mathfrak{t}$ the induced inner product and $\mathbf{R}^{\mathrm{n}}$ the standard inner product. Via the isomorphism $\mathfrak{t} \cong \mathfrak{t}^{*}$ given by the inner product the vertex $\sigma_{j} \in \mathfrak{t}$ is identified with the $j$-th fundamental weight $\varpi_{j} \in \mathfrak{t}^{*}$. The edge $\sigma_{01}$ of the alcove has centralizer $\mathrm{K}_{01}=\mathbf{S}(\mathbf{U}(1) \times \mathbf{U}(\mathrm{n}-1))$, so for $n>3$ the corresponding stratum $X_{01}$ in $X$ consists of genuine singularities. Nevertheless we assert that its closure $\bar{X}_{01}$ possesses the structure of a smooth quasi-Hamiltonian $\mathbf{U}(n)$-manifold. Let $\sigma_{01}^{\prime}=\left\{x \sigma_{1} \mid x>0\right\}$ be the unique edge of the chamber $\mathcal{C}=\mathcal{C}^{\vee}$ containing $\sigma_{01}$. Consider $\mathbf{C}^{\mathfrak{n}}$ with its standard Hermitian structure $\langle z, w\rangle=z^{*} w$ and its standard $\mathbf{U}(\mathrm{n})$-action. The corresponding Hamiltonian $\mathbf{U}(n)$-structure $\left(\omega_{0}, \Phi_{0}\right)$ is given by

$$
\omega_{0}=\operatorname{Im}\langle\cdot, \cdot\rangle=\frac{i}{2} \sum_{j} \mathrm{~d} z_{j} \mathrm{~d} \bar{z}_{j}, \quad \Phi_{0}(z)=-2 \pi^{2} i z z^{*},
$$

where we have used the inner product to write $\Phi$ as a map into $\mathfrak{k}$. Define $\mathcal{F}: K \times$ $\sigma_{01}^{\prime} \rightarrow C^{n}$ by $\mathcal{F}\left(k, \chi \sigma_{1}\right)=\sqrt{\chi / \pi} k_{1}$, where $k_{1} \in S^{2 n-1}$ denotes the first column of the unitary matrix $k$. The image of $\mathcal{F}$ is $C^{\mathfrak{n}}-\{0\}$. Observe that $k_{1}=k v_{1}$, where $v_{1}=(1,0, \ldots, 0)$ is the fundamental weight vector in $\mathbf{C}^{\mathfrak{n}}$, and that the stabilizer of $v_{1}$ is $\left[K_{01}, K_{01}\right] \cong \mathbf{S U}(n-1)$. Hence $\mathcal{F}$ descends to an injective map $f: X_{01}^{\prime} \rightarrow \mathbf{C}^{n}$, where $X_{01}^{\prime}=K /\left[K_{01}, K_{01}\right] \times \sigma_{01}^{\prime}$. Since $X_{01}^{\prime}$ is exactly the stratum of $X^{\prime}=T^{*} K_{\text {impl }}$ associated with the face $\sigma_{01}^{\prime}$, it follows from [GJS02, Proposition 6.8(ii)] that $f$ is a symplectic embedding. (In fact $f$ is the restriction to a single stratum of a symplectic embedding of $X^{\prime}$ into the sum of the fundamental representations.) Clearly $f$ extends to a homeomorphism $f: \bar{X}_{01}^{\prime}=X_{01}^{\prime} \cup X_{0}^{\prime} \rightarrow C^{n}$, which we regard as a chart defining a smooth structure on $\bar{X}_{01}^{\prime}$. Pulling back the symplectic structure on $C^{n}$ via $f$ we obtain a symplectic structure on $\bar{X}_{01}^{\prime}$ for which $X_{01}^{\prime}$ and $X_{0}^{\prime}$ are smooth symplectic submanifolds. The chart $\mathrm{f}$ is $\mathrm{K} \times \mathrm{T}$-equivariant with respect to the (noneffective) action $(k, t) \cdot z=k t_{1}^{-1} z$ on $C^{n}$, where $t=\operatorname{diag}\left(t_{1}, t_{2}, \ldots, t_{n}\right)$, so $\bar{X}_{01}^{\prime}$ is isomorphic to $\mathrm{C}^{\mathfrak{n}}$ as a Hamiltonian $\mathrm{K} \times \mathrm{T}$-manifold. There are similar charts on $\bar{X}_{01}=X_{01} \cup X_{0} \cup X_{1}$. We have $\mathcal{F}\left(1, \sigma_{1}\right)=\pi^{-1 / 2} v_{1}$, so precomposing $f$ with $h^{-1}$, where $h$ is the homeomorphism 4.19, we obtain a homeomorphism

$$
\phi_{0}: X_{01} \cup X_{0} \rightarrow D
$$


where $D$ is the open ball of radius $1 / \sqrt{\pi}$ in $C^{n}$. Using $\phi_{0}$ as a chart we conclude from Proposition 4.15 that $X_{01} \cup X_{0}$ is a smooth quasi-Hamiltonian $\mathrm{K} \times \mathrm{T}$-manifold isomorphic to the exponentiation of the Hamiltonian K-manifold ( $\left.D, \omega_{0}, \Phi_{0}\right)$. The inverse $\phi_{0}^{-1}: D-\{0\} \rightarrow X_{01}$ is given by

$$
z \mapsto\left(k\left[\mathrm{~K}_{01}, \mathrm{~K}_{01}\right], \exp \left(\pi\|z\|^{2} \sigma_{1}\right)\right)
$$

where $k \in K$ is any matrix whose first column is $z /\|z\|$. There is a similar chart

$$
\phi_{1}: X_{01} \cup X_{1} \rightarrow D \text {, }
$$

which is compatible with $\phi_{0}$. It is constructed by precomposing $\phi_{0}$ with a certain discrete symmetry of $X$ of the kind considered in Propositions 4.11 and 4.13 From Example A.18 we see that the action of the central element $c=e^{-2 \pi i / n} \mathrm{I}$ on $\mathcal{A}$ sends $\sigma_{j}$ to $\sigma_{j+1}$, while the longest Weyl group element $w_{0}$ sends $\sigma_{j}$ to $\sigma_{n-j}$. (Here the subscripts are taken modulo n.) Therefore the action of $c^{-1}$ followed by that of $w_{0}$ is a symmetry of the alcove that maps the edge $\sigma_{01}$ to itself, interchanging the two vertices $\sigma_{0}$ and $\sigma_{1}$. This Euclidean symmetry of $\mathcal{A}$ lifts to the diffeomorphism $\mathfrak{B}_{n_{0}} \circ \mathfrak{A}_{\widehat{c}}^{-1}: \mathrm{DK} \rightarrow \mathrm{DK}$, where $\hat{c}$ is as in Example 4.9 and $n_{0}$ as in Example 4.14 The induced map on the implosion $X=D K_{\text {impl }}$ maps $X_{01}$ to itself and interchanges $X_{0}$ and $X_{1}$. Since $\operatorname{Ad} \widehat{c}\left(\sigma_{1}\right)=-\sigma_{n-1}$ we have $\operatorname{Ad} \widehat{c}^{-1}\left(\exp \left(\chi \sigma_{1}\right)\right)=\exp \left(-\chi \sigma_{n-1}\right)$ for $0<x<1$, and so

$$
\begin{aligned}
\pi(\hat{\mathbf{c}})^{-1} \operatorname{Ad} \hat{c}^{-1}\left(\exp \left(x \sigma_{1}\right)\right)=\mathrm{c}^{-1} & \exp \left(-\chi \sigma_{n-1}\right) \\
& =\exp \sigma_{n-1} \exp \left(-\chi \sigma_{n-1}\right)=\exp \left((1-\chi) \sigma_{n-1}\right) .
\end{aligned}
$$

Therefore $\mathfrak{A}_{\widehat{c}}^{-1}\left(k, \exp \left(\chi \sigma_{1}\right)\right)=\left(k \hat{c}, \exp \left((1-\chi) \sigma_{n-1}\right)\right)$ and

$$
\mathfrak{B}_{n_{0}} \circ \mathfrak{A}_{\hat{c}}^{-1}\left(k, \exp \left(x \sigma_{1}\right)\right)=\left(k \hat{c} n_{0}^{-1}, \exp \left((1-x) \sigma_{1}\right)\right) .
$$

Hence the induced map $\psi: \bar{X}_{01} \rightarrow \bar{X}_{01}$ is given by

$$
\psi\left(\left(k, \exp \left(x \sigma_{1}\right) \bmod \sim\right)=\left(k \hat{c} n_{0}^{-1}, \exp \left((1-x) \sigma_{1}\right)\right) \bmod \sim .\right.
$$

Our second chart on $\bar{X}_{01}$ is then $\phi_{1}=\phi_{0} \circ \psi: X_{01} \cup X_{1} \rightarrow D$. An easy calculation shows that the first column of the matrix $k \hat{c} n_{0}^{-1}$ is $-k_{1}$. Using (4.24) we then find that the transition map $\phi_{1} \circ \phi_{0}^{-1}=\phi_{0} \circ \psi \circ \phi_{0}^{-1}$ is given by

$$
z \mapsto-\frac{\sqrt{\pi^{-1}-\|z\|^{2}}}{\|z\|} z .
$$

Therefore the pair of charts $\left(\phi_{0}, \phi_{1}\right)$ is a smooth atlas and $\bar{X}_{01}$ is diffeomorphic to $S^{2 \mathfrak{n}}$. By Propositions 4.11 and 4.13 the map $\mathfrak{B}_{\mathfrak{n}_{0}} \circ \mathfrak{A}_{\hat{c}}^{-1}$ reverses the two-form on DK. Consequently $\psi$ reverses the two-form on $\bar{X}_{01}$. The homomorphism $p: K \times T \rightarrow \mathbf{U}(n)$ defined by $p(k, t)=k\left(t_{1}^{-1} I_{n}\right)$ is surjective; its kernel is the kernel of the $K \times T$-action on $\bar{X}_{01}$; and the charts $\phi_{0}$ and $\phi_{1}$ are $(K \times T) / \operatorname{ker} p=\mathbf{U}(n)$ equivariant. Therefore $\bar{X}_{01}$ is in effect a quasi-Hamiltonian $\mathbf{U}(n)$-manifold. Comparing (4.25) with the transition formula (B.5) for the quasi-Hamiltonian $2 n$-sphere constructed in Appendix B we draw the following conclusion.

4.26. Theorem. The closure of the stratum $X_{01}$ of $X=D S U(n)_{i m p l}$ is a smooth quasiHamiltonian $\mathbf{U}(n)$-manifold isomorphic to the spinning $2 n$-sphere of Theorem B.10 Under this isomorphism the antipodal map on $\mathrm{S}^{2 \mathrm{n}}$ corresponds to the involution of $\overline{\mathrm{X}}_{01} o b$ tained by lifting the symmetry of the alcove $\mathcal{A}$ that reverses the edge $\sigma_{01}$. 
4.27. Example. Let $\mathrm{K}=\mathbf{U}(2, \mathbf{H})=\mathbf{S p}(2)$, the group of unitary $2 \times 2$-matrices over the quaternions $\mathbf{H}$, with maximal torus $\mathbf{T}=\left\{\operatorname{diag}\left(e^{2 \pi i x_{1}}, e^{2 \pi i x_{2}}\right)\right\} \cong \mathbf{U}(1)^{2}$. Identifying $\mathfrak{t}$ with $\mathbf{R}^{2}$ via the map $x \mapsto 2 \pi i \operatorname{diag}\left(x_{1}, x_{2}\right)$ we can write the simple roots as

$$
(2 \pi i)^{-1} \alpha_{1}(x)=x_{1}-x_{2} \quad \text { and } \quad(2 \pi i)^{-1} \alpha_{2}(x)=2 x_{2}
$$

and the minimal root as $(2 \pi i)^{-1} \alpha_{0}(x)=-2 x_{1}$. The alcove is the isosceles right triangle $1 / 2>x_{1}>x_{2}>0$. There are two vertices, $\sigma_{0}$ resp. $\sigma_{1}$, corresponding to the central elements I resp. $-\mathrm{I}$, and a third vertex $\sigma_{2}$ corresponding to the element $\operatorname{diag}(-1,1)$. The edge $\sigma_{01}$ is the hypotenuse and the edges $\sigma_{02}$ and $\sigma_{12}$ are the right edges. Under the exponential map these correspond to elements of the torus of the form $\operatorname{diag}(t, t), \operatorname{diag}(t, 1)$ and $\operatorname{diag}(-1, t)$, respectively.

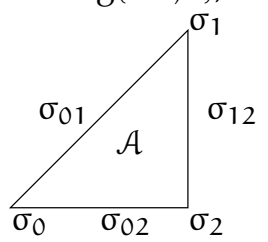

The centralizers and their commutator subgroups are as follows.

\begin{tabular}{|l|l|l|}
\hline$\sigma$ & $\mathrm{K}_{\sigma}$ & {$\left[\mathrm{K}_{\sigma}, \mathrm{K}_{\sigma}\right]$} \\
\hline \hline $\mathcal{A}$ & $\mathrm{T}$ & 1 \\
01 & $\mathbf{U}(2)$ & $\mathbf{S U}(2)$ \\
02 & $\mathbf{U}(1) \times \mathbf{U}(1, \mathbf{H})$ & $1 \times \mathbf{U}(1, \mathbf{H})$ \\
12 & $\mathbf{U}(1, \mathbf{H}) \times \mathbf{U}(1)$ & $\mathbf{U}(1, \mathbf{H}) \times 1$ \\
0 or 1 & $\mathrm{~K}$ & $\mathrm{~K}$ \\
2 & $\mathbf{U}(1, \mathbf{H}) \times \mathbf{U}(1, \mathbf{H})$ & $\mathbf{U}(1, \mathbf{H}) \times \mathbf{U}(1, \mathbf{H})$ \\
\hline
\end{tabular}

Here $\mathbf{U}(2)$ is the collection of matrices in $\mathrm{K}$ with complex coefficients, $\mathbf{U}(1) \times$ $\mathbf{U}(1, \mathbf{H})$ is the collection of matrices of the form $\operatorname{diag}(a, b)$ with $a \in \mathbf{C}, b \in \mathbf{H}$ and $|\mathrm{a}|=|\mathrm{b}|=1$, etc. The alcove corresponds to a twelve-dimensional stratum $\mathbf{U}(2, \mathbf{H}) \times \mathcal{A}$. The strata corresponding to all three edges are eight-dimensional and by Theorem 4.20 are removable singularities. The strata $X_{0}$ and $X_{1}$ are genuine singularities. The stratum $X_{2}$ is diffeomorphic to $K / K_{2}=\mathbf{H P} \mathbf{P}^{1} \cong S^{4}$. Note that $\mathrm{K}_{2}$ is semisimple and is not the centralizer of any element in the Lie algebra of $\mathrm{K}$. We do not know of any relationship between the quasi-Hamiltonian structure of $S^{4}$ considered as an $\mathbf{U}(2, \mathbf{H})$-conjugacy class and the $\mathbf{S U}(2) \times \mathbf{U}(1)$-structure of Theorem 4.26 As in Example 4.23 it can be shown that $\bar{X}_{02}$ and $\bar{X}_{12}$ are smooth quasi-Hamiltonian $\mathbf{U}(2, \mathbf{H})$-manifolds diffeomorphic to $\mathbf{H P}^{2}$.

\section{IMPLOSION AND WEIGHTED PROJECTIVE SPACES}

The Hamiltonian case. The construction of an imploded cross-section as a subquotient of a smooth manifold is satisfying in some respects, but less so in others. In this section we give a different description of the universal imploded crosssection $\mathrm{DK}_{\mathrm{impl}}$, namely as the orbit under $\mathrm{K}$ of a toric variety embedded in a representation of K. In [GJS02, Section 6] a similar construction is performed in the Hamiltonian case, which we review first.

Let $\mathrm{G}=\mathrm{K}^{\mathrm{C}}$ be the complexification of $\mathrm{K}$ and let $\mathrm{N}$ be the maximal unipotent subgroup of $G$ with Lie algebra $\mathfrak{n}=\bigoplus_{\alpha \in R_{+}} \mathfrak{g}_{\alpha}$ (where $R_{+}$denotes the set of positive roots). Let $\varpi_{1}, \ldots, \varpi_{r}$ be the fundamental weights of $G$, let $V_{p}$ denote the 
irreducible G-module with highest weight $\varpi_{p}$, and define $E=\bigoplus_{p=1}^{r} V_{p}$. Let $E^{N}$ be the subspace of $\mathrm{N}$-invariants; this is

$$
\mathrm{E}^{\mathrm{N}}=\bigoplus_{\mathrm{p}=1}^{\mathrm{r}} \mathrm{C} v_{\mathrm{p}},
$$

where $v_{p} \in V_{p}$ is a highest-weight vector. The subset $G_{N}=G^{N}$ of $E$ consists of finitely many G-orbits and is a closed affine subvariety of $E$ (because it is equal to the closure of the G-orbit through $v_{1}+v_{2}+\cdots+v_{r}$ ). The module $E$ has a unique K-invariant Hermitian inner product such that each $v_{p}$ has length 1 ; and we equip the orbits in $G_{N}$ with the Kähler structures induced by this flat Kähler structure on E. In [GJS02, Section 6] is constructed a $\mathrm{K} \times \mathrm{T}$-equivariant homeomorphism $\mathrm{T}^{*} \mathrm{~K}_{\text {impl }} \rightarrow \mathrm{G}_{\mathrm{N}}$, which maps each stratum in $\mathrm{T}^{*} \mathrm{~K}_{\text {impl }}$ symplectomorphically onto a G-orbit in $G_{N}$. This allows us to view $T^{*} K_{\text {impl }}$ as a complex algebraic variety with a Kähler structure on each stratum. Observe also that $G_{N}=K E^{N}$ because of the Iwasawa decomposition $G=K A N$ (with $A=\exp i t$ ). In addition, $E^{N}$ with its natural T-action and moment map $\Phi\left(\sum_{p} z_{p} v_{p}\right)=\Sigma_{p}\left|z_{p}\right|^{2} \Phi_{p}$ is the symplectic toric variety associated with the polyhedron $\overline{\mathrm{e}}$. (See [GJS02, Remark 6.7], but note the different normalization of the moment map.)

It is clear from the examples in Section 4 that the quasi-Hamiltonian imploded cross-section $\mathrm{DK}_{\mathrm{impl}}$ is not a complex algebraic variety. (Indeed $\mathrm{DK}_{\mathrm{impl}}=\mathrm{S}^{4}$ for $\mathrm{K}=\mathbf{S U}(2)$.) Nevertheless it turns out that we can still build $\mathrm{DK}_{\text {impl }}$ as the K-orbit of a toric variety embedded in a K-module.

Construction of a toric variety. To adapt the construction of $G_{N}$ to the quasiHamiltonian category, we start by replacing the chamber $\mathcal{C}$ by the alcove $\mathcal{A}$ and building a toric variety $X$ corresponding to it. (We use the isomorphism $t \rightarrow t^{*}$ given by the inner product to view $\mathcal{A}$ as a subset of $t^{*}$.) We may assume without loss of generality that $K$ is almost simple. Then the alcove is a simplex, so the toric variety is just a weighted projective space and can be characterized as a symplectic cut of the T-module $E^{N}$ considered in 5.1. Let $\alpha_{1}, \ldots, \alpha_{r}$ be the simple roots of $K$ and $\alpha_{r+1}$ the highest root. Let $H$ be the circle subgroup of $T$ generated by $2 \pi i \alpha_{r+1}^{\vee}$ and let $X$ be the symplectic cut (in the sense of [Ler95]) of $E^{N}$ relative to $H$ at level $k=2 /\left\langle\alpha_{r+1}, \alpha_{r+1}\right\rangle$. (Modulo the identification $\mathfrak{t} \cong \mathfrak{t}^{*}$ we have $\alpha_{r+1}^{\vee}=$ $2 \alpha_{r+1} /\left\langle\alpha_{r+1}, \alpha_{r+1}\right\rangle$, so $\alpha_{r+1}^{\vee}=k \alpha_{r+1}$.) Then $X$ is a symplectic orbifold which inherits a Hamiltonian $\mathrm{T}$-action from $\mathrm{E}^{\mathrm{N}}$ with a moment map which we shall also denote by $\Phi$. The moment polytope of $X$ is obtained by truncating the moment polytope of $E^{N}$ at the hyperplane $\lambda\left(\alpha_{r+1}^{\vee}\right)=k$, i.e.

$$
\begin{aligned}
\Phi(\mathrm{X}) & =\Phi\left(\mathrm{E}^{\mathrm{N}}\right) \cap\left\{\lambda \in \mathfrak{t}^{*} \mid(2 \pi \mathfrak{i})^{-1} \lambda\left(\alpha_{\mathrm{r}+1}^{\vee}\right) \leq \mathrm{k}\right\} \\
& =\overline{\mathcal{C}} \cap\left\{\lambda \in \mathfrak{t}^{*} \mid(2 \pi \mathfrak{i})^{-1}\left\langle\lambda, \alpha_{\mathrm{r}+1}\right\rangle \leq 1\right\} \\
& =\overline{\mathcal{A}} .
\end{aligned}
$$

Note that the size of the image of $\mathcal{A}$ in $\mathrm{t}^{*}$, and hence the symplectic structure on $\mathrm{X}$, depend on the choice of the inner product on $\mathfrak{k}$. The T-action on $E^{N}$ is effective and therefore so is the induced $T$-action on $X$. We conclude that $X$ is a symplectic toric orbifold in the sense of [LT97]. By [loc. cit., Theorems 7.4, 8.1] such orbifolds are classified by labelled polytopes, simple rational polytopes in $t^{*}$ with a label attached to each face of codimension one. The label is the integer $l$ such that every element 
in the preimage of the face under the moment map has orbifold structure group $\mathbf{Z} / \mathbf{l Z}$. We can now describe $X$ more explicitly as follows. The coefficients $m_{1}, \ldots$, $m_{r}$ in the expansion of the coroot of the highest root,

$$
\alpha_{r+1}^{\vee}=\sum_{p=1}^{r} m_{p} \alpha_{p}^{\vee},
$$

are relatively prime positive integers. Set $m_{r+1}=1$ and $m=\left(m_{1}, \ldots, m_{r+1}\right)$.

\subsection{Lemma. $\quad$ (i) $\mathrm{X} \cong \mathbf{C P}_{\mathrm{m}}^{\mathrm{r}}$, the weighted projective $\mathrm{r}$-space with weights $\mathrm{m}$.}

(ii) $\mathrm{X}$ is the symplectic toric orbifold associated with the polytope $\overline{\mathcal{A}}$ with label 1 attached to every codimension-one face.

(iii) Let $\mathrm{x} \in \mathrm{X}$ and $\sigma \leq \mathcal{A}$. Suppose $\Phi(\mathrm{x}) \in \sigma$. Then $\mathrm{T}_{\mathrm{x}}=\mathrm{T} \cap\left[\mathrm{K}_{\sigma}, \mathrm{K}_{\sigma}\right]$.

Proof. The symplectic cut $X$ is by definition the symplectic quotient of $E^{N} \times C$ with respect to $\mathrm{H}$ at level $\mathrm{k}$. Here the $\mathrm{H}$-action on $\mathrm{E}^{\mathrm{N}}$ is the restriction of the T-action and the $\mathrm{H}$-action on $\mathrm{C}$ is defined infinitesimally by $\alpha_{\mathrm{r}+1}^{\vee} \cdot w=w$. Introducing coordinates $\left(z_{1}, \ldots, z_{\mathrm{r}}, z_{\mathrm{r}+1}\right)$ on $\mathrm{E} \times \mathbf{C}$ (relative to the basis $v_{1}, \ldots, v_{\mathrm{r}}$ of $\mathrm{E}$ ) and writing $h=\exp \left(t \alpha_{r+1}^{\vee}\right)$ we have

$$
h \cdot v=\left(e^{2 \pi i t \Phi_{1}\left(\alpha_{r+1}^{\vee}\right)} z_{1}, \ldots, e^{2 \pi i t \varpi_{r}\left(\alpha_{r+1}^{\vee}\right)} z_{r}\right)=\left(e^{2 \pi i m_{1} t} z_{1}, \ldots, e^{2 \pi i m_{r} t} z_{r}\right),
$$

since $\varpi_{q}\left(\alpha_{p}^{\vee}\right)=\delta_{p q}$. Thus $X$ is the symplectic quotient of $C^{r+1}$ by the $\mathbf{U}(1)$ action with weights $m$, which proves (i). Moreover, by (5.2) the T-moment map image of $X$ is the rational simplex $\overline{\mathcal{A}}$. To calculate the label attached to a face $\sigma_{p}$, note that for $p \leq r$ the T-moment map $\Phi$ maps (a suitable multiple of) $x_{p}=$ $\left(\sum_{q \neq p} v_{q}, 1\right)$ into $\sigma_{p}$. The H-stabilizer of $x_{p}$ is trivial because $\mathrm{H}$ acts with weight 1 on C. Thus $\sigma_{p}$ has label 1 for $p \leq r$. Moreover, (a suitable multiple of) the point $\left(\sum_{p=1}^{r} v_{p}, 0\right)$ is mapped into $\sigma_{r+1}$ and this point also has trivial $\mathrm{H}$-stabilizer because $\operatorname{gcd}\left(m_{1}, \ldots, m_{r}\right)=1$. Thus $\sigma_{r+1}$ has label 1. Finally, by [LT97, Lemma 6.6] the stabilizer $T_{x}$ for $x \in \Phi^{-1}(\sigma)$ is the torus whose Lie algebra is the subspace of $\mathfrak{t}$ spanned by all $\alpha^{\vee}$ that are perpendicular to $\sigma$. This shows that $\mathfrak{t}_{x}=\mathfrak{t} \cap\left[\mathfrak{k}_{\sigma}, \mathfrak{k}_{\sigma}\right]$. Now $T \cap\left[K_{\sigma}, K_{\sigma}\right]$ is a maximal torus of $\left[K_{\sigma}, K_{\sigma}\right]$. In particular it is connected and therefore $T_{x}=T \cap\left[K_{\sigma}, K_{\sigma}\right]$.

5.4. Example. Using [Bou82 Ch. 6, Planches I-IX] one easily calculates the following table.

\begin{tabular}{|l|c|}
\hline $\mathrm{K}$ & $\mathrm{m}$ \\
\hline \hline $\mathbf{A}_{\mathrm{r}}$ & $(1,1, \ldots, 1)$ \\
$\mathbf{B}_{\mathrm{r}}$ & $(1,2,2, \ldots, 2,1,1)$ \\
$\mathbf{C}_{\mathrm{r}}$ & $(1,1, \ldots, 1)$ \\
$\mathbf{D}_{\mathrm{r}}$ & $(1,2,2, \ldots, 2,1,1,1)$ \\
$\mathbf{E}_{6}$ & $(1,2,2,3,2,1,1)$ \\
$\mathbf{E}_{7}$ & $(2,2,3,4,3,2,1,1)$ \\
$\mathbf{E}_{8}$ & $(2,3,4,6,5,4,3,2,1)$ \\
$\mathbf{F}_{4}$ & $(2,3,2,1,1)$ \\
$\mathbf{G}_{2}$ & $(1,2,1)$ \\
\hline
\end{tabular}

The upshot is that $X$ is the standard projective space $\mathbf{C P}^{r}$ for $K=\mathbf{S U}(r+1)$ or $\mathrm{K}=\mathbf{U}(\mathrm{r}, \mathbf{H})$ and a weighted projective space in all other cases. 
Embedding. We want to embed $X$ into a $K$-module $V$ in such a way that the points in $\Phi^{-1}(\sigma)$ have stabilizer $\left[K_{\sigma}, K_{\sigma}\right]$; this will then give us an identification $D K_{i m p l} \cong$ $\mathrm{KX}$. For each $\sigma \leq \mathcal{A}$ one should choose a representation $W_{\sigma}$ and a point $p_{\sigma} \in W_{\sigma}$ whose stabilizer is $\left[\mathrm{K}_{\sigma}, \mathrm{K}_{\sigma}\right]$.

For the vertices, one can use the fact that $K_{\sigma}=\left[K_{\sigma}, K_{\sigma}\right]$ : one then just needs to take a faithful representation of the group, which embeds the group into a vector space $V$, and have $K$ act by the adjoint representation.

For the edges, we want, for each pair of roots $\alpha_{i}, \alpha_{j}$ generating the faces of the alcove, a representation $V_{i, j}$ and a point $p_{i, j}$ such that the stabilizer $\left[K_{i, j}, K_{i, j}\right]$ of $p_{i, j}$ is generated by the root spaces $e_{\alpha_{k}}, f_{\alpha_{k}}$, for $k \neq i, j$, and such that the torus $T$ acts on the line generated by $p_{i, j}$ by the weight $l_{j} \varpi_{j}-l_{i} \varpi_{i}$. Here we define $l_{i}=m / m_{i}$, with $m=1 \mathrm{~cm}\left(m_{1}, \ldots, m_{r+1}\right)$.

A first case is when either $i$ or $j$, say $i$, is $r+1$. One then just takes the $l_{j}$-th power of the $j$-th fundamental representation, and $p_{i, j}$ the highest weight vector.

Suppose now that $i$ and $j$ both correspond to simple roots. Then

$$
\left\langle l_{j} \varpi_{j}-l_{i} \varpi_{i}, \alpha_{k}^{\vee}\right\rangle \begin{cases}>0 & \text { for } k=j \\ <0 & \text { for } k=i \\ =0 & \text { otherwise. }\end{cases}
$$

(For the lowest root, this uses the definition of the $l_{i}$.) We can use the Weyl group to bring $l_{j} \varpi_{j}-l_{i} \varpi_{i}$ into the chamber $\mathcal{C}$, and the roots $\alpha_{k}$ for $k \neq i$ into the set of positive roots. Because of (5.5), the representation with highest weight $l_{j} \varpi_{j}-l_{i} \varpi_{i}$ will then be a multiple of one of the highest weight representations.

Now to the embedding. We proceed in an inductive fashion on the faces of the alcove, starting with the vertices. Let $\left(a_{1}, \ldots, a_{r+1}\right)$ denote the projective coordinates of a point in the weighted projective space $X$, where we can assume that $\left(a_{1}, \ldots, a_{r+1}\right)$ is of norm one.

The vertices correspond to points where only one $a_{i}$ is non-zero, and we map this to $W_{i, i}$, the representation corresponding to the vertex, by

$$
a_{i} \mapsto\left(\bar{a}_{i}\right)^{l_{i}}\left(a_{i}\right)^{l_{i}} p_{i, i}
$$

Now for the edges. For each pair $(i, j)$ with $1 \leq i, j \leq r+1$ consider the $(i, j)$-th edge $\sigma=\sigma_{i, j}$, and choose a representation $W_{i, j}$ and a point $p_{i, j}$ with stabilizer $\left[K_{\sigma_{i, j}}, K_{\sigma_{i, j}}\right]$. (Clearly we may assume that $W_{i, j}=W_{j, i}$.) We map the edge by

$$
\left(a_{i}, a_{j}\right) \mapsto\left(\bar{a}_{i}\right)^{l_{i}}\left(a_{j}\right)^{l_{j}} p_{i, j} .
$$

This is sufficient by Proposition A.5 ii). In other words, we extend this map to a map from $X$ to $V=\bigoplus_{i, j=1}^{r+1} W_{i, j}$ by

$$
\left(a_{1}, \ldots, a_{r+1}\right) \mapsto \bigoplus_{i, j=1}^{r+1}\left(\bar{a}_{i}\right)^{l_{i}}\left(a_{j}\right)^{l_{j}} p_{i, j} .
$$

(Note that for $i \neq j$ the summand $W_{i, j}=W_{j, i}$ occurs twice in $V$. ) We then have the desired embedding of $X$, and a homeomorphism $D K_{\text {impl }} \cong K X$.

5.6. Example. For $\mathbf{S U}(\mathrm{r}+1)$ we can proceed slightly differently and embed $\mathrm{X}=$ $\mathbf{C P}^{r}$ into a sum of weight spaces as follows. Consider the unit sphere

$$
\left\{\left.\left(a_{1}, \ldots, a_{r+1}\right)|| a_{1}\right|^{2}+\cdots+\left|a_{r+1}\right|^{2}=1\right\}
$$


in $\mathrm{C}^{\mathrm{r}+1}$. As the vertices of the alcove all correspond to central elements, we can set $W_{i, i}=\mathbf{C}, p_{i, i}=1$. For the edges, if $e_{1}, \ldots, e_{r+1}$ denotes the standard basis of $C^{r+1}$, we set $p_{i, j}=e_{i+1} \wedge \cdots \wedge e_{j}$ for $i<j$, and $E_{i, j}=e_{i+1} \wedge \cdots \wedge e_{r+1} \wedge e_{1} \wedge \cdots \wedge e_{j}$ for $i>j$, where these elements live in the representations $W_{i, j}=\Lambda^{j-i} C^{r+1}$ for $i \leq j$, and $W_{i, j}=\Lambda^{r+1+j-i} C^{r+1}$ for $i>j$. Set $V_{i, j}=C E_{i, j}$. Map the unit sphere to $\mathrm{U}=\bigoplus \mathrm{V}_{i, j}$ by

$$
\left(c_{1}, \ldots, c_{r+1}\right) \mapsto \sum_{i, j=1}^{r+1} \bar{c}_{i} c_{j} E_{i, j} .
$$

This map is T-equivariant, and factors through $X$. We set $W=\bigoplus_{i, j=1}^{r+1} W_{i, j}$. The orbit $\mathbf{S U}(r+1) \cdot X$ is then our universal imploded cross-section. If $\left(a_{1}, \ldots, a_{r+1}\right)$ are the coordinates of a point $p$ in $X$, with $a_{i_{1}}, a_{i_{2}}, \ldots, a_{i_{s}}$ the non-zero coordinates (with $\left.i_{1}<i_{2}<\cdots<i_{s}\right)$, the stabilizer of $p$ is $\mathbf{S U}\left(r+1+i_{1}-i_{s}\right) \times \mathbf{S U}\left(i_{2}-i_{1}\right) \times$ $\cdots \times \mathbf{S U}\left(i_{s}-i_{s-1}\right)$. This is the commutator subgroup of the stabilizer under the adjoint action of elements

$$
\operatorname{diag}\left(l_{1}, \ldots, l_{1}, l_{2}, \ldots, l_{2}, l_{3}, \ldots, l_{3}, \ldots, l_{s-1}, l_{s}, \ldots, l_{s}\right),
$$

where $l_{1}$ is repeated $r+1+i_{1}-i_{s}$ times, and the other $l_{j}$ are repeated $i_{j}-i_{j-1}$ times.

\section{THE MASTER MODULI SPACE}

Let $\Sigma$ be a compact oriented two-manifold of genus $g$ with boundary components $V_{1}, V_{2}, \ldots, V_{n}$. As before, let $K$ be a compact simply connected Lie group and choose an n-tuple $C=\left(C_{1}, C_{2}, \ldots, C_{n}\right)$ of conjugacy classes in $K$. Consider homomorphisms $\pi_{1}(\Sigma) \rightarrow K$ which map the cycle $\left[V_{i}\right]$ into the class $C_{i}$ for $1 \leq i \leq n$. The space $M(\Sigma, C)$ of all such homomorphisms modulo conjugation by $K$ can be interpreted as the space of flat connections on the trivial principal bundle $\Sigma \times \mathrm{K}$ with prescribed holonomy $c_{i}$ around $V_{i}$, modulo gauge equivalence. Atiyah and Bott [AB83 proved that $M(\Sigma, C)$ carries a natural symplectic structure on a dense open subset. Alekseev et al. [AMM98, Theorem 9.2] proved that, for $n \geq 1, M(\Sigma, C)$ is a quasi-symplectic quotient,

$$
M(\Sigma, C) \cong M(\Sigma) / / c^{n} .
$$

$\left(\right.$ On the right we reduce at the unique point $\mathrm{c}$ where $\mathrm{C}$ intersects $(\exp \overline{\mathcal{A}})^{n}$.) Here $M(\Sigma)$ is the quasi-Hamiltonian $\mathrm{K}^{\mathrm{n}}$-manifold

$$
\mathrm{M}(\Sigma)=\mathrm{DK}^{\circledast \mathrm{g}} \circledast \mathrm{DK} \mathrm{K}^{\circledast(\mathrm{n}-1)} ;
$$

DK denotes the quasi-Hamiltonian K-manifold obtained from DK by fusing the two $\mathrm{K}$-actions, and $X^{\circledast l}$ denotes the l-fold fusion product of a space $X$ with itself. Thus $M(\Sigma)=\mathrm{K}^{2(\mathrm{~g}+\mathrm{n}-1)}$ as a manifold. The isomorphism 6.1 implies, by Theorem 2.9. that $M(\Sigma, C)$ is a stratified space with symplectic strata, thus confirming the Atiyah-Bott theorem. The definition of the isomorphism involves a choice of base points $p_{i} \in V_{i}$ for $1 \leq i \leq n$, which enables one to identify $M(\Sigma)$ with the space of flat connections on $\Sigma \times \mathrm{K}$ modulo gauge transformations that are the identity at the $p_{i}$. (See AMM98, Theorems 9.1,9.3].)

The first goal of this section is to build a master moduli space $M$, which is symplectic and from which all moduli spaces $M(\Sigma, C)$ can be obtained by symplectic reduction with respect to a compact torus. Given the results of Section 3 this is 
entirely straightforward: we simply define $M=M(\Sigma)_{\text {impl }}$, the implosion of $M(\Sigma)$ with respect to $K^{n}$. This carries an action of the rn-dimensional torus $T^{n}$ (where $r$ is the rank of $\mathrm{K}$ ) and an imploded moment map $\Phi: M \rightarrow \mathrm{T}^{\mathrm{n}}$ taking values in $(\exp \overline{\mathcal{A}})^{n}$. Theorem 3.17 and Addendum 3.18 immediately imply the following result.

6.3. Theorem. The master moduli space $\mathrm{M}=\mathrm{M}(\Sigma)_{\text {impl }}$ has a $\mathrm{T}^{\mathrm{n}}$-invariant locally finite decomposition into symplectic manifolds. The $\mathrm{T}^{\mathrm{n}}$-action on each stratum is quasiHamiltonian with moment map given by the restriction of $\Phi$. Let $\mathrm{C}$ be a conjugacy class in $\mathrm{K}^{\mathrm{n}}$ and $\mathrm{c}$ its unique intersection point with $(\exp \overline{\mathcal{A}})^{\mathrm{n}}$. Then $\mathrm{M} / / \mathrm{c} \mathrm{T}^{\mathrm{n}} \cong \mathrm{M}(\Sigma, \mathrm{C})$.

Let $\Sigma^{\prime}$ be the closed Riemann surface of genus $g$ obtained by capping off the boundary components of $\Sigma$ and choose a complex structure on $\Sigma^{\prime}$. A well-known theorem due to Mehta and Seshadri [MS80] establishes an equivalence between the moduli space $M(\Sigma, C)$ and the moduli space of holomorphic vector bundles on $\Sigma^{\prime}$ with prescribed parabolic structures at the points $p_{1}, p_{2}, \ldots, p_{n}$. In $|\mathrm{HJS}|$ we will prove, for $K=\mathbf{S U}(n)$, an analogue of the Mehta-Seshadri theorem by showing that the master moduli space $M$ possesses a complex algebraic structure such that every moduli space of parabolic bundles can be written as a GIT quotient of $M$ with respect to the complex torus $\left(\mathrm{T}^{\mathrm{C}}\right)^{\mathrm{n}}$ (and we will indicate a possible approach for arbitary K).

We now want to give a more explicit description of $M$ and also draw a parallel between the various moduli spaces associated to $\Sigma$ on one hand and the following diagram of spaces on the other hand:

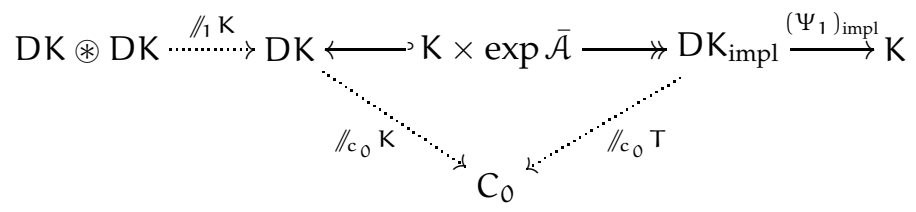

Here the dotted arrows indicate quasi-Hamiltonian reduction with respect to the relevant group, $\mathrm{C}_{0}$ denotes a conjugacy class of $\mathrm{K}$ and $\mathrm{c}_{0}$ its unique intersection point with $\exp \overline{\mathcal{A}}, \mathrm{K} \times \exp \overline{\mathcal{A}}$ is the inverse image of $\exp \overline{\mathcal{A}}$ in $\mathrm{DK}$, and $\mathrm{K} \times \exp \overline{\mathcal{A}} \rightarrow$ $\mathrm{DK}_{\text {impl }}$ is the quotient map for the equivalence relation $\sim$. The moduli spaces analogous to DK, $\mathrm{DK}_{\mathrm{impl}}$ and $\mathrm{C}_{0}$ are $M(\Sigma), M=M(\Sigma)_{\text {impl }}$ and $M(\Sigma, C)$, respectively. To find the missing spaces, we punch an extra hole with boundary circle $V_{n+1}$ in $\Sigma$ to obtain a new Riemann surface $\hat{\Sigma}$. Because of (6.2) the moduli space of $\hat{\Sigma}$ is then the quasi-Hamiltonian $\mathrm{K}^{\mathrm{n}+1}$-manifold

$$
\mathrm{M}(\hat{\Sigma})=\mathbf{D} \mathrm{K}^{\circledast \mathrm{g}} \circledast \mathrm{D} \mathrm{K}^{\circledast \mathrm{n}},
$$

and $M(\Sigma)=M(\hat{\Sigma}) / / 1 \mathrm{~K}$ by Lemma4.3i). (Indeed, if for $\mathrm{n}=0$ we define $\mathrm{M}(\Sigma)=$ $M(\hat{\Sigma}) / / 1 \mathrm{~K}$, then 6.1 is true even for $n=0$.) Thus $M(\hat{\Sigma})$ is the moduli space analogue of the space DK $\circledast$ DK on the left in [6.4. According to [AMM98 $\S 9.2$ ], in suitable coordinates $(a, b, u, v) \in \mathrm{K}^{g} \times \mathrm{K}^{g} \times \mathrm{K}^{\mathrm{n}} \times \mathrm{K}^{\mathrm{n}}$ on $\mathrm{M}(\hat{\Sigma})$ the action of $\left(k_{1}, k_{2}, \ldots, k_{n+1}\right) \in K^{n+1}$ is given by

$$
\begin{aligned}
a_{h} & \mapsto \operatorname{Ad}\left(k_{n+1}\right) a_{h}, & b_{h} & \mapsto \operatorname{Ad}\left(k_{n+1}\right) b_{h}, \\
u_{m} & \mapsto k_{n+1} u_{m} k_{m}^{-1}, & v_{m} & \mapsto \operatorname{Ad}\left(k_{m}\right) v_{m},
\end{aligned}
$$


and the moment map $\Phi=\left(\Phi_{1}, \Phi_{2}, \ldots, \Phi_{n+1}\right)$ by

$$
\begin{aligned}
\Phi_{i}(a, b, u, v) & =v_{i} \quad \text { for } i=1,2, \ldots, n, \\
\Phi_{n+1}(a, b, u, v) & =\prod_{h=1}^{g}\left[a_{h}, b_{h}\right] \prod_{m=1}^{n} \operatorname{Ad}\left(u_{m}\right) v_{m}^{-1} .
\end{aligned}
$$

The $(a, b, u, v)$ are to be thought of as holonomies along paths $A_{h}, B_{h}(1 \leq h \leq g)$ and $U_{m}, V_{m}(1 \leq m \leq n)$. The $A_{h}$ and $B_{h}$ originate at a base point $p_{n+1}$ on the boundary component $V_{n+1}$ of $\hat{\Sigma}$ and represent the standard homology basis of the closed surface $\Sigma^{\prime}$, and the $U_{m}$ are paths joining $p_{n+1}$ to $p_{m}$, the base points on the boundary components $V_{m}$ of $\Sigma$. We can further assume that these paths do not intersect except at their endpoints, and are such that we can write $\Sigma$ in the usual way as the quotient of a polygon with sides (in order)

$$
\begin{aligned}
A_{1}, B_{1}, A_{1}^{-1}, B_{1}^{-1}, A_{2}, B_{2}, A_{2}^{-1}, B_{2}^{-1}, \ldots, A_{g}, B_{g}, A_{g}^{-1}, B_{g}^{-1}, \\
\\
u_{1}, v_{1}^{-1}, u_{1}^{-1}, u_{2}, v_{2}^{-1}, u_{2}^{-1}, \ldots, u_{n}, v_{n}^{-1}, u_{n}^{-1},
\end{aligned}
$$

with identifications as given along the edges. The fundamental group of $\Sigma$ is then the group generated by $A_{h}, B_{h}, U_{m} V_{m}^{-1} U_{m}^{-1}$ subject to the relation

$$
\prod_{h=1}^{g}\left[A_{h}, B_{h}\right] \prod_{m=1}^{n} u_{m} V_{m}^{-1} u_{m}^{-1}=1 \text {. }
$$

Using the coordinate system $(a, b, u, v)$ we can describe $M(\Sigma)$ as

$$
M(\Sigma)=M(\hat{\Sigma}) / / 1 \mathrm{~K}=\left\{(\mathrm{a}, \mathrm{b}, \mathrm{u}, v) \in \mathrm{K}^{2(\mathrm{~g}+\mathrm{n})} \mid \Phi_{\mathrm{n}+1}(\mathrm{a}, \mathrm{b}, \mathrm{u}, v)=1\right\} / K,
$$

where $K$ acts as the last factor of $K^{n+1}$. The preimage of $(\exp \overline{\mathcal{A}})^{n}$ under the moment map for the residual $\mathrm{K}^{\mathrm{n}}$-action is then

$$
\mathrm{M}(\Sigma, \mathcal{A})=\left\{(\mathrm{a}, \mathrm{b}, \mathrm{u}, v) \in \mathrm{K}^{2(\mathrm{~g}+\mathrm{n})} \mid \Phi_{\mathrm{n}+1}(\mathrm{a}, \mathrm{b}, \mathrm{u}, \boldsymbol{v})=1, \quad v \in(\exp \overline{\mathcal{A}})^{\mathrm{n}}\right\} / \mathrm{K} .
$$

This is to be viewed as the analogue of the space $K \times \exp \overline{\mathcal{A}}$ in 6.4 and can be interpreted as the moduli space of flat connections whose holonomy around the loops $\mathrm{V}_{i}$ is restricted to lie in $\exp \overline{\mathcal{A}}$. Implosion gives

$$
M=M(\Sigma)_{\text {impl }}=\left\{(a, b, u, v) \in K^{2 g} \times\left(D K_{i m p l}\right)^{n} \mid \Phi_{n+1}(a, b, u, v)=1\right\} / K,
$$

where $\left(u_{\mathrm{m}}, v_{\mathrm{m}}\right) \in \mathrm{K} \times \exp \overline{\mathcal{A}}$ represents an element of $\mathrm{DK} \mathrm{K}_{\mathrm{impl}}$. From 6.5 we see that the moment map $M \rightarrow T^{n}$ is the map induced by the holonomy map $(\mathrm{a}, \mathrm{b}, \mathrm{u}, v) \mapsto v$. Reduction at $\mathrm{c} \in(\exp \overline{\mathcal{A}})^{\mathrm{n}}$ gives

$$
M(\Sigma, C)=\left\{(a, b, u, c) \in K^{2 g} \times\left(D K_{i m p l} / T\right)^{n} \mid \Phi_{n+1}(a, b, u, c)=1\right\} / K,
$$

where $\left(u_{m}, c_{m}\right) \in K \times \exp \overline{\mathcal{A}}$ represents an element of $D K_{i m p l} / T$. Finally, setting $u=1$ in 6.6 gives the space of all representations of the fundamental group,

$$
\operatorname{Hom}\left(\pi_{1}(\Sigma), K\right) / K=\left\{(a, b, v) \in K^{2 g+n} \mid \Phi_{n+1}(a, b, 1, v)=1\right\} / K,
$$

and a natural map $f: M(\Sigma) \rightarrow \operatorname{Hom}\left(\pi_{1}(\Sigma), K\right) / K$, which descends to a map $f_{\text {impl }}$ from $M(\Sigma)_{\text {impl }}$ to $\operatorname{Hom}\left(\pi_{1}(\Sigma), K\right) / K$. The moduli space counterpart of diagram 
(6.4) is thus

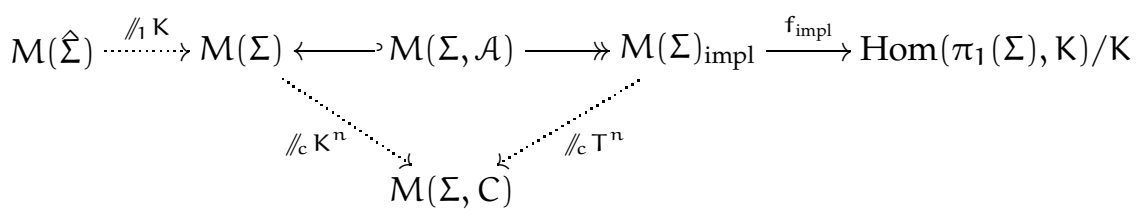

We do not know if $f$ or $f_{\text {impl }}$ can be interpreted as a moment map in any sense.

\section{Appendix A. THE CONJUGATION ACTION}

This appendix is a review of some facts concerning the conjugation, or adjoint, action of a compact Lie group on itself. Several of these facts are well-known, but for lack of a convenient reference we provide short proofs. Let $\mathrm{K}$ be a simply connected compact Lie group with Lie algebra $\mathfrak{k}$. Fix a maximal torus $\mathrm{T}$ of $\mathrm{K}$ and a chamber $\mathcal{C}$ in the dual of the Cartan subalgebra $t$. Let $\mathcal{C}^{\vee}$ be the dual chamber in $\mathfrak{t}$ and let $\mathcal{A}$ be the unique alcove in $\mathrm{C}^{\vee}$ such that the origin is in the closure of $\mathcal{A}$.

Centralizers. Let $\mathrm{K}_{\mathrm{g}}=\{\mathrm{h} \in \mathrm{K} \mid \mathrm{hg}=\mathrm{gh}\}$ denote the centralizer of $\mathrm{g} \in \mathrm{K}$.

A.1. Lemma. The centralizer $\mathrm{K}_{\mathrm{g}}$ is connected for all $\mathrm{g} \in \mathrm{K}$. Let $\sigma$ be a (relatively open) face of the alcove $\mathcal{A}$. Then $\mathrm{K}_{\exp \xi}=\mathrm{K}_{\exp \eta}$ for all $\xi, \eta \in \sigma$.

Proof. The first assertion is [Bor61] Corollaire 3.5]. (Cf. also [Bou82, Ch. 9, § 5.3, Corollaire 1].) Let $\xi \in \sigma$ and put $\mathrm{g}=\exp \xi$. To prove the second assertion we need only show that the Lie algebra $\mathfrak{k}_{g}=\operatorname{ker}(\operatorname{Ad}(g)-1)$ of $K_{g}$ is independent of the point $\xi \in \sigma$. Let $R$ denote the root system of the pair $(K, T)$ and $\mathfrak{g}=\mathfrak{k}^{\mathrm{C}}$ the complexification of $\mathfrak{k}$. Since $g$ is in the torus $T, K_{g}$ contains $T$, so the root-space decomposition $\mathfrak{g}=\mathfrak{t}^{\mathrm{C}} \oplus \bigoplus_{\alpha \in \mathrm{R}} \mathfrak{g}_{\alpha}$ restricts to the root-space decomposition

$$
\left(\mathfrak{k}_{\mathrm{g}}\right)^{\mathrm{C}}=\mathfrak{t}^{\mathrm{C}} \oplus \bigoplus_{\alpha \in \mathrm{R}_{\xi}} \mathfrak{g}_{\alpha}
$$

where $R_{\xi} \subseteq R$ is the root system of $\left(K_{g}, T\right)$. To determine $R_{\xi}$ choose a root vector $x_{\alpha} \in \mathfrak{g}_{\alpha}$ for each $\alpha \in R$. Then $x_{\alpha} \in\left(\mathfrak{k}_{g}\right)^{\mathrm{C}}$ if and only if $e^{\text {ad } \xi_{\alpha}} x_{\alpha}=\operatorname{Ad}(g) x_{\alpha}=x_{\alpha}$. Identifying as usual a root $\alpha: T \rightarrow \mathbf{U}(1)$ with its differential $\mathrm{T}_{1} \alpha: \mathfrak{t} \rightarrow \boldsymbol{i} \mathbf{R}$ we have $\left[\xi, x_{\alpha}\right]=\alpha(\xi) x_{\alpha}$, so

$$
R_{\xi}=\{\alpha \in R \mid \alpha(\xi) \in 2 \pi i Z\} .
$$

Now let

$$
R_{\sigma}=\{\alpha \in R \mid \alpha(\eta) \in 2 \pi i Z \text { for all } \eta \in \sigma\} .
$$

To finish the proof it suffices to show that $R_{\xi}=R_{\sigma}$. Clearly $R_{\sigma} \subseteq R_{\xi}$. Let $\alpha \in R_{\xi}$. Then $\alpha(\xi)=2 \pi$ in with $n \in \mathbf{Z}$, so $\xi$ is in the singular hyperplane

$$
\mathrm{H}_{\alpha, n}=\{\eta \in \mathfrak{t} \mid \alpha(\eta)=2 \pi \mathfrak{i n}\} .
$$

The intersection of $\mathrm{H}_{\alpha, n}$ with the closed alcove $\overline{\mathcal{A}}$ is the closure of a face $\tau$ of $\mathcal{A}$. Since $\xi \in \sigma, \sigma$ intersects the closure of $\tau$ and therefore is a face of $\tau$. Hence $\sigma \subseteq$ $H_{\alpha, n}$. This implies $\alpha(\eta)=2 \pi i n$ for all $\eta \in \sigma$, so $\alpha \in R_{\sigma}$.

This result is well-known to be false if $K$ is not simply connected. One need only think of a rotation by $\theta$ in $\mathbf{S O}(3)$. If $\theta$ is not $\pi$, the centralizer is $\mathbf{S O}(2)$; if it is $\pi$, the centralizer is $\mathbf{O}(2)$. 
If $\sigma$ is the face containing $\xi \in \overline{\mathcal{A}}$ we shall permit ourselves to call $\mathrm{K}_{\exp \xi}$ the centralizer of the face $\sigma$. We denote it by $K_{\sigma}$ and its Lie algebra by $\mathfrak{k}_{\sigma}$. Its root system $R_{\sigma}$ is given by (A.2), so its complexified Lie algebra is

$$
\left(\mathfrak{k}_{\sigma}\right)^{\mathrm{C}}=\mathfrak{t}^{\mathrm{C}} \oplus \bigoplus_{\alpha \in \mathrm{R}_{\sigma}} \mathfrak{g}_{\alpha} .
$$

The proof of Lemma A.1 also yields the following useful information. Here $Z(G)$ denotes the centre and $G^{0}$ the identity component of a Lie group $G$, and $\mathfrak{z}(\mathfrak{g})$ denotes the centre of a Lie algebra $\mathfrak{g}$. For simplicity we denote the face $\{0\}$ of $\mathcal{A}$ by 0 .

A.3. Lemma. Let $\sigma$ and $\tau$ be faces of the alcove $\mathcal{A}$.

(i) If $\sigma \leq \tau$, then $\mathrm{K}_{\tau} \subseteq \mathrm{K}_{\sigma}$.

(ii) If $\sigma \geq 0$ and $\sigma^{\prime}$ is the unique face of the chamber $\mathrm{e}^{\vee}$ containing $\sigma$, then $\mathrm{K}_{\sigma}=$ $\mathrm{K}_{\sigma^{\prime}}$, the centralizer of $\sigma^{\prime}$ for the adjoint action of $\mathrm{K}$ on $\mathfrak{k}$.

(iii) Let $\mathrm{H}_{\sigma}$ be the affine subspace of $\mathfrak{t}$ spanned by $\sigma$. Then the linear subspace of $\mathfrak{t}$ parallel to $\mathrm{H}_{\sigma}$ is $\mathfrak{z}\left(\mathfrak{k}_{\sigma}\right)$, and exp $\mathrm{H}_{\sigma}$ is contained in $\mathrm{Z}\left(\mathrm{K}_{\sigma}\right)$. If $\sigma \geq 0$, then $\mathrm{H}_{\sigma}=\mathfrak{z}\left(\mathfrak{k}_{\sigma}\right)$ and $\exp \mathrm{H}_{\sigma}=\mathrm{Z}\left(\mathrm{K}_{\sigma}\right)^{0}$.

(iv) There exists $\mathrm{g}_{\sigma} \in\left[\mathrm{K}_{\sigma}, \mathrm{K}_{\sigma}\right] \cap \mathrm{Z}\left(\mathrm{K}_{\sigma}\right)$, unique up to multiplication by an element of $\Gamma_{\sigma}=\left[\mathrm{K}_{\sigma}, \mathrm{K}_{\sigma}\right] \cap \mathrm{Z}\left(\mathrm{K}_{\sigma}\right)^{0}$, such that $\exp \mathrm{H}_{\sigma} \subseteq \mathrm{g}_{\sigma} \mathrm{Z}\left(\mathrm{K}_{\sigma}\right)^{0}$.

Proof. By A.2 $\sigma \leq \tau$ implies $R_{\tau} \subseteq R_{\sigma}$, and hence $K_{\tau} \subseteq K_{\sigma}$.

Assume $\sigma \geq 0$ and let $\alpha \in R_{\sigma}$. Then $\alpha(\xi)=0$ for any $\xi \in \sigma$. Letting $\alpha_{1}, \alpha_{2}, \ldots$, $\alpha_{r}$ be the simple roots with respect to the chamber $\mathcal{C}$ and writing $\alpha=\sum_{i=1}^{r} n_{i} \alpha_{i}$ with all $n_{i}$ having the same sign, we see that $\alpha_{i}(\xi)=0$ if $n_{i} \neq 0$. So $\alpha$ is a combination of the simple roots annihilating $\xi$. This implies $\mathrm{K}_{\sigma}=\mathrm{K}_{\xi}=\mathrm{K}_{\sigma^{\prime}}$.

For $\alpha \in R_{\sigma}$ denote by $\alpha(\sigma) \in\{0, \pm 2 \pi i\}$ the constant value of $\alpha$ on $\sigma$. Then $H_{\sigma}$ is the intersection of the affine hyperplanes $H_{\alpha, \sigma(\alpha)}$ over all $\alpha \in R_{\sigma}$, i.e.

$$
\mathrm{H}_{\sigma}=\left\{\xi \in \mathfrak{t} \mid \alpha(\xi)=\alpha(\sigma) \text { for all } \alpha \in R_{\sigma}\right\} .
$$

On the other hand $\mathfrak{z}\left(\mathfrak{k}_{\sigma}\right)=\bigcap\left\{\operatorname{ker} \alpha \mid \alpha \in R_{\sigma}\right\}$, so $H_{\sigma}$ is a translate $\xi+\mathfrak{z}\left(\mathfrak{k}_{\sigma}\right)$ for some $\xi \in H_{\sigma}$. In fact, we can take $\xi \in \sigma$ and therefore $\exp H_{\sigma} \subseteq Z\left(K_{\sigma}\right)$ since $\exp \xi \in Z\left(K_{\sigma}\right)$. If $\sigma \geq 0$ then $0 \in \mathrm{H}_{\sigma}$, so $\mathrm{H}_{\sigma}=\mathfrak{z}\left(\mathfrak{k}_{\sigma}\right)$ and therefore $\exp \mathrm{H}_{\sigma}=\mathrm{Z}\left(\mathrm{K}_{\sigma}\right)^{0}$.

Let $\xi$ be the closest point to the origin in the affine subspace $H_{\sigma}$ (relative to the distance function given by an invariant inner product on $\mathfrak{k})$ and put $g_{\sigma}=\exp \xi$. Then $g_{\sigma} \in Z\left(K_{\sigma}\right)$ by (iii). Furthermore, $\xi$ is perpendicular to the linear subspace parallel to $\mathrm{H}_{\sigma}$, i.e. $\xi \in \mathfrak{z}\left(\mathfrak{k}_{\sigma}\right)^{\perp}=\left[\mathfrak{k}_{\sigma}, \mathfrak{k}_{\sigma}\right]$. We conclude that $g_{\sigma} \in\left[K_{\sigma}, K_{\sigma}\right] \cap Z\left(K_{\sigma}\right)$. Since $H_{\sigma}=\xi+\mathfrak{z}\left(\mathfrak{k}_{\sigma}\right)$ we have $\exp H_{\sigma} \subseteq g_{\sigma} Z\left(K_{\sigma}\right)^{0}$. The uniqueness of $g_{\sigma}$ up to $\Gamma_{\sigma}$ is obvious.

It is now a simple matter to construct a base (set of simple roots) for each of the root systems $R_{\sigma}$. Let $B$ be the base and $R_{\min }$ the set of minimal roots of $R$ determined by the chamber $\mathcal{C}$. ( $R_{\min }$ contains one element in each irreducible component of R.) By a wall of an open polyhedron $P$ in a vector space $V$ (e.g. $P$ is a chamber or an alcove in $t$ ) we mean an affine hyperplane of $V$ spanned by a codimension-one face of $\mathrm{P}$. Recall that the walls of $\mathrm{C}^{\vee}$ are the hyperplanes $\mathrm{H}_{\alpha, 0}$ with $\alpha \in \mathrm{B}$, while the walls of $\mathcal{A}$ include the walls of $\mathrm{C}^{\vee}$ as well as the hyperplanes $\mathrm{H}_{\alpha,-1}$ with $\alpha \in \mathrm{R}_{\min }$. Let

$$
B_{\sigma}=\left(R_{\sigma} \cap R_{\min }\right) \cup\left\{\alpha \in R_{\sigma} \cap B \mid \alpha(\sigma)=0\right\},
$$


where $\alpha(\sigma)$ denotes the (constant) value of any $\alpha \in R_{\sigma}$ on $\sigma$. Note that $\sigma \geq 0 \Longleftrightarrow$ $\mathrm{B}_{\sigma} \subseteq \mathrm{B}$.

A.4. Lemma. Let $\sigma$ be a face of $\mathcal{A}$. Then $\mathrm{B}_{\sigma}$ is a base of $\mathrm{R}_{\sigma}$. Hence $\left|\mathrm{B}_{\sigma}\right|=\operatorname{rank}\left[\mathrm{K}_{\sigma}, \mathrm{K}_{\sigma}\right]$ and $\left|\mathrm{B}_{\sigma}\right|+\operatorname{dim} \sigma=\operatorname{rank} \mathrm{K}$.

Proof. Let $\mathcal{H}_{\sigma}$ be the collection of singular hyperplanes of $\mathfrak{t}$ which contain $\sigma$ and let $\mathcal{C}_{\sigma}^{\vee}$ be the connected component of $\mathfrak{t}-\bigcup \mathcal{H}_{\sigma}$ containing $\mathcal{A}$. Since $\exp \sigma \subseteq \mathrm{Z}\left(\mathrm{K}_{\sigma}\right)$, $\mathcal{C}_{\sigma}^{\vee}$ is (up to translation by any element of $\sigma$ ) a chamber of $\left(K_{\sigma}, T\right)$. The walls of the cone $\mathcal{C}_{\sigma}^{\vee}$ are the same as the walls of $\mathcal{A}$ which contain $\sigma$, which are exactly the hyperplanes $\mathrm{H}_{\alpha, \alpha(\sigma)}$ with $\alpha \in \mathrm{B}_{\sigma}$. Therefore the elements of $\mathrm{B}_{\sigma}$ are the simple roots of $\left(K_{\sigma}, T\right)$ determined by the chamber $\mathcal{C}_{\sigma}^{\vee}$. In particular $B_{\sigma}$ spans the Lie algebra of the maximal torus $\left[\mathrm{K}_{\sigma}, \mathrm{K}_{\sigma}\right] \cap \mathrm{T}$ of $\left[\mathrm{K}_{\sigma}, \mathrm{K}_{\sigma}\right]$ and hence $\left|\mathrm{B}_{\sigma}\right|=\operatorname{rank}\left[\mathrm{K}_{\sigma}, \mathrm{K}_{\sigma}\right]$. By Lemma A.3 iii), $\operatorname{dim} \sigma=\operatorname{dim} Z\left(K_{\sigma}\right)$, so $\left|B_{\sigma}\right|+\operatorname{dim} \sigma=\operatorname{rank} K_{\sigma}=\operatorname{rank} K$.

Lemma A.3 i) can be much improved for a face of dimension greater than one. It turns out that the centralizer of such a face is determined by the centralizers of its proper subfaces. Let us write $\sigma<\tau$ if $\sigma \leq \tau$ and $\sigma \neq \tau$.

A.5. Proposition. Let $\tau$ be a face of $\mathcal{A}$ of dimension at least two. Then

(i) $K_{\tau}=\bigcap_{\sigma<\tau} K_{\sigma}$;

(ii) $\left[K_{\tau}, K_{\tau}\right]=\bigcap_{\sigma<\tau}\left[K_{\sigma}, K_{\sigma}\right]$.

Proof. The inclusion $\mathrm{K}_{\tau} \subseteq \bigcap_{\sigma<\tau} \mathrm{K}_{\sigma}$ follows from Lemma A.3 i). Conversely, suppose $g \in K_{\sigma}$ for all $\sigma<\tau$. Then $\operatorname{Ad}(g) \exp \xi=\exp \xi$ for all $\xi$ in the (combinatorial) boundary $\partial \tau$ of $\tau$. Hence for each $\xi \in \partial \tau$ there exists $\gamma$ in the exponential lattice $\Gamma(\mathrm{T})=\exp _{\mathrm{T}}^{-1}(1)$ such that $\operatorname{Ad}(\mathrm{g}) \xi=\xi+\gamma$. The boundary of $\tau$ is connected because $\operatorname{dim} \tau \geq 2$. The map $\operatorname{Ad}(\mathrm{g})$ being continuous, we conclude that $\gamma$ is independent of $\xi \in \partial \tau$. Let $\eta \in \tau$ and write $\eta$ as a convex combination $\eta=\sum_{i} c_{i} \xi_{i}$ with $\xi_{i} \in \partial \tau$ and $\sum_{i} c_{i}=1$. Then

$$
\operatorname{Ad}(g) \eta=\sum_{i} c_{i} \operatorname{Ad}(g) \xi_{i}=\sum_{i} c_{i}\left(\xi_{i}+\gamma\right)=\eta+\gamma
$$

and therefore $\operatorname{Ad}(\mathrm{g}) \exp \eta=\exp \eta$, i.e. $g \in \mathrm{K}_{\tau}$. Thus $\bigcap_{\sigma<\tau} \mathrm{K}_{\sigma} \subseteq \mathrm{K}_{\tau}$.

Put $\mathrm{H}=\bigcap_{\sigma<\tau}\left[\mathrm{K}_{\sigma}, \mathrm{K}_{\sigma}\right]$. Then $\mathrm{H} \subseteq \bigcap_{\sigma<\tau} \mathrm{K}_{\sigma}=\mathrm{K}_{\tau}$ by (i). In addition Lemma A.3 i) implies $\left[K_{\tau}, K_{\tau}\right] \subseteq\left[K_{\sigma}, K_{\sigma}\right]$ for $\sigma \leq \tau$ and hence $\left[K_{\tau}, K_{\tau}\right] \subseteq H$. Now $\left[K_{\tau}, K_{\tau}\right] \subseteq$ $\mathrm{H}$ implies that $\mathrm{H}$ (as well as $\left[\mathrm{K}_{\tau}, \mathrm{K}_{\tau}\right]$ ) is a normal subgroup of $\mathrm{K}_{\tau}$ and therefore, $\mathrm{T}$ being a maximal torus of $\mathrm{K}_{\tau}$, the reverse inclusion $\mathrm{H} \subseteq\left[\mathrm{K}_{\tau}, \mathrm{K}_{\tau}\right]$ will follow from

$$
\mathrm{H} \cap \mathrm{T} \subseteq\left[\mathrm{K}_{\tau}, \mathrm{K}_{\tau}\right] .
$$

To prove this we may assume without loss of generality that $\mathrm{K}$ is almost simple, i.e. that $R$ is irreducible. Let $x \in H \cap T$. Since $\operatorname{dim} \tau \geq 2$, $\tau$ has at least two codimensionone faces $\rho$ and $\sigma$, so we can write $x=\exp \xi=\exp \eta$ where $\xi \in\left[\mathfrak{k}_{\rho}, \mathfrak{k}_{\rho}\right] \cap \mathfrak{t}$ and $\eta \in\left[\mathfrak{k}_{\sigma}, \mathfrak{k}_{\sigma}\right] \cap \mathfrak{t}$. Thus $\xi-\eta$ is in the exponential lattice $\Gamma(T)$, which is equal to the coroot lattice $Q\left(R^{\vee}\right)$ since $K$ is simply connected. The bases of the root systems $R_{\rho}$ and $R_{\sigma}$ are of the form $B_{\rho}=B_{\tau} \cup\{\alpha\}$ and $B_{\sigma}=B_{\tau} \cup\{\beta\}$ where $\alpha, \beta \in B \cup R_{\text {min }}$ are two distinct roots not contained in $B_{\tau}$. Hence $\xi=\xi_{0}+a \alpha^{\vee}$ and $\eta=\eta_{0}+b \beta^{\vee}$ with $\xi_{0}, \eta_{0} \in\left[\mathfrak{k}_{\tau}, \mathfrak{k}_{\tau}\right] \cap \mathfrak{t}$ and $a, b \in i \mathbf{R}$. Thus

$$
\xi_{0}-\eta_{0}+a \alpha^{\vee}-b \beta^{\vee}=\xi-\eta \in Q\left(R^{\vee}\right) .
$$


First suppose that $\tau \geq 0$. Then we can choose $\rho$ and $\sigma$ so that $\rho \geq 0$ and $\sigma \geq 0$. This implies that $\alpha, \beta \in B$ are simple and therefore $a, b \in 2 \pi i Z$ by A.7. We conclude that $x=\exp \xi_{0} \in\left[K_{\tau}, K_{\tau}\right]$.

Now suppose that $\tau \nsupseteq 0$. Then, since $R$ is irreducible, $B_{\tau}$ contains the unique minimal root $\alpha_{0}$ and so $\alpha$ and $\beta$ are simple. In fact, $\tau$ has at least one more codimension-one face $v$ distinct from $\rho$ and $\sigma$; we have $B_{v}=B_{\tau} \cup\{\gamma\}$ with $\gamma \in B$ distinct from $\alpha$ and $\beta$; and we can write $x=\exp \zeta$ with $\zeta=\zeta_{0}+c \gamma^{\vee}, \zeta_{0} \in\left[\mathfrak{k}_{\tau}, \mathfrak{k}_{\tau}\right] \cap \mathfrak{t}$ and $c \in i \boldsymbol{R}$. Expanding the coroot $-\alpha_{0}^{\vee}=\sum_{\delta \in B} m_{\delta} \delta^{\vee}$ in the dual base $B^{\vee}$ with positive integer coefficients $m_{\delta}$ we find using A.7 that the coefficient of $\alpha_{0}^{\vee}$ in $\xi_{0}-\eta_{0}$ is an integer multiple of $2 \pi i / m_{\gamma}$ and therefore $a \in 2 \pi i Z\left(1, m_{\alpha} / m_{\gamma}\right)$. Permuting $\alpha, \beta$ and $\gamma$ we get the integrality conditions

$$
\begin{gathered}
a / 2 \pi i \in \mathbf{Z}\left(1, m_{\alpha} / m_{\beta}\right) \cap \mathbf{Z}\left(1, m_{\alpha} / m_{\gamma}\right), \quad b / 2 \pi i \in \mathbf{Z}\left(1, m_{\beta} / m_{\gamma}\right) \cap \mathbf{Z}\left(1, m_{\beta} / m_{\alpha}\right), \\
c / 2 \pi i \in \mathbf{Z}\left(1, m_{\gamma} / m_{\alpha}\right) \cap \mathbf{Z}\left(1, m_{\gamma} / m_{\beta}\right) .
\end{gathered}
$$

By looking at the values of the coefficients $m_{\delta}$ for each irreducible root system (see table in Example 5.4) one can check that these conditions imply that for all triples of distinct simple roots $\alpha, \beta, \gamma$ at least one of the numbers $a, b, c$ is in $2 \pi i Z$ and hence that $x \in\left[\mathrm{K}_{\tau}, \mathrm{K}_{\tau}\right]$. This proves (A.6).

Slices. Using Lemma A.3 we can define a large "logarithmic" chart on K. Let O be the $W$-invariant open neighbourhood of the origin in $t$ consisting of all $\xi$ with $(2 \pi i)^{-1} \alpha(\xi)<1$ for all roots $\alpha$ and let $\mathrm{U}=\operatorname{Ad}(K) O=\operatorname{Ad}(K)\left(O \cap \overline{\mathcal{C}}^{\vee}\right)$.

\section{A.8. Lemma. $\quad$ (i) $\mathrm{O} \cap \mathrm{e}^{\vee}=\mathcal{A}$ and $\mathrm{O} \cap \overline{\mathcal{C}}^{\vee}=\bigcup\{\sigma \mid 0 \leq \sigma \leq \mathcal{A}\}$.}

(ii) $\exp \mathrm{U}$ is open and dense in $\mathrm{K}$ and exp: $\mathrm{U} \rightarrow \exp \mathrm{U}$ is a local diffeomorphism.

(iii) $\exp : \frac{1}{2} \mathrm{U} \rightarrow \mathrm{K}$ is a diffeomorphism onto its image.

Proof. Let $\mathrm{R}_{+}$denote the set of positive roots with respect to the chamber $\mathrm{C}$. By [Bou82. Ch. 6, $\S 2.3$, Proposition 5] a point $\xi \in \mathfrak{t}$ is in the alcove $\mathcal{A}$ if and only if $0<(2 \pi i)^{-1} \alpha(\xi)<1$ for all $\alpha \in R_{+}$. Let us abbreviate this system of inequalities for $\mathcal{A}$ to

$$
0<(2 \pi i)^{-1} \alpha<1 \quad \text { for } \alpha \in R_{+} .
$$

Likewise, the chamber $\mathcal{C}^{\vee}$ in $\mathfrak{t}$ is given by $(2 \pi \mathfrak{i})^{-1} \alpha>0$ for $\alpha \in R_{+}$. Thus $\xi \in$ $\mathrm{O} \cap \mathrm{C}^{\vee}$ iff $0<(2 \pi \mathrm{i})^{-1} \alpha(\xi)<1$ for all $\alpha \in \mathrm{R}_{+}$iff $\xi \in \mathcal{A}$. Hence $\mathrm{O} \cap \mathcal{C}^{\vee}=\mathcal{A}$. This implies $\mathrm{O} \cap \overline{\mathrm{e}}^{\vee} \subseteq \overline{\mathcal{A}}$; in fact

$$
\xi \in \mathrm{O} \cap \overline{\mathrm{C}}^{\vee} \Longleftrightarrow 0 \leq(2 \pi \mathrm{i})^{-1} \alpha(\xi)<1 \text { for all } \alpha \in \mathrm{R}_{+} .
$$

So if $\sigma \leq \mathcal{A}$ is the face containing $\xi \in \mathrm{O} \cap \overline{\mathrm{C}}^{\vee}$ then $\alpha(\sigma)=0$ for all $\alpha \in \mathrm{R}_{\sigma}$, and hence $\sigma \geq 0$. Conversely, if $\xi \in \overline{\mathcal{A}}$ is contained in a face $\sigma \geq 0$, then $(2 \pi i)^{-1} \alpha(\xi)<1$ for all $\alpha \in \mathrm{R}_{+}$, so $\xi \in \mathrm{O} \cap \overline{\mathrm{e}}^{\vee}$. Thus $\mathrm{O} \cap \overline{\mathrm{e}}^{\vee}=\bigcup\{\sigma \mid 0 \leq \sigma \leq \mathcal{A}\}$.

The formula $T_{\xi} \exp =T_{1} L(\exp \xi) \circ\left(1-e^{-a d \xi}\right) /$ ad $\xi$ shows that $T_{\xi}$ exp is bijective if and only if ad $\xi$ does not have an eigenvalue $2 \pi i n$ with $n \in \mathbf{Z}-\{0\}$. This implies that $T_{\xi} \exp$ is bijective for every point in $U$. Therefore exp $U$ is open and exp: $U \rightarrow$ $\exp U$ is a local diffeomorphism. Since exp $U$ contains the set of regular elements $\operatorname{Ad}(\mathrm{K}) \exp \mathcal{A}$, it is dense in $\mathrm{K}$.

It remains to show that exp: $\frac{1}{2} \mathrm{U} \rightarrow \mathrm{K}$ is injective. Suppose $\xi, \eta \in \frac{1}{2} \mathrm{U}$ satisfy $\exp \xi=\exp \eta$. Without loss of generality we may assume $\xi \in \frac{1}{2} \mathrm{O} \cap \overline{\mathrm{C}}^{\vee}$ and $\eta=\operatorname{Ad}(g) \xi^{\prime}$ with $g \in K$ and $\xi^{\prime} \in \frac{1}{2} \mathrm{O} \cap \overline{\mathcal{C}}^{\vee}$. Then $\exp \xi$, $\exp \xi^{\prime} \in \mathrm{T}$, so $\exp \xi=\operatorname{Ad}(g) \exp \xi^{\prime}$ implies that $\exp \xi=\operatorname{Ad}(n) \exp \xi^{\prime}$ for some $n \in N(T)$. Then 
$\mathrm{gn}^{-1}$ is in the centralizer of $\exp \xi^{\prime}$, which by (i) and LemmaA.3 ii) is equal to the centralizer of $\xi^{\prime}$. Therefore

$$
\eta=\operatorname{Ad}(g) \xi^{\prime}=w \xi^{\prime}
$$

where $w$ is the Weyl group element represented by $n$. From $\exp \xi=\operatorname{Ad}(n) \exp \xi^{\prime}$ we also get $\xi=w \xi^{\prime}+\gamma$ for some $\gamma \in \Gamma(T)$, where $\Gamma(T)=\exp _{\mathrm{T}}^{-1}(1)$ is the exponential lattice. Since $\xi, \xi^{\prime} \in \frac{1}{2} \mathrm{O} \cap \overline{\mathrm{C}}$ we have

$$
-1<\frac{\alpha\left(w \xi^{\prime}\right)}{\pi i}<1, \quad-1<\frac{\alpha(\xi)}{\pi i}=\frac{\alpha\left(w \xi^{\prime}\right)+\alpha(\gamma)}{\pi i}<1
$$

for all $\alpha \in R_{+}$. Since $\alpha(\gamma) \in 2 \pi i Z$ this implies $\alpha(\gamma)=0$ for all $\alpha$, so $\gamma=0$ and $\xi=w \xi^{\prime}$. Together with A.10 this shows $\xi=\eta$.

Let star $\sigma=\bigcup_{\tau \geq \sigma} \tau$ denote the open star of a face $\sigma \leq \mathcal{A}$ and put

$$
\mathfrak{S}_{\sigma}=\operatorname{Ad}\left(\mathrm{K}_{\sigma}\right) \exp (\operatorname{star} \sigma) \subseteq \mathrm{K}_{\sigma} .
$$

(Note that $\operatorname{star} \sigma=\mathrm{O} \cap \overline{\mathrm{e}}^{\vee}$ and $\mathfrak{S}_{\sigma}=\mathrm{U}$ for $\sigma=0$.) The next result (stated without proof in AMM98]) says that $\mathfrak{S}_{\sigma}$ is a slice for the conjugation action of $\mathrm{K}$ at all points in $\exp \sigma$.

A.11. Proposition. Let $\sigma$ be a face of $\mathcal{A}$. Then $\mathfrak{S}_{\sigma}$ is a submanifold of $\mathrm{K}$. Its orbit $\operatorname{Ad}(\mathrm{K}) \mathfrak{S}_{\sigma}$ under the conjugation action is open in $\mathrm{K}$. The conjugation map $\mathrm{K} \times \mathfrak{S}_{\sigma} \rightarrow \mathrm{K}$ descends to a diffeomorphism from the associated bundle $\mathrm{K} \times \mathrm{K}_{\sigma} \mathfrak{S}_{\sigma}$ onto $\operatorname{Ad}(\mathrm{K}) \mathfrak{S}_{\sigma}$. In particular, if $\mathrm{g} \in \mathfrak{S}_{\sigma}$ and $\mathrm{kgk}^{-1} \in \mathfrak{S}_{\sigma}$, then $\mathrm{k} \in \mathrm{K}_{\sigma}$.

Proof. The face $\sigma$ and its star can be described by inequalities similar to A.9. Let $R_{\sigma,+}=R_{\sigma} \cap R_{+}$be the set of positive roots in $R_{\sigma}$. Then $\sigma$ is given by

$$
\begin{cases}0<(2 \pi i)^{-1} \alpha<1 & \text { for } \alpha \in R_{+}-R_{\sigma,+}, \\ \alpha=\alpha(\sigma) & \text { for } \alpha \in R_{\sigma,+},\end{cases}
$$

while star $\sigma$ is given by

$$
\begin{cases}0<(2 \pi i)^{-1} \alpha<1 & \text { for } \alpha \in R_{+}-R_{\sigma,+}, \\ 0 \leq(2 \pi i)^{-1} \alpha<1 & \text { for } \alpha \in R_{\sigma,+}, \alpha(\sigma)=0, \\ 0<(2 \pi i)^{-1} \alpha \leq 1 & \text { for } \alpha \in R_{\sigma,+}, \alpha(\sigma)=2 \pi i .\end{cases}
$$

Let $\mathcal{A}_{\sigma} \subseteq \mathfrak{t}$ be the unique alcove for the subgroup $\mathrm{K}_{\sigma}$ containing $\mathcal{A}$. By (A.9, $\mathcal{A}_{\sigma}$ is given by the inequalities $0<(2 \pi i)^{-1} \alpha<1$ for $\alpha \in R_{\sigma,+}$. Hence its closure $\overline{\mathcal{A}}_{\sigma}$ is given by $0 \leq(2 \pi i)^{-1} \alpha \leq 1$ for $\alpha \in R_{\sigma,+}$. Comparing with A.13 we see that star $\sigma$ is open in $\overline{\mathcal{A}}_{\sigma}$. Hence the image of star $\sigma$ in the quotient $\overline{\mathcal{A}}_{\sigma} / \pi_{1}\left(\mathrm{~K}_{\sigma}\right)$ is open. By [Bou82. Ch. 9, § 5.2, Corollaire 1], the exponential map $\overline{\mathcal{A}}_{\sigma} \rightarrow$ T induces a homeomorphism from $\overline{\mathcal{A}}_{\sigma} / \pi_{1}\left(\mathrm{~K}_{\sigma}\right)$ to $\mathrm{K}_{\sigma} / \operatorname{Ad}\left(\mathrm{K}_{\sigma}\right)$. We conclude that $\mathfrak{S}_{\sigma}$ is an open subset of $\mathrm{K}_{\sigma}$, and therefore an embedded submanifold of $\mathrm{K}$. Let $\mu: \mathrm{K} \times \mathfrak{S}_{\sigma} \rightarrow \mathrm{K}$ be the conjugation map and let $g \in \mathfrak{S}_{\sigma}$. A tangent vector in $\mathrm{T}_{(1, g)}\left(\mathrm{K} \times \mathfrak{S}_{\sigma}\right)=\mathfrak{k} \oplus \mathrm{T}_{\mathrm{g}} \mathrm{K}_{\sigma}$ is of the form $\left(\xi, R(g)_{*} \eta\right)$ with $\xi \in \mathfrak{k}$ and $\eta \in \mathfrak{k}_{\sigma}$. A computation yields

$$
\mathrm{T}_{(1, \mathrm{~g})} \mu\left(\xi, \mathrm{R}(\mathrm{g})_{*} \eta\right)=\mathrm{R}(\mathrm{g})_{*} \xi-\mathrm{L}(\mathrm{g})_{*} \xi+\mathrm{R}(\mathrm{g})_{*} \eta .
$$

Hence the kernel of $T_{(1, g)} \mu$ consists of all $(\xi, R(g) * \eta)$ with

$$
\eta=\operatorname{Ad}(g) \xi-\xi \text {. }
$$


Since $g \in \mathfrak{S}_{\sigma}$, we can write $g=\exp (\operatorname{Ad}(k) \zeta)$ with $k \in K_{\sigma}$ and $\zeta \in \operatorname{star} \sigma$. Then (A.14) is equivalent to $\eta^{\prime}=e^{\operatorname{ad}} \zeta \xi^{\prime}-\xi^{\prime}$, where $\eta^{\prime}=\operatorname{Ad}(k)^{-1} \eta$ and $\xi^{\prime}=\operatorname{Ad}(k)^{-1} \xi$. Decomposing $\xi^{\prime}=h+\sum_{\alpha \in R} c_{\alpha} x_{\alpha}$ with $h \in \mathfrak{t}$ we find

$$
\eta^{\prime}=e^{\operatorname{ad} \zeta} \xi^{\prime}-\xi^{\prime}=\sum_{\alpha} c_{\alpha}\left(e^{\alpha(\zeta)}-1\right) x_{\alpha} .
$$

Since $\zeta \in$ star $\sigma$, we have $0<(2 \pi i)^{-1} \alpha(\zeta)<1$ for all $\alpha \in R_{+}-R_{\sigma,+}$ by A.13. Also $\eta^{\prime} \in \mathfrak{k}_{\sigma}$, so by A.15 we have $c_{\alpha}=0$ for all $\alpha \in R-R_{\sigma}$. This means $\xi^{\prime} \in \mathfrak{k}_{\sigma}$, and therefore $\xi=\operatorname{Ad}(k) \xi^{\prime} \in \mathfrak{k}_{\sigma}$. Putting this together with

$$
\operatorname{ker} \mathrm{T}_{(1, \mathrm{~g})} \mu=\left\{\left(\xi, \mathrm{L}(\mathrm{g})_{*} \xi-\mathrm{R}(\mathrm{g})_{*} \xi\right) \mid \xi \in \mathfrak{k}_{\sigma}\right\} \text {, }
$$

which is exactly the kernel of the $\mathrm{K}_{\sigma}$-quotient map $\mathrm{K} \times \mathfrak{S}_{\sigma} \rightarrow \mathrm{K} \times \mathrm{K}_{\sigma} \mathfrak{S}_{\sigma}$. This shows that the map $\bar{\mu}: \mathrm{K} \times{ }^{K_{\sigma}} \mathfrak{S}_{\sigma} \rightarrow \mathrm{K}$ induced by $\mu$ is immersive at all points of the form $(1, g)$. Being $K$-equivariant $\bar{\mu}$ is an immersion at every point. Moreover, $\operatorname{dim} K \times{ }^{K_{\sigma}} \mathfrak{S}_{\sigma}=\operatorname{dim} K+\operatorname{dim} K_{\sigma}-\operatorname{dim} K_{\sigma}=\operatorname{dim} K$, so $\operatorname{Ad}(K) \mathfrak{S}_{\sigma}$ is open in $K$ and $\bar{\mu}$ is a local diffeomorphism. It remains to show that $\bar{\mu}$ is injective, which is equivalent to the last statement of the proposition. So let $k \in K, g \in \mathfrak{S}_{\sigma}$ and suppose $\mathrm{kgk}^{-1} \in \mathfrak{S}_{\sigma}$. Without loss of generality we may assume $\mathrm{g}=\exp \xi$ with $\xi \in \operatorname{star} \sigma$. We can write $k(\exp \xi) k^{-1}=h(\exp \eta) h^{-1}$ for certain $h \in K_{\sigma}$ and $\eta \in \operatorname{star} \sigma$. Both $\xi$ and $\eta$ are in $\overline{\mathcal{A}}$, so from the identification $\overline{\mathcal{A}} \cong \mathrm{K} / \operatorname{Ad}(\mathrm{K})$ we obtain $\eta=\xi$, whence $h^{-1} k \in K_{\exp \xi}$. Since $K_{\exp \xi} \subseteq K_{\sigma}$ by Lemma A.3 iil and $h \in K_{\sigma}$, we conclude $k \in K_{\sigma}$.

The action of the centre. The translation action of the centre $\mathrm{Z}(\mathrm{K})$ on $\mathrm{K}$ commutes with the adjoint action of $K$ and so descends to an action on the space of conjugacy classes. Via the identification $\mathrm{K} / \mathrm{Ad} \mathrm{K} \cong \exp \overline{\mathcal{A}}$ we can think of this action as taking place on $\exp \overline{\mathcal{A}}$, namely by shifting $\exp \overline{\mathcal{A}}$ by a central element and reflecting the resulting subset of T back into exp $\overline{\mathcal{A}}$ by a Weyl group element.

A.16. Lemma. For each $\mathrm{c} \in \mathrm{Z}(\mathrm{K})$ there exists a unique $w$ in the Weyl group $\mathrm{W}$ such that $w(\mathrm{~L}(\mathrm{c}) \exp \mathcal{A})=\exp \mathcal{A}$. The map $\zeta: \mathrm{Z}(\mathrm{K}) \rightarrow W$ sending $\mathrm{c}$ to $w$ is an injective homomorphism.

Proof. By [Bou82, Ch. 9, § 5.4, Proposition 5] the inverse image of the centre $Z(K)$ under $\exp _{T}: t \rightarrow T$ is the coweight lattice $P\left(R^{\vee}\right) \subseteq t$, the lattice dual to the root lattice in $t^{*}$ (whether or not $\mathrm{K}$ is simply connected). In other words

$$
Z(K)=\{\exp \xi \mid \xi \in \mathfrak{t} \text { and } \alpha(\xi) \in 2 \pi i Z \text { for all } \alpha \in R\} .
$$

Accordingly, let $c$ be central and write $c=\exp \xi$ with $\xi \in P\left(R^{\vee}\right)$. If $H_{\alpha, n}=\{\eta \in \mathfrak{t} \mid$ $\alpha(\eta)=2 \pi i n\}$ is a singular hyperplane of $t$, then $\xi+H_{\alpha, n}=H_{\alpha, n+\alpha(\xi)}$, so $P\left(R^{\vee}\right)$ preserves the collection of singular hyperplanes. Therefore $P\left(R^{\vee}\right)$ maps alcoves to alcoves, so there is an element $\tilde{w}$ of the affine Weyl group $W_{\text {affine }}$ mapping $\xi+\mathcal{A}$ to $\mathcal{A}$. Since $K$ is simply connected we have $W_{\text {affine }}=W \ltimes \Gamma(T)$. Hence we can write $\tilde{w}=w t_{\gamma}$ with $w \in W$ and $\gamma \in \Gamma(T)$, where $t_{\gamma}$ denotes the translation by $\gamma$. From $\tilde{\mathcal{w}}(\xi+\mathcal{A})=\mathcal{A}$ we obtain

$$
w(\mathrm{~L}(\mathrm{c}) \exp \mathcal{A})=w(\exp (\xi+\gamma+\mathcal{A}))=\exp (\tilde{w}(\gamma+\mathcal{A}))=\exp \mathcal{A}
$$

Since $\exp \mathcal{A}$ and $\mathrm{L}(\mathrm{c}) \exp \mathcal{A}$ are connected components of the set regular points of $T$ and $W$ acts simply transitively on those components, $w$ is unique and we can define $\zeta: Z(K) \rightarrow W$ by $\zeta(c)=w$. The left $Z(K)$-action on $T$ commutes with the 
$W$-action, so from $w(\mathrm{~L}(\mathrm{c}) \exp \mathcal{A})=\exp \mathcal{A}$ we get $w(\exp \mathcal{A})=\mathrm{L}(\mathrm{c})^{-1} \exp \mathcal{A}$. Hence if $c^{\prime}$ is another central element,

$$
\begin{array}{r}
\zeta\left(\mathrm{cc}^{\prime}\right)(\exp \mathcal{A})=\mathrm{L}\left(\mathrm{cc}^{\prime}\right)^{-1} \exp \mathcal{A}=\mathrm{L}\left(\mathrm{c}^{\prime}\right)^{-1} \mathrm{~L}(\mathrm{c})^{-1} \exp \mathcal{A}=\mathrm{L}\left(\mathrm{c}^{\prime}\right)^{-1} \zeta(\mathrm{c})(\exp \mathcal{A}) \\
=\zeta(\mathrm{c})\left(\mathrm{L}\left(\mathrm{c}^{\prime}\right)^{-1} \exp \mathcal{A}\right)=\zeta(\mathrm{c}) \zeta\left(\mathrm{c}^{\prime}\right)(\exp \mathcal{A}),
\end{array}
$$

which shows that $\zeta$ is a homomorphism. If $w=\zeta(c)=1$ then $\tilde{w}=w t_{\gamma}=t_{\gamma}$ is a translation and hence $\gamma+\xi+\mathcal{A}=\mathcal{A}$. This implies $\xi=-\gamma$, so $\xi \in \Gamma(\mathrm{T})$ and $\mathrm{c}=\exp \xi=1$. Therefore $\zeta$ is injective.

A.18. Example. Let $K=\mathbf{S U}(l+1)$ and let $T \cong \mathbf{U}(1)^{\mathrm{l}}$ be the standard maximal torus. Identify the Cartan subalgebra $t$ with the subspace $V=\left\{x \mid x_{1}+x_{2}+\cdots+x_{l+1}=0\right\}$ of $\mathbf{R}^{l+1}$ via the isomorphism $x \mapsto 2 \pi i \operatorname{diag}\left(x_{1}, x_{2}, \ldots, x_{l+1}\right)$. The simple roots are then $(2 \pi i)^{-1} \alpha_{j}(x)=x_{j}-x_{j+1}$ for $1 \leq j<l+1$, the minimal root is $(2 \pi i)^{-1} \alpha_{0}(x)=$ $-x_{1}+x_{l+1}$, so the alcove is the open simplex

$$
\mathcal{A}=\left\{x \in \mathrm{V} \mid \mathrm{x}_{\mathrm{l}+1}+1>\mathrm{x}_{1}>\mathrm{x}_{2}>\cdots>\mathrm{x}_{\mathrm{l}+1}\right\} .
$$

Let $\varepsilon_{1}, \varepsilon_{2}, \ldots, \varepsilon_{l+1}$ be the standard basis of $\mathbf{R}^{l+1}$. Then the vertices of $\mathcal{A}$ are

$$
\sigma_{j}=\sum_{k=1}^{j} \varepsilon_{k}-\frac{j}{l+1} \sum_{k=1}^{l+1} \varepsilon_{k} \quad \text { for } j=0,1, \ldots, l .
$$

(Note that under the isomorphism $\mathbf{R}^{l+1} \rightarrow\left(\mathbf{R}^{l+1}\right)^{*}$ given by the standard inner product the vertex $\sigma_{j}$ is mapped to the fundamental weight $\varpi_{j}$.) The centre of $K$ is the cyclic group generated by $c=\exp \sigma_{1}=e^{-2 \pi i /(l+1)} \mathrm{I}$; we have $c^{j}=\exp \sigma_{j}$. The barycentre of $\mathcal{A}$ is

$$
\beta=\frac{1}{l+1} \sum_{j=0}^{l} \sigma_{j}=\frac{1}{2 l+2}(l, l-2, \ldots, 2-l,-l) .
$$

It is straightforward to check that $w\left(\gamma+\sigma_{1}+\beta\right)=\beta$, where $\gamma=-\varepsilon_{1}+\varepsilon_{l+1} \in \Gamma(T)$ and $w$ is the cyclic permutation $w(x)=\left(x_{l+1}, x_{1}, x_{2}, \ldots, x_{l}\right)$. Thus $\zeta(c)=w$.

\section{APPENDIX B. THE SPINNING 2n-SPHERE}

Here we describe a quasi-Hamiltonian $\mathbf{U}(n)$-structure on $S^{2 n}$, which generalizes the $\mathbf{U}(2)$-structure on $\mathrm{S}^{4}$ of [AMW02, Appendix A] and [HJ00, Proposition 2.29]. We discovered this structure in the course of our work on the imploded cross-section of DU(n). Because the final result is easy to explain without employing the machinery of implosion we present it in this appendix. Its relationship to implosion is clarified in Example 4.23

We will obtain $S^{2 n}$ by gluing together two copies of a quasi-Hamiltonian $2 n-$ disc. This is similar to the construction of $S^{4}$ in AMW02, but the generalization from $S^{4}$ to $S^{2 n}$ is not entirely straightforward. To begin with, the gluing formula in [loc. cit.] does not generalize to higher dimensions and must be adapted. Moreover, it turns out that there is a second generalization of the $\mathbf{U}(2)$-structure on $\mathrm{S}^{4}$, namely to a $\mathbf{U}(\mathrm{n}, \mathbf{H}) \times \mathbf{U}(1)$-structure on the quaternionic projective space $\mathbf{H P}^{\mathrm{n}}$. This will be the subject of a future publication.

Let $T$ be the standard maximal torus of $\mathbf{U}(n)$. As invariant inner product on $\mathfrak{u}(n)$ we take $(\xi, \eta)=-\left(4 \pi^{2}\right)^{-1} \operatorname{tr}(\xi \eta)$. Consider $C^{\mathfrak{n}}$ with its standard Hermitian 
structure $z^{*} w=\sum_{j} \bar{z}_{j} w_{j}$ and its standard $\mathbf{U}(\mathrm{n})$-action. The corresponding symplectic form $\omega_{0}$ and moment map $\Phi_{0}$ are given by

$$
\omega_{0}=\operatorname{Im}\langle\cdot, \cdot\rangle=\frac{i}{2} \sum_{j} \mathrm{~d} z_{j} \mathrm{~d} \bar{z}_{j}, \quad \Phi_{0}(z)=-2 \pi^{2} i z z^{*},
$$

where we have used the inner product on $\mathfrak{u}(n)$ to write $\Phi_{0}$ as a map into $\mathfrak{u}(n)$. To produce a quasi-Hamiltonian structure we exponentiate: $\Phi=\exp \circ \Phi_{0}$ and $\omega=\omega_{0}+\Phi_{0}^{*} \varpi$. We determine where $\omega$ is minimally degenerate and write a more explicit formula for it as follows.

B.1. Lemma. Let $\mathrm{D}$ be the disc $\left\{z \in \mathrm{C}^{\mathfrak{n}} \mid\|z\|^{2}<1 / \pi\right\}$.

(i) $T_{\xi} \exp$ is surjective for all $\xi \in \Phi_{0}(\mathrm{D})$.

(ii) $(\mathrm{D}, \omega, \Phi)$ is a quasi-Hamiltonian $\mathbf{U}(\mathrm{n})$-manifold.

(iii) Let $\lambda$ be the $\mathbf{U}(\mathrm{n})$-invariant two-form on $\mathbf{C}^{\mathfrak{n}}-\{0\}$ given by

$$
\begin{aligned}
& \lambda=\mathrm{d} \log \|z\|^{2} \wedge \frac{1}{2} \operatorname{Im}\left(z^{*} \mathrm{~d} z\right)=\frac{i}{2\|z\|^{2}} \sum_{1 \leq i \leq k \leq n} \bar{z}_{j} z_{k} \mathrm{~d} z_{j} \mathrm{~d} \bar{z}_{k} . \\
& \text { Then } \omega=\lambda+\frac{\sin \left(2 \pi^{2}\|z\|^{2}\right)}{2 \pi^{2}\|z\|^{2}}\left(\omega_{0}-\lambda\right) .
\end{aligned}
$$

Note that $\omega$ is smooth even though $\lambda$ has a pole at the origin. In fact, the value of $\omega$ at the origin is equal to the standard symplectic form $\omega_{0}$.

Proof. The Hermitian matrix $z z^{*}$ has spectrum $\left(\|z\|^{2}, 0, \ldots, 0\right)$. Hence the matrix $\Phi_{0}(z)$ is conjugate to the diagonal matrix $\xi=-2 \pi^{2} i \operatorname{diag}\left(\|z\|^{2}, 0, \ldots, 0\right) \in \mathfrak{t}$. Thus if $\alpha$ is a root we have either $\alpha(\xi)= \pm 2 \pi^{2} i\|z\|^{2}$ or $\alpha(\xi)=0$. According to Lemma A.8 iii) $T_{\xi} \exp$ is surjective if $(2 \pi i)^{-1} \alpha(\xi)<1$ for all $\alpha$, i.e. if $z \in D$. This proves (ii). It follows that $\omega$ is minimally degenerate on $D$, which proves (iii. Because of $\mathbf{U}(\mathrm{n})$-invariance, for the proof of (iii) it suffices to consider vectors $z$ of the form $\left(z_{1}, 0, \ldots, 0\right)$. At such a point $z$ we have

$$
\lambda=\frac{i}{2} d z_{1} d \bar{z}_{1}
$$

and so

$$
\lambda+\frac{\sin \left(2 \pi^{2}\|z\|^{2}\right)}{2 \pi^{2}\|z\|^{2}}\left(\omega_{0}-\lambda\right)=\frac{i}{2} d z_{1} d \bar{z}_{1}+\frac{i \sin \left(2 \pi^{2}\|z\|^{2}\right)}{4 \pi^{2}\|z\|^{2}} \sum_{r=2}^{n} d z_{r} d \bar{z}_{r} .
$$

To compute $\omega$ we first calculate $\varpi \in \Omega^{2}(\mathfrak{u}(n))$, or rather its complex bilinear extension to an element of $\Omega_{\mathbf{C}}^{2}(\mathfrak{g l}(n, \mathbf{C}))$. Let $\lambda \in \mathfrak{t}$. Let $\left\{\varepsilon_{\mathfrak{j} k}\right\}_{1 \leq j, k \leq n}$ be the standard basis of $\mathfrak{g l}(n, C)$, with dual basis $\left\{\mathrm{d} \xi_{j k}\right\}_{1 \leq j, k \leq n}$ in $\left.\mathfrak{g l}(n, C)\right)^{*}$, and for $\mathfrak{j} \neq k$ let $\alpha_{j k}$ be the root $d \xi_{j j}-d \xi_{k k}$. It follows from (2.6) that $\varpi_{\lambda}\left(\varepsilon_{j j}, \varepsilon_{k k}\right)=0$ for all $j$ and $k$. Likewise for $j \neq k$ and $l \neq m$ we have

$$
\varpi_{\lambda}\left(\varepsilon_{j k}, \varepsilon_{l m}\right)=\left(f\left(\alpha_{j k}(\lambda)\right) \varepsilon_{j k}, \varepsilon_{l m}\right)=-\frac{f\left(\alpha_{j k}(\lambda)\right)}{4 \pi^{2}} \delta_{k l} \delta_{j m},
$$

where $f(x)=(x-\sinh x) / x^{2}$. Using

$$
f\left(\alpha_{j k}(\lambda)\right) \delta_{k l} \delta_{j m}=\sum_{r<s} f\left(\alpha_{r s}(\lambda)\right) d \xi_{r s} d \xi_{s r}\left(\varepsilon_{j k}, \varepsilon_{l m}\right)
$$


we conclude that for all $\lambda \in \mathfrak{t}$

$$
\varpi_{\lambda}=-\frac{1}{4 \pi^{2}} \sum_{r<s} f\left(\alpha_{r s}(\lambda)\right) d \xi_{r s} d \xi_{s r} .
$$

The pullback $\left(\Phi_{0}^{*} \varpi\right)_{z}$ is found by substituting $\lambda=\Phi_{0}(z)$ and $\xi_{r s}=-2 \pi^{2} i z_{r} \bar{z}_{s}$ into $\varpi$. This yields $\alpha_{r s}(\lambda)=-2 \pi^{2} i\left(\left|z_{r}\right|^{2}-\left|z_{s}\right|^{2}\right)$ and

$$
\mathrm{d} \xi_{\mathrm{rs}} \mathrm{d} \xi_{\mathrm{sr}}=-4 \pi^{4}\left(\left|z_{\mathrm{s}}\right|^{2} \mathrm{~d} z_{\mathrm{r}} \mathrm{d} \bar{z}_{\mathrm{r}}-\left|z_{\mathrm{r}}\right|^{2} \mathrm{~d} z_{\mathrm{s}} \mathrm{d} \bar{z}_{\mathrm{s}}+2 i \operatorname{Im}\left(\bar{z}_{\mathrm{r}} \bar{z}_{\mathrm{s}} \mathrm{d} z_{\mathrm{r}} \mathrm{d} z_{\mathrm{s}}\right)\right),
$$

whence

$$
\begin{aligned}
\left(\Phi_{0}^{*} \varpi\right)_{z}=\pi^{2} \sum_{1 \leq \mathrm{r}<s \leq n} f\left(-2 \pi^{2} i\left(\left|z_{\mathrm{r}}\right|^{2}-\left|z_{\mathrm{s}}\right|^{2}\right)\right)\left(\left|z_{\mathrm{s}}\right|^{2} \mathrm{~d} z_{\mathrm{r}} \mathrm{d} \bar{z}_{\mathrm{r}}\right. & \\
& \left.-\left|z_{\mathrm{r}}\right|^{2} \mathrm{~d} z_{\mathrm{s}} \mathrm{d} \bar{z}_{\mathrm{s}}+2 \mathrm{i} \operatorname{Im}\left(\bar{z}_{\mathrm{r}} \bar{z}_{\mathrm{s}} \mathrm{d} z_{\mathrm{r}} \mathrm{d} z_{\mathrm{s}}\right)\right) .
\end{aligned}
$$

Since $z_{2}=\cdots=z_{\mathfrak{n}}=0$ this simplifies to

$$
\left(\Phi_{0}^{*} \varpi\right)_{z}=-\pi^{2}\|z\|^{2} f\left(-2 \pi^{2} i\|z\|^{2}\right) \sum_{s=2}^{n} d z_{s} d \bar{z}_{s} .
$$

From $f(x)=(x-\sinh x) / x^{2}$ we get

$$
\left(\Phi_{0}^{*} \varpi\right)_{z}=\left(-\frac{i}{2}+\frac{i \sin \left(2 \pi^{2}\|z\|^{2}\right)}{4 \pi^{2}\|z\|^{2}}\right) \sum_{s=2}^{n} \mathrm{~d} z_{s} \mathrm{~d} \bar{z}_{s}
$$

and so

$$
\omega_{z}=\left(\omega_{0}\right)_{z}+\left(\Phi_{0}^{*} \varpi\right)_{z}=\frac{i}{2} d z_{1} d \bar{z}_{1}+\frac{i \sin \left(2 \pi^{2}\|z\|^{2}\right)}{4 \pi^{2}\|z\|^{2}} \sum_{r=2}^{n} d z_{r} d \bar{z}_{r} .
$$

\section{Comparing with (B.3) we obtain (iii).}

Now define a $\mathbf{U}(\mathfrak{n})$-equivariant diffeomorphism from the punctured disc $\mathrm{D}-\{0\}$ to itself by

$$
\phi(z)=-s(z) z, \quad \text { where } \quad s(z)=\frac{\sqrt{\pi^{-1}-\|z\|^{2}}}{\|z\|} .
$$

B.6. Lemma. $\phi^{*} \omega=-\omega$ and $\phi^{*} \Phi=i \circ \Phi$.

Proof. Let $\mathrm{p}(z)$ be the anti-Hermitian matrix $2 \pi i z z^{*} /\|z\|^{2}$. Then

$$
\Phi_{0}(\phi(z))=-2 \pi^{2} i s(z)^{2} z z^{*}=-p(z)-\Phi_{0}(z) .
$$

Note that $p(z)$ is $2 \pi i$ times the orthogonal projection onto the line spanned by $z$. Therefore $\exp p(z)=1$, whence $\Phi(\phi(z))=\exp \left(\Phi_{0}(\phi(z))\right)=\exp \left(-\Phi_{0}(z)\right)=$ $\Phi(z)^{-1}$. From (B.7) we also see

$$
\phi^{*} \omega=\phi^{*}\left(\omega_{0}+\Phi_{0}^{*} \varpi\right)=\phi^{*} \omega_{0}-p^{*} \varpi-\Phi_{0}^{*} \varpi .
$$

A straightforward computation yields $\phi^{*} \omega_{0}=s(z)^{2} \omega_{0}-\pi^{-1}\|z\|^{-2} \lambda$, and so

$$
\begin{aligned}
\phi^{*} \omega & =s(z)^{2} \omega_{0}-\frac{1}{\pi\|z\|^{2}} \lambda-p^{*} \varpi-\Phi_{0}^{*} \varpi \\
& =-\left(\omega_{0}+\Phi_{0}^{*} \varpi\right)+\frac{1}{\pi\|z\|^{2}}\left(\omega_{0}-\lambda\right)-p^{*} \varpi \\
& =-\omega+\frac{1}{\pi\|z\|^{2}}\left(\omega_{0}-\lambda\right)-p^{*} \varpi .
\end{aligned}
$$


It remains to show that

$$
p^{*} \varpi=\frac{1}{\pi\|z\|^{2}}\left(\omega_{0}-\lambda\right) .
$$

Because both sides are $\mathbf{U}(\mathfrak{n})$-invariant forms, it suffices to check this identity at points $z \in \mathbf{C}^{\mathfrak{n}}$ of the form $\left(z_{1}, 0, \ldots, 0\right)$. From (B.2) we see

$$
\frac{1}{\pi\|z\|^{2}}\left(\omega_{0}-\lambda\right)=\frac{i}{2 \pi\|z\|^{2}} \sum_{r=2}^{n} \mathrm{~d} z_{\mathrm{r}} \mathrm{d} \bar{z}_{\mathrm{r}}
$$

To find $\left(p^{*} \varpi\right)_{z}$ we substitute $\lambda=\Phi_{0}(z)$ and $\xi_{r s}=2 \pi i z_{r} \bar{z}_{s} /\|z\|^{2}$ into (B.4), which yields

$$
\left(p^{*} \varpi\right)_{z}=-\sum_{r=2}^{n} f(2 \pi i)\|z\|^{-2} d z_{r} d \bar{z}_{r}=\frac{i}{2 \pi\|z\|^{2}} \sum_{r=2}^{n} d z_{r} d \bar{z}_{r} .
$$

Together with (B.9) this gives (B.8).

This lemma tells us that the quasi-Hamiltonian disc $(D, \omega, \Phi)$ and its opposite $(D,-\omega, i \circ \Phi)$ can be glued together smoothly via the map $\phi$, resulting in a quasiHamiltonian structure on the $2 \mathrm{n}$-sphere. Because the gluing map $\phi$ sends $\mathrm{D}$ to itself, it can also be viewed as a diffeomorphism from $S^{2 n}$ to itself, which reverses the quasi-Hamiltonian structure. Under a suitable identification of $\mathrm{D} \amalg_{\phi} \mathrm{D}$ with $\mathrm{S}^{2 \mathrm{n}}$ this map is nothing but the antipodal map. To summarize,

B.10. Theorem. The $2 \mathrm{n}$-dimensional sphere with its standard $\mathbf{U}(\mathrm{n})$-action is a quasiHamiltonian $\mathbf{U}(\mathrm{n})$-manifold. The antipodal map is an anti-automorphism of the quasiHamiltonian structure.

Define the maps $\Phi_{1}: \mathrm{D} \rightarrow \mathbf{S U}(\mathrm{n})$ and $\Phi_{2}: \mathrm{D} \rightarrow \mathbf{Z U}(\mathrm{n})$ by

$$
\Phi_{1}(z)=\exp 2 \pi^{2} i\left(\frac{\|z\|^{2}}{n} \mathrm{I}-z z^{*}\right), \quad \Phi_{2}(z)=e^{-2 \pi^{2} i\|z\|^{2} / n} \mathrm{I}
$$

and observe that $\Phi=\Phi_{1} \Phi_{2}$. Hence, by Lemma $2.3 \mathrm{iv}, S^{2 n}$ is a quasi-Hamiltonian $\mathbf{S U}(n) \times \mathbf{Z U}(n)$-manifold with moment map $\Phi_{1} \times \Phi_{2}$. By Theorem 2.7 the reduction of $S^{2 n}$ with respect to the central circle $Z \mathbf{U}(n)$ is a quasi-Hamiltonian SU(n)-manifold. Reduction at the poles $z=0$ and $\|z\|^{2}=\pi^{-1}$ gives a quotient consisting of a single point, while reduction at an intermediate level $\|z\|^{2}=a$ gives a projective space $\mathbf{C P}^{n-1}$. Note that the form $\lambda$ of Lemma B.1 iiii vanishes on the $2 n-1$-sphere $\|z\|^{2}=a$. Therefore the induced two-form on the quotient is

$$
\frac{\sin \left(2 \pi^{2} a\right)}{2 \pi^{2} a} \sigma
$$

where $\sigma$ is a multiple of the Fubini-Study symplectic form. Thus we have found a family of quasi-Hamiltonian $\mathbf{S U}(n)$-structures on $\mathbf{C P}^{\mathrm{n}-1}$ for which the two-forms happen to be closed and non-degenerate (except for $a=1 / 2 \pi$, which corresponds to the equator of $S^{2 n}$, where the induced form is zero). It is easy to see (e.g. by using Addendum 3.18 that these quasi-Hamiltonian $\mathbf{S U}(n)$-structures are the same as those one obtains by considering $\mathbf{C P}^{\mathrm{n}-1}$ as a conjugacy class of $\mathbf{S U}(\mathrm{n})$. 


\section{NOTATION INDEX}

\author{
$<$, strict inclusion of faces, 30 \\ $\leq$, inclusion of faces, 6,8 \\ $\circledast$, fusion product, 4 \\ $\sim$, equivalence relation defining $M_{\text {impl }}, 7$ \\ $\mathcal{A}$, open alcove in $\mathrm{t}, 7$ \\ $\overline{\mathcal{A}}$, closure of $\mathcal{A}, 7$ \\ $\mathfrak{A}$, 个-action on DK, 15 \\ $A^{0}$, unit component of a Lie group $A, 29$ \\ $\mathrm{Ad}$, adjoint action on $\mathrm{K}$ or $\mathfrak{k}, 3$ \\ $\mathrm{B}$, base (set of simple roots) of $\mathrm{R}, 29$ \\ $\mathfrak{B}, \mathrm{T}_{0}$-action on $\mathrm{DK}, 16$ \\ e, open chamber in $t^{*}, 6$ \\ $\overline{\mathrm{C}}$, closure of $\mathcal{C}, 6$ \\ $\mathrm{e}^{\vee}$, dual open chamber in $\mathrm{t}, 7$ \\ $\chi$ bi-invariant three-form, 3 \\ DK, double $\mathrm{K} \times \mathrm{K}, 12$ \\ $\mathrm{G}$, complexification of $\mathrm{K}, 21$ \\ $\Gamma(T)$, exponential lattice, 30 \\ $\Gamma_{\sigma}$, covering group $\left[\mathrm{K}_{\sigma}, \mathrm{K}_{\sigma}\right] \cap \mathrm{Z}\left(\mathrm{K}_{\sigma}\right)^{0}, 9,29$ \\ $\mathrm{H}_{\alpha, n}$, singular hyperplane of $\mathfrak{t}, 28,33$ \\ $\mathrm{i}$, inversion $\mathrm{g} \mapsto \mathrm{g}^{-1}, 4$ \\ impl, implosion, 7 \\ K, compact simply connected Lie group, 3, 7 \\ $K_{\sigma}$, centralizer of a face, 7,29 \\ $\mathrm{K}_{\mathrm{g}}$, centralizer of $\mathrm{g} \in \mathrm{K}, 7$ \\ $\mathfrak{k}_{\sigma}$, Lie algebra of $K_{\sigma}, 29$ \\ L(g), left multiplication by $\mathrm{g}, 3$ \\ M, (quasi-)Hamiltonian K-manifold, 3
}

$\mathrm{M}_{\mathrm{impl}}$, imploded cross-section, 7

$\mathrm{M} / / \mathrm{g} \mathrm{K}$, (quasi-)Hamiltonian quotient, 5

$M_{\sigma}$, cross-section over $\sigma, 7,8$

$\mathrm{N}$, maximal unipotent subgroup of $\mathrm{G}, 21$

$\omega$, (quasi-)symplectic form, 3

$P\left(R^{\vee}\right)$, coweight lattice, 33

$\Phi$, moment map, 3

$\Phi_{\text {impl }}$, imploded moment map, 7

$\Phi_{\sigma}$, moment map on $M_{\sigma}, 8$

$Q\left(R^{\vee}\right)$, coroot lattice, 30

$\mathrm{R}$, root system of $(\mathrm{K}, \mathrm{T}), 28$

$\mathrm{R}_{+}$, positive roots, 31

$R_{\sigma}$, root system of $\left(K_{\sigma}, T\right), 28$

$\mathrm{R}_{\text {min }}$, minimal roots, 29

$\mathrm{R}(\mathrm{g})$, right multiplication by $\mathrm{g}, 3$

$\mathfrak{S}_{\sigma}$, slice at face $\sigma, 7,8,32$

$\sigma$, face of chamber or alcove, 6,28

$\sigma_{\text {prin, }}$ principal face, 6,8

star $\sigma$, star of face $\sigma, 7,8,32$

$\mathrm{T}$, maximal torus of $\mathrm{K}, 6$

$\mathrm{T}_{0}$, extension of $\mathbf{Z} / 2 \mathbf{Z}$ by maximal torus, 16

$\uparrow$, extension of centre by maximal torus, 14

$\theta_{L}, \theta_{R}$, Maurer-Cartan forms, 3

$W$, Weyl group, 7

$w_{0}$, longest Weyl group element, 16

$\mathrm{X}_{\sigma}$, piece $\Phi^{-1}(\exp \sigma) /\left[\mathrm{K}_{\sigma}, \mathrm{K}_{\sigma}\right]$ of $M_{\mathrm{impl}}, 8$

$Z(A)$, centre of a group A, 29

\section{REFERENCES}

[AB83] M. Atiyah and R. Bott, The Yang-Mills equations over Riemann surfaces, Philos. Trans. Roy. Soc. London Ser. A 308 (1983), no. 1505, 523-615. MR 85k:14006

[AMM98] A. Alekseev, A. Malkin, and E. Meinrenken, Lie group valued moment maps, J. Differential Geom. 48 (1998), no. 3, 445-495. MR 99k:58062

[AMW02] A. Alekseev, E. Meinrenken, and C. Woodward, Duistermaat-Heckman measures and moduli spaces of flat bundles over surfaces, Geom. Funct. Anal. 12 (2002), no. 1, 1-31. MR 2003d:53151

[Bor61] A. Borel, Sous-groupes commutatifs et torsion des groupes de Lie compacts connexes, Tôhoku Math. J. (2) 13 (1961), no. 3, 216-240. MR 26 \#5094

[Bou82] N. Bourbaki, Groupes et algèbres de Lie, Éléments de mathématique, Masson, Paris, 1982.

[GJS02] V. Guillemin, L. Jeffrey, and R. Sjamaar, Symplectic implosion, Transform. Groups. 7 (2002), no. 2, 155-184. MR 2003b:53090

[HJ00] J. Hurtubise and L. Jeffrey, Representations with weighted frames and framed parabolic bundles, Canad. J. Math. 52 (2000), 1235-1268.

[HJS] J. Hurtubise, L. Jeffrey, and R. Sjamaar, Moduli of framed parabolic sheaves, in preparation.

[Ler95] E. Lerman, Symplectic cuts, Math. Res. Lett. 2 (1995), no. 3, 247-258. MR 96f:58062

[LMTW98] E. Lerman, E. Meinrenken, S. Tolman, and C. Woodward, Nonabelian convexity by symplectic cuts, Topology 37 (1998), no. 2, 245-259. MR 99a:58069 
[LT97] E. Lerman and S. Tolman, Hamiltonian torus actions on symplectic orbifolds and toric varieties, Trans. Amer. Math. Soc. 349 (1997), no. 10, 4201-4230. MR 98a:57043

[McD88] D. McDuff, The moment map for circle actions on symplectic manifolds, J. Geom. Phys. 5 (1988), no. 2, 149-160. MR 91c:58042

[MS80] V. Mehta and C. Seshadri, Moduli of vector bundles on curves with parabolic structures, Math. Ann. 248 (1980), no. 3, 205-239. MR 81i:14010

[SL91] R. Sjamaar and E. Lerman, Stratified symplectic spaces and reduction, Ann. of Math. (2) 134 (1991), no. 2, 375-422. MR 92g:58036

E-mail address: hurtubis@math.mcgill.ca

Department of Mathematics, McGill University, Montréal, QC H3A 2K6, AND Centre De Recherches Mathématiques, Université de Montréal, Montréal, QC H3P 3J7, CANAdA

E-mail address: jeffrey@math.toronto.edu

Department of MATHEMATICS, UniVERsity of TORONTO, TORONTO, ONTARIO M5S 3G3, CANADA

E-mail address: sjamaar@math. cornell .edu

Department of Mathematics, CORnell UniVersity, IthaCA, NeW York 14853-7901, USA 